NBSIR 76.837

FILE COPY

DO NOT REMONE

\title{
CHARACTERIZATION OF \\ A SUPERCONDUCTING COIL COMPOSITE AND ITS COMPONENTS
}

A F. Clark

V'F Weston

VD. Arp

$J G$ Hust

R.J Trapanı

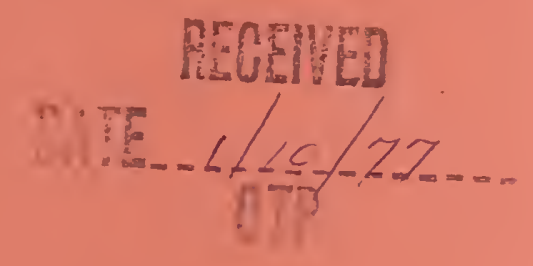

Cryogenics Division

Institute for Basic Standards

National Bureau of Standards

Boulder, Colorado 80302

July 1976

INTERIM REPORT

Prepared for:

Annapolıs Laboratory

Naval Ship Research and Development Center

Annapolis, Maryland 21402 



\section{NBSIR $76-837$}

\section{CHARACTERIZATION OF}

\section{A SUPERCONDUCTING COIL COMPOSITE AND ITS COMPONENTS}

A.F. Clark

W.F. Weston

V.D. Arp

J.G. Hust

R.J. Trapani

Cryogenics Division

Institute for Basic Standards

National Bureau of Standards

Boulder, Colorado 80302

July 1976

INTERIM REPORT

Prepared for:

Ann apolis Laboratory

Naval Ship Research and Development Center

Annapolis, Maryland 21402

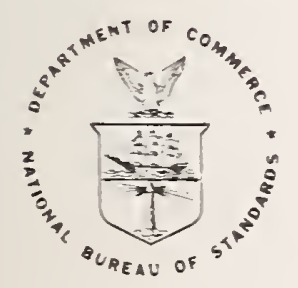

U.S. DEPARTMENT OF COMMERCE, Elliot L. Richardson, Secretary Dr. Betsy Ancker-Johnson, Assistant Secretary for Science and Technology 
List of Tables

List of Figures

Abstract

1. Introduction

2. Mechanical Properties . . . . . . . . . . . . . . . . . . . . . 2

2.1 Young's Moduli and Poisson's Ratio . . . . . . . . . . . . . . . . . 6

2.2 Estimate of Ultimate Strengths . . . . . . . . . . . . . . . . . . 6

2.3 Shear Moduli . . . . . . . . . . . . . . . . . . . . . 14

2.4 Compressibility of Glass Cloth . . . . . . . . . . . . . . . . . 24

References . . . . . . . . . . . . . . . . . . . . . . 28

3. Thermal Properties . . . . . . . . . . . . . . . . . . . . . . 29

3.1 Thermal Conductivity of the Coil Composite . . . . . . . . . . . . . 29

3.2 Thermal Expansion of the Superconducting Wire . . . . . . . . . . . . . 37

3.3 Specific Heat of the Coil Composite and Fiberglass Epoxy . . . . . . . . 44

References . . . . . . . . . . . . . . . . . . . . . 52

4. Elastic Properties by W. F. Weston . . . . . . . . . . . . . . . . . 53

4.1 Introduction . . . . . . . . . . . . . . . . . . . . . . 53

4.2 Experimental Procedure . . . . . . . . . . . . . . . . . . . 55

4.3 Results . . . . . . . . . . . . . . . . . . . . . . 60

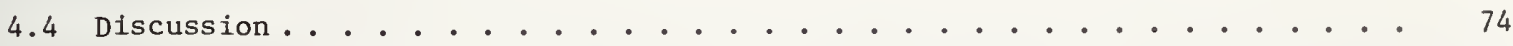

4.5 Conclusions . . . . . . . . . . . . . . . . . . . . . . 79

References . . . . . . . . . . . . . . . . . . . . 80

5. Stress Analysis by V. D. Arp . . . . . . . . . . . . . . . . . . . . 82

5.1 Introduction . . . . . . . . . . . . . . . . . . . . . . 82

5.2 Consultation . . . . . . . . . . . . . . . . . . . . . . 82

5.3 Transversely Isotropic Material Parameters in a Cylindrical Winding . . . 83

5.4 Solution for Stress Distributions in the Absence of Internal Stress . . . 85

5.5 Internal Stress Due to a Uniform Temperature Change . . . . . . . . . . . 86

5.6 Stresses Due to Winding Tension . . . . . . . . . . . . . . . . . . 88

5.7 Epoxy Impregnation of the Coil and Bobbin Removal . . . . . . . . . . . 99

5.8 Magnetic Forces . . . . . . . . . . . . . . . . . . . . . 100 
5.9 9 Puge

5.9 Sumary of Numerical Results . . . . . . . . . . . . . 105

5.10 Conclusions and Recommendations . . . . . . . . . . . . . 118

5.11 Nomenclature . . . . . . . . . . . . . . . . . 123

5.12 Computer Program . . . . . . . . . . . . . . . . 124

References .............................. 129

6. Discussion . . . . . . . . . . . . . . . . . 130

6.1 Comparison with Predicted Values . . . . . . . . . . . . 130

6.2 Preliminary Coll Values . . . . . . . . . . . . . . . . 131

6.3 The Effects of Anisotropy and Temperature . . . . . . . . . . . 134

References ..................... . . 134 


\section{LIST OF TABLES}

1-1. Characterization of superconducting coil composite.

1-2. Considerations of superconducting coil stress analysis for NSRDC.

2-1. Young's moduli and Poisson's ratios for superconducting coil materials.

2-2. Shear moduli for superconducting coil composite.

3-1. Thermal conductivity of longitudinal and transverse sections of superconducting coil composite.

3-2. Thermal expansion of copper-niobium titanium superconducting wire.

3-3. Specific heat of $\mathrm{NbT1/Cu-fiberglass} \mathrm{eopxy} \mathrm{coil} \mathrm{composite} \mathrm{in} \mathrm{temperature} \mathrm{range} 4.0$ to 27.2 (run No. 1).

3-4. Specific heat of $\mathrm{NbTi/Cu-fiberglass} \mathrm{epoxy} \mathrm{coil} \mathrm{composite} \mathrm{in} \mathrm{temperature} \mathrm{range} 4.5$ to $22.2 \mathrm{~K}$ (run No. 2).

3-5. Specific heat of fiberglass-epoxy composite.

3-6. Low-temperature specific heat coefficients for fiberglass-epoxy composite.

3-7. Low-temperature specific heat of fiberglass-composite at specific temperatures.

4-1. Dimensions and orientations of specimens.

4-2. Experiments performed on the superconducting coil composite.

4-3. Parameters in equation 4-7.

4-4. Elastic compliances of the superconducting coil composite at selected temperatures $\left(10^{-10} \mathrm{~m}^{2} / \mathrm{N}\right)$.

4-5. Elastic stiffnesses of the superconducting coil composite at selected temperatures $\left(10^{10} \mathrm{~N} / \mathrm{m}^{2}\right)$.

4-6. Comparison of static and acoustic measurements of elastic constants.

6-1. Comparison of calculated and measured values of coil composite properties. 
1-1. Superconducting coil composite characterization program schematic.

2-1. Schematic of solenoid coll showing construction and coordinate axes.

2-2. Cross-section of the superconducting coil tested.

2-3. Young's modulus versus temperature for superconducting coil materials.

2-4. Polsson's ratio versus temperature for superconducting coll materials.

2-5. Fallure of ultimate strength test specimens.

2-6. Schematic of shear modulus apparatus.

2-7. Schematic of shear displacement measurement system (optical lever arm).

2-8. Shear modulus specimens.

2-9. Shear modulus apparatus calibration data.

2-10. Static shear modulus of coil composite.

2-11. Glass cloth compressibility test apparatus.

2-12. Compressibility of the fiberglass cloth as a function of applied stress.

3-1. Coil composite thermal conductivity specimens.

3-2. Thermal conductivity fixed temperature point apparatus.

3-3. Thermal conductivity of coll composite materials. (Interpolated curves are based on the temperature dependence of similar materials).

3-4. Dilatometer schematic.

3-5. Superconducting wire thermal expansion specimen.

3-6. Comparison of measured and predicted dilatometer calibration curves.

3-7. The thermal contraction of Cu-NbTi wire compared to niobium, titanium, copper, and the longitudinal coll composite.

3-8. Specific heat of the surerconducting coil composite and the fiberglass epoxy at low temperatures.

3-9. Specific heat of the coil composite at the critical temperature.

4-1. Resonant plezoelectric oscillator apparatus.

4-2. Young's modulus of the superconducting coll composite, $\theta=90^{\circ}$.

4-3. Young's modulus of the superconducting $\operatorname{coll}$ composite, $\theta=0^{\circ}$.

4-4. Shear modulus of the superconducting $\operatorname{coll}$ composite, $\theta=0^{\circ}$.

4-5. Shear modulus of the superconducting $\operatorname{col} 1$ composite, $\theta=90^{\circ}$.

4-6. Young's modulus of the superconducting $\operatorname{col} 1$ composite, $\theta=60^{\circ}$.

4-7. Shear modulus of the superconducting $\operatorname{coll}$ composite, $\theta=60^{\circ}$.

4-8. Shear modulus $\mathrm{G}_{23}$ of the superconducting coil composite. 
4-9. Young's modulus of epoxy-fiberglass, $\theta=0^{\circ}$.

4-10. Shear modulus of epoxy-fiberglass, $\theta=0^{\circ}$.

5-1. Radial and circumferential stresses developed by cooldown of a representative magnet coil from $338 \mathrm{~K}$ to $4 \mathrm{~K}$, calculated from equations $5-25$ and 5-26.

5-2. Circumferential stress developed at the inner radius due to a uniform temperature change, normalized by the parameter $L \Delta \alpha \Delta T$ (eq. 5-26), as a function of coll thickness, for several values of the mechanical anisotropy parameter $K$ (eq. 5-17).

5-3. Circumferential stress developed at the outer radius due to a uniform temperature change, normalized by the parameter $L \Delta \alpha \Delta T$ (eq. 5-26), as a function of coil thickness, for several values of the mechanical anisotropy parameter K (eq. 5-17).

5-4. Maximum radial stress due to a uniform temperature change, normalized by the parameter $L \Delta a \Delta T$ (eq. 5-26), as a function of the coil thickness, for several values of the mechanical anisotropy parameter $\mathrm{K}$ (eq. 5-17).

5-5. Radial and circumferential stress calculated from equations 5-42, 43 for a particular coil wound at constant strain of 0.001 , equivalent in this example to a tensile stress of $7.6 \mathrm{kN} / \mathrm{cm}^{2}$, shown as the dashed line. Because of the very low value of $E_{r}$, strain relaxation of up to $55 \%$ occurs within the winding.

5-6. Raidal and circumferential stresses for a particular coil as wound, and after epoxy impregnation and removal of the bobbin on which the coil was wound.

5-7. Radial and circumferential stresses for a particular coil when it generates a field of 3 Tesla at the coil center. As discussed in the text, these results are quite insensitive to reasonable variations in coil material parameters.

5-8. Circumferential stresses in a particular coil after epoxy impregnation and bobbin removal, for four different programed winding tensions, equivalent to the four dashed curves.

5-9. Radial stresses after epoxy impregnation and bobbin removal, for the four different programed winding tensions illustrated in Figure 5-8. Note the change from radial tension in curve A to radial compression in the other three curves.

5-10. Circumferential stress in a particular coil after epoxy impregnation and bobbin removal, for four different values of the radial modulus $T$ (eq. 5-8) prior to epoxy impregnation. 
5-11. The circumferential stress for a particular coil and programed winding strain calculated for four different radial moduli before epoxy impregnation, $\mathrm{E}_{\mathrm{r}}$, using the linear theory in this report (solid 1ines), and using iterative techniques and a non-linear modulus $\mathrm{E}_{\mathrm{r}}$ (dashed line, data calculated by W. Young).

5-12. The radial stress for a particular coll and programmed winding strain calculated for four different radial moduli before epoxy impregnation $E_{r}$, using the linear theory in this report (solid 1ines), and using iterative techniques and non-1inear modulus $\mathrm{E}_{\mathrm{r}}$ (dashed line, data calculated by $\mathrm{W}$. Young).

5-13. The change in circumferential stress for a particular coll as the bobbin is removed, calculated for four different radial moduli before epoxy impregnation, ${ }_{r}$, using the linear theory in this report (solid lines) and using iterative techniques and a non-linear modulus $E_{r}$ (dashed line, data calculated by W. Young).

5-14. The circumferential stress for a particular coll after epoxy impregnation and bobbin remova1, calculated for three different values of $\alpha(=1 . d$. of the bobbin $\div$ o.d. of the bobbin).

5-15. The circumferential stress after epoxy impregnation and bobbin removal, for a given set of material properties, but for four different coll thickness specified by the ratio (outside coll radius $\div$ inside coll radius).

5-16. Cumulative circumferential stresses for a particular coll, calculated for a winding tension which decreases linearly from inside to outside radius (solid lines) and for a winding tension which increases parabolically from the minimum at the inner radius to a maximum in mid-winding and then decreases slightly (dashed lines). The maximum and minimum winding strains are the same in both cases. The dashed 1 ine (4) exhibits a significant reduction in maximum operating stress compared to the solid line (4).

5-17. Cumulative radial stresses for a particular coil, calculated for a winding tension which decreases linearly from inside to outside radius (solid lines) and for a winding tension which increases parabolically from the minimum at the inner radius to a maximum in mid-winding and then decreases slightly (dashed lines). The maximum and minimum winding sirains are the same in both cases. The dashed line (4) exhibits a significant reduction in maximum operating stress compared to the solid 1ine (4). 
A. F. Clark, W. F. Weston, V. D. Arp, J. G. Hust, and R. J. Trapani

\section{ABSTRACT}

The physical properties of a superconducting coil composite and its components are studied in order to accurately predict the coil behavior under a variety of operating conditions. This second interim report includes data on the Young's moduli, the Poisson's ratios, the shear moduli, and the thermal conductivity of the coil composite as well as the compressive modulus of the fiberglass cloth and the thermal expansion of the superconducting wire. In addition, the development of an acoustic technique for the determination of the elastic moduli of the coil composite and the stress analysis of transversely isotropic magnet coils are reported.

Key Words: Fiberglass cloth, mechanical properties, superconducting coil composite, superconducting wire, stress analysis, thermal properties.

\section{INTRODUCTION}

The heart and essential uniqueness of any superconducting motor or generator is the superconducting coil used as a magnetic field source. This coll is subject to a wide range of therma1, mechanical, and magnetic environments and proper understanding of the coil behavior in these environments is necessary to assure not only desired operating behavior but long-term reliability as well. The objective of this research effort has been to determine the physical properties of a superconducting coil composite and its components in order to accurately predict the coil behavior under a variety of conditions.* This second interim report supplements the first ${ }^{1}$ and reports the Young's moduli, the Poisson's ratios, the shear moduli, the thermal conductivity and specific heat of the coil composite as well as the compressive modulus of the fiberglass cloth and the thermal expansion of the superconducting 
wire. In addition, the development of an acoustic technique by one of the authors (W.F.W.) for the determination of the elastic moduli of the coil composite is reported In Section 4 , and the stress analysis by another (V.D.A.) of transversely isotropic magnet coils is reported in Section 5.

A pictorial representation of the general program is shown in Fig. 1-1. The measured properties of the individual components can be related by micromechanical analysis to the measured properties of the coil composite as a whole. This not only provides the composite properties for design application and a check on the micromechanical analysis, but also permits selected modification of the composite by an educated choice of its components. With macromechanics the anisotropic coil properties can be used to develop a stress analysis to predict coil stresses due to the variety of initial and operating conditions such as winding, potting, and cooldown of the coil and magnetic and thermal excursions of the system. Instrumentation of an actual coil will provide a check on the applicability of the stress analysis method and permit selection of coil properties necessary for desirable coil behavior.

The properties measurement program is outlined in Table 1-1 which shows what has been measured and reported in the first report ${ }^{1}$, what is reported here, and what is still to be measured. No symbol indicated no intention of measurement either because it's inapplicable or not needed at this time. The Young's moduli and Poisson's ratios in the first report were not complete so they are reported again in this report. Shown in Table 1-2 are the conditions considered in the development of the stress analysis.

To briefly summarize the first report, first the macroscopic description of an anisotropic composite material was derived and then related to individual constituents by a variety of micromechanical predictive schemes. After describing the mechanical properties and thermal expansion measurements, the results are compared to the values predicted and preliminary coll values are given. For this report the new experimental results are presented and are also compared to predicted values. The effects of temperature and the anisotropy are discussed.

\section{MECHANICAL PROPERTIES}

Several more mechanical properties of the coil composite and its components are reported here. They include the shear moduli of the composite, the compressive moduli of the fiberglass cloth and the Young's modulus along the axial direction $\mathrm{E}_{33}$, and the Poisson's ratio associated with this axial measurement, $\nu_{32}{ }^{\circ}$ A convention of labeling was established 
E)

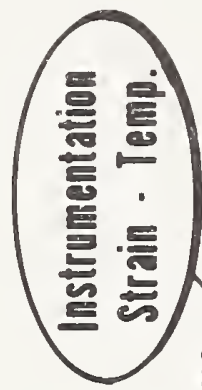

Ex

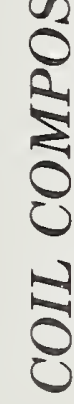

냉
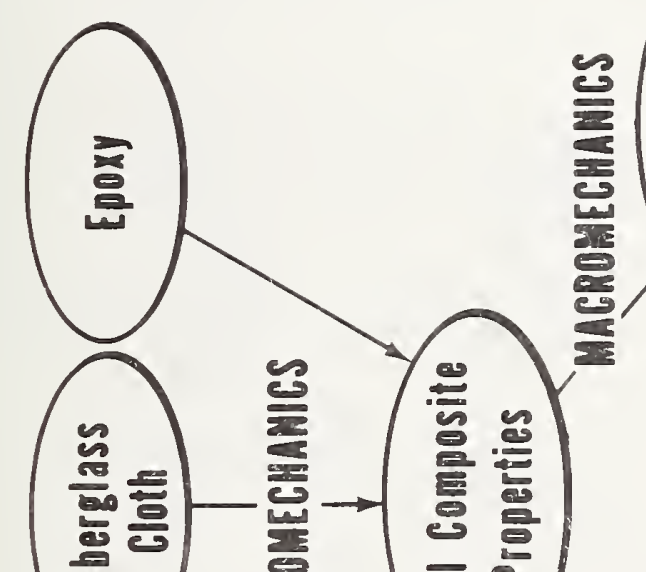

$\rightarrow$ 응

동 6

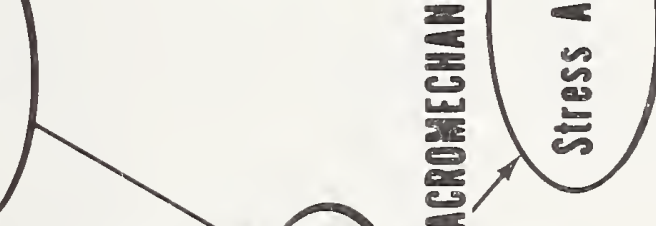




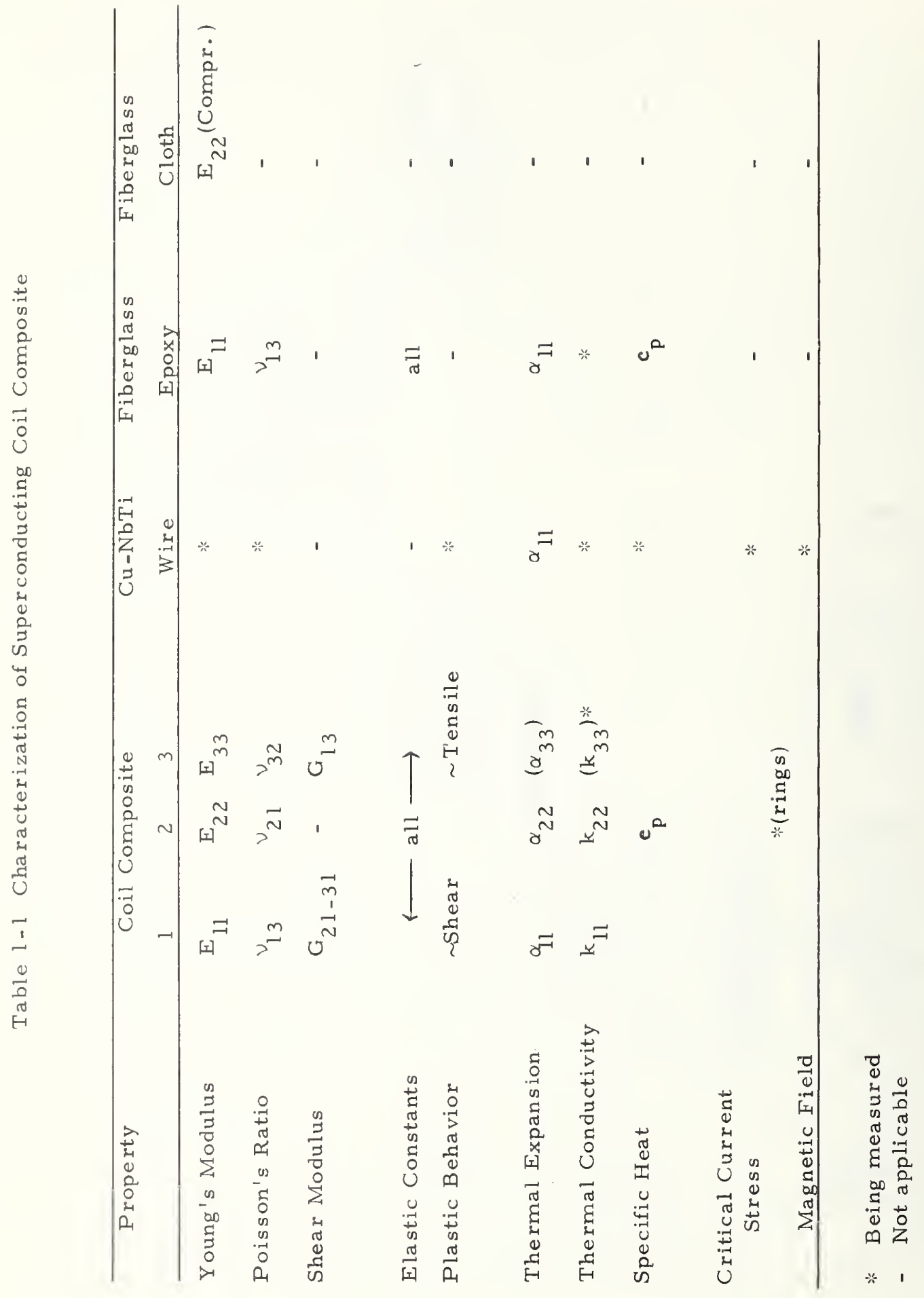


Table 1-2 Considerations of Superconducting Coil Stress Analysis for NSRDC

I. Macromechanical Analysis

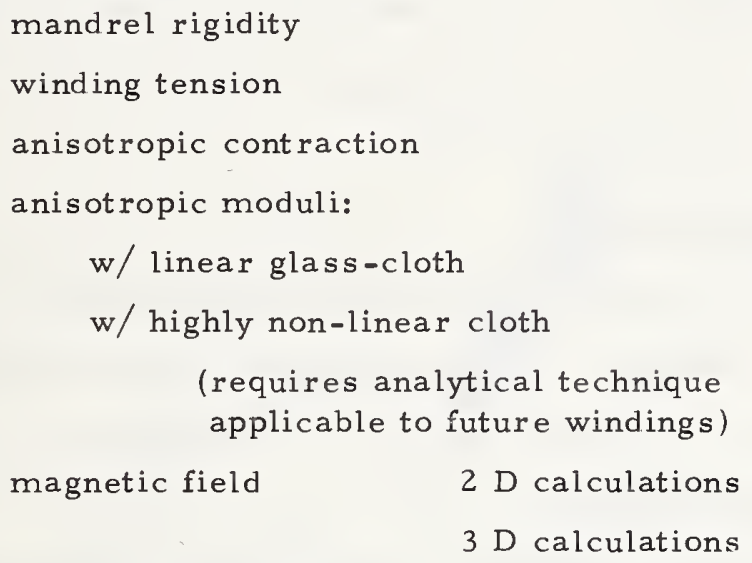

external constraints

II. Micromechanical Analysis

yielding in epoxy around each wire thermal cycling or fatigue

III. Transient Thermal Stresses

cooldown

magnet quench 
in the first report ${ }^{1}$ with (1) being the longitudinal axis along the wires of the solenoid, (2) perpendicular to the wires in the radial direction of the solenoid, and (3) perpendicular to the wires in the axial direction of the solenoid. This is shown schematically in Figure 2-1. That the (2) and (3) directions are not identical can be seen in the photographic cross section of Figure 2-2 which shows the gap created between the layers of the nearly rectangular wires created by the wrap of fiberglass cloth between each layer. Thus in the photograph the (3) direction is along this layer of cloth, (2) perpendicular to it, and (1) perpendicular to the page. The measured volume fraction of the wire taken from enlargements of similar micrographs is 0.73 .1

\subsection{Young's Moduli and Poisson's Ratios}

The specimen preparation and experimental arrangement are described in detail in the preceding report. ${ }^{1}$ Briefly, the specimens were machined from actual coil material, strain gauges applied, and measurements performed at the three temperatures, 293,76 and $4 \mathrm{~K}$, in a normal low temperature tensile arrangement.

The only difference for the tests reported here was that the longitudinal and transverse gauges were both monitored at the same time using two bridge networks and an $X-Y-Y$ recorder. This reduced the number of loadings required for each specimen.

Because of the relatively weak fracture properties of the epoxy bond between wires, the load for the axial specimen was limited to $\sim 1.0 \mathrm{MN} / \mathrm{mm}^{2}$ ( $\sim 1000$ psi). The resultant strains were quite small (especially for the Poisson gauge output) and the noise limited resolution yields an estimated uncertainty of about 5\% for the Young's modulus and $12-15 \%$ for the Poisson's ratio. The data for all of the Young's moduli and Poisson's ratios are tabulated in Table 2-1 and shown in Figures 2-3 and 2-4.

The behavior of the axial Young's modulus, $E_{33}$, is, as expected, very similar to that of the radial specimen, $\mathrm{E}_{22}$. It is probably due almost entirely to the epoxy. The Poisson's ratio, $v_{23}$, has a value between the longitudinal $\nu_{3}$, which would be expected to behave more like the Cu-NbTi wire, and the radial $\nu_{21}$, which behaves similar to the fiberglass epoxy. This is as might be expected. The slight increase with decreasing temperature is similar to the Young's moduli.

\subsection{Estimate of Ultimate Strengths}

Specimens for all three principal directions were pulled to failure at $4 \mathrm{~K}$ in an attempt to estimate the ultimate strengths. The radial specimen failed at a very low load 


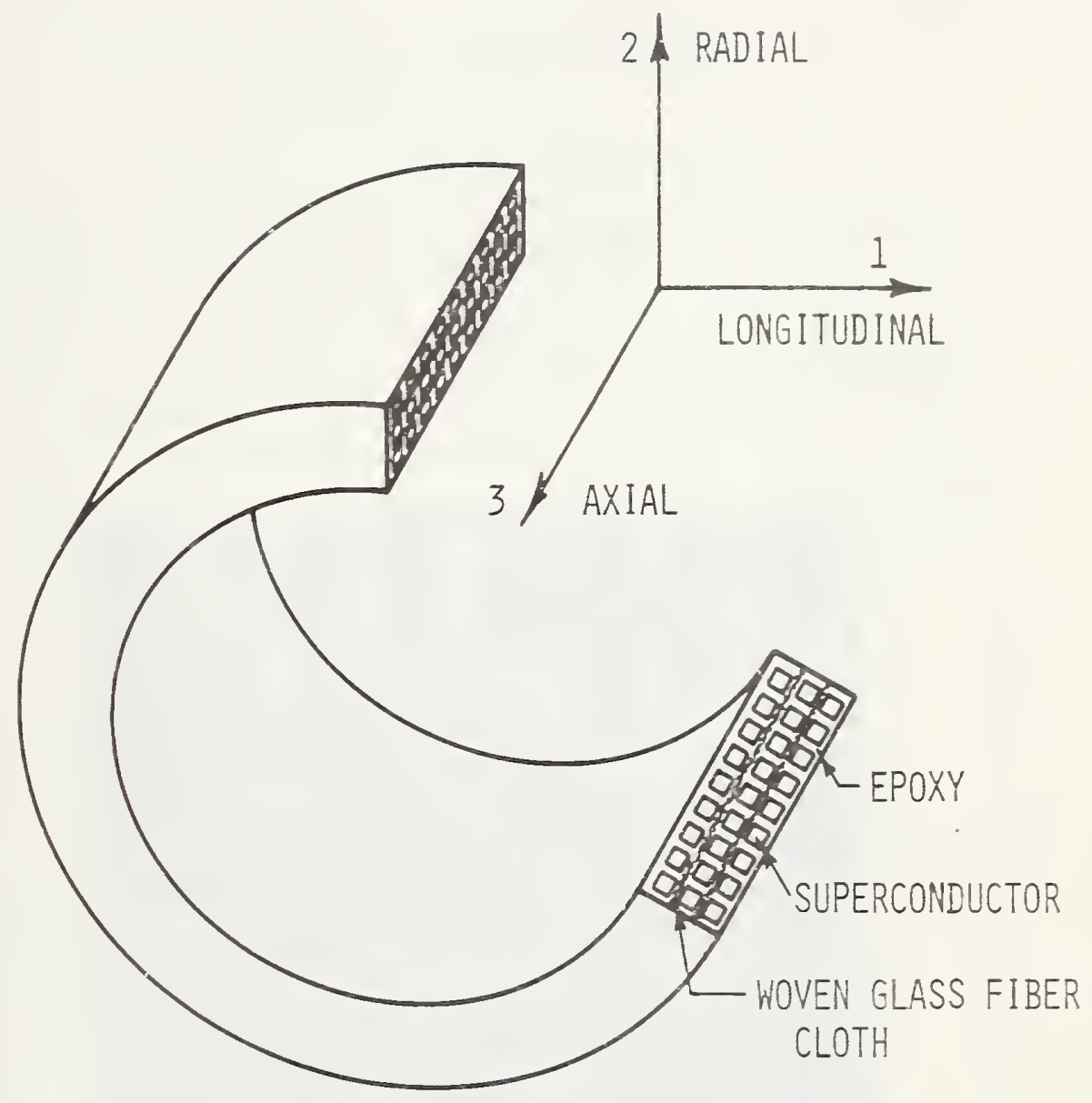

Figure 2-1. Schematic of solenoid coil showing construction and coordinate axes. 


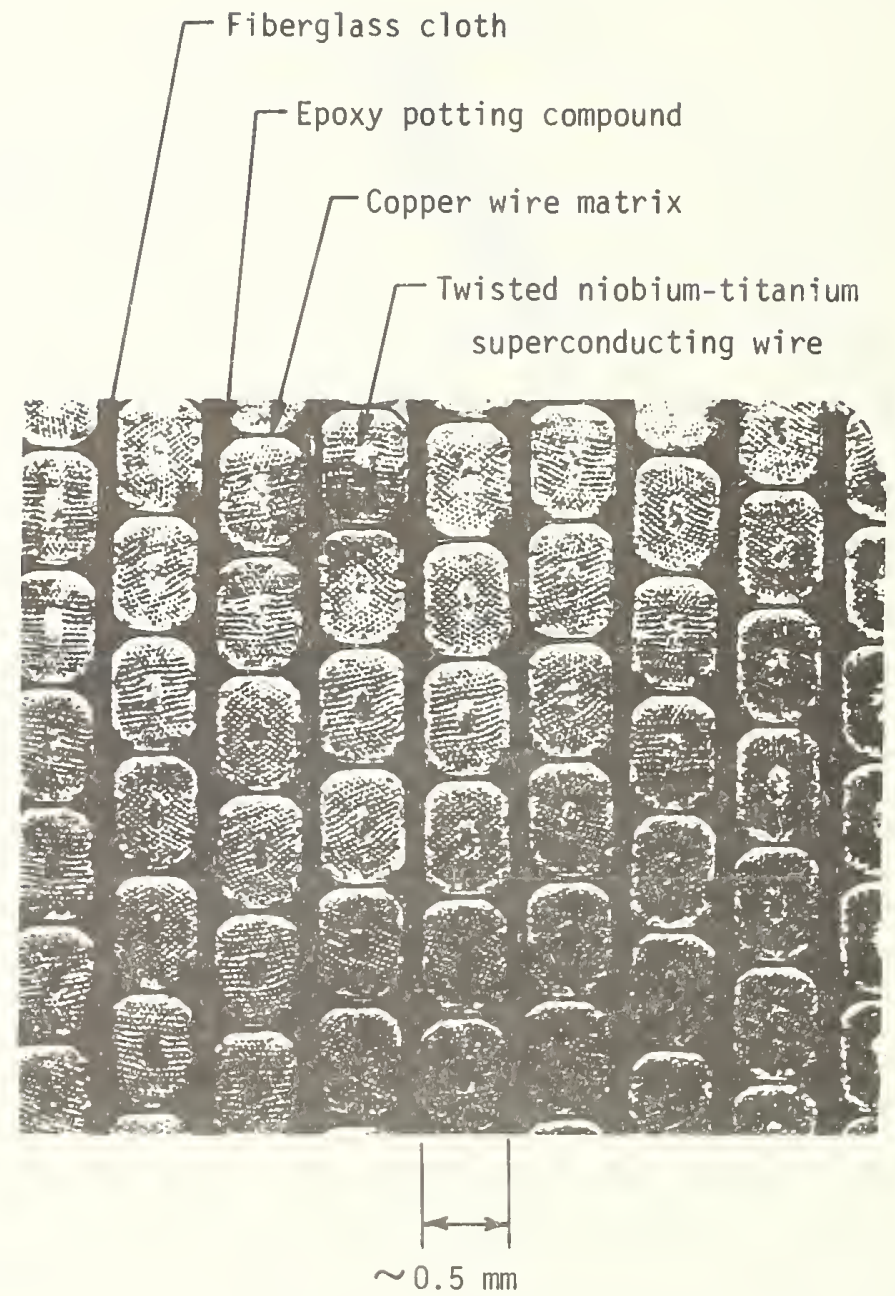

Figure 2-2. Cross-section of the superconducting coll tested. 
Table 2-1 Young's Moduli and Poisson's Ratios for Superconducting Coil Materials

\begin{tabular}{|c|c|c|c|c|c|}
\hline \multirow{2}{*}{\multicolumn{2}{|c|}{ Specimen }} & \multicolumn{4}{|c|}{ YOUNG'S MODULI $10^{4} \mathrm{MN} / \mathrm{m}^{2}\left(10^{6} \mathrm{psi}\right)$} \\
\hline & & & 293 & 77 & 4 \\
\hline Radial & $\# 1$ & $\left(E_{22}\right)$ & $2.82(4.10)$ & $3.82(5.53)$ & $4.15(6.01)$ \\
\hline Radial & $\# 2$ & $\left(E_{22}\right)$ & $2.68(3.89)$ & $3.75(5.43)$ & $4.08(5.91)$ \\
\hline Long. & $\# 1$ & $\left(E_{11}\right)$ & $7.72(11.21)$ & $8.26(11.97)$ & $8.74(12.67)$ \\
\hline Long. & $\# 3$ & $\left(E_{11}\right)$ & $7.39(10.72)$ & $7.89(11.44)$ & $8.31(12.05)$ \\
\hline$F \cdot E$. & $\# 1$ & $\left(E_{11}\right)$ & $2.82(4.10)$ & $3.33(4.83)$ & $3.56(5.16)$ \\
\hline Transv. & $\# 1$ & $\left(E_{33}\right)$ & $2.28(3.3)$ & $3.10(4.5)$ & $3.10(4.5)$ \\
\hline
\end{tabular}

POISSON'S RATIOS

Temperature, Kelvin

\begin{tabular}{lcccc}
\multicolumn{1}{l}{ Specimen } & & 293 & 77 & 4 \\
\hline Radial \#1 & $\left(\nu_{21}\right)$ & 0.146 & 0.202 & 0.196 \\
Radial \#2 & $\left(\nu_{21}\right)$ & 0.199 & 0.248 & 0.256 \\
Long. \#3 & $\left(\nu_{13}\right)$ & 0.333 & 0.352 & 0.352 \\
F.E. $\quad \# 1$ & $\left(\nu_{13}\right)$ & 0.149 & 0.209 & 0.221 \\
Transv. \#1 & $\left(\nu_{32}\right)$ & 0.278 & 0.300 & 0.321 \\
\hline
\end{tabular}




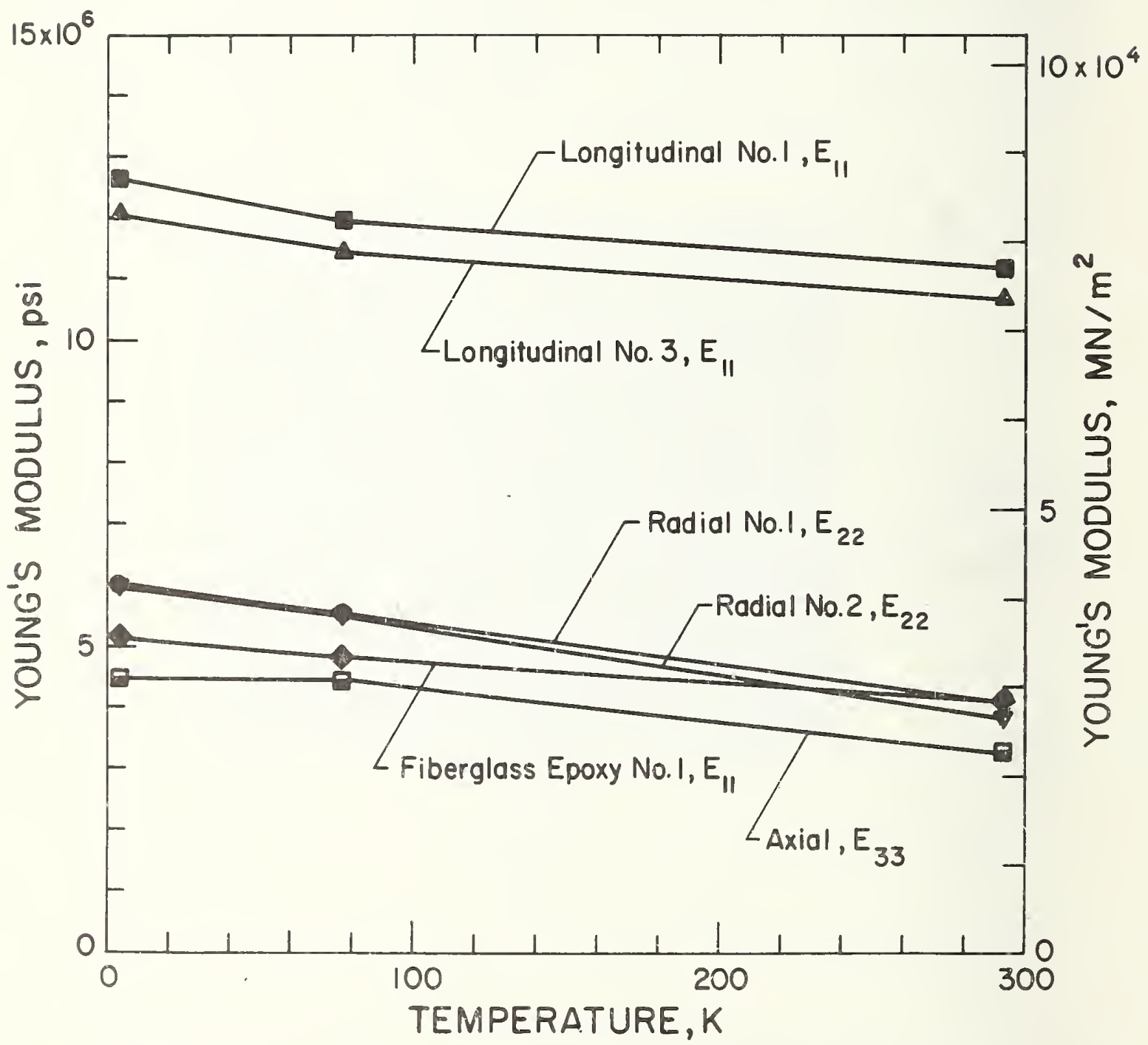

Figure 2-3. Young's modulus versus temperature for superconducting coll materials. 


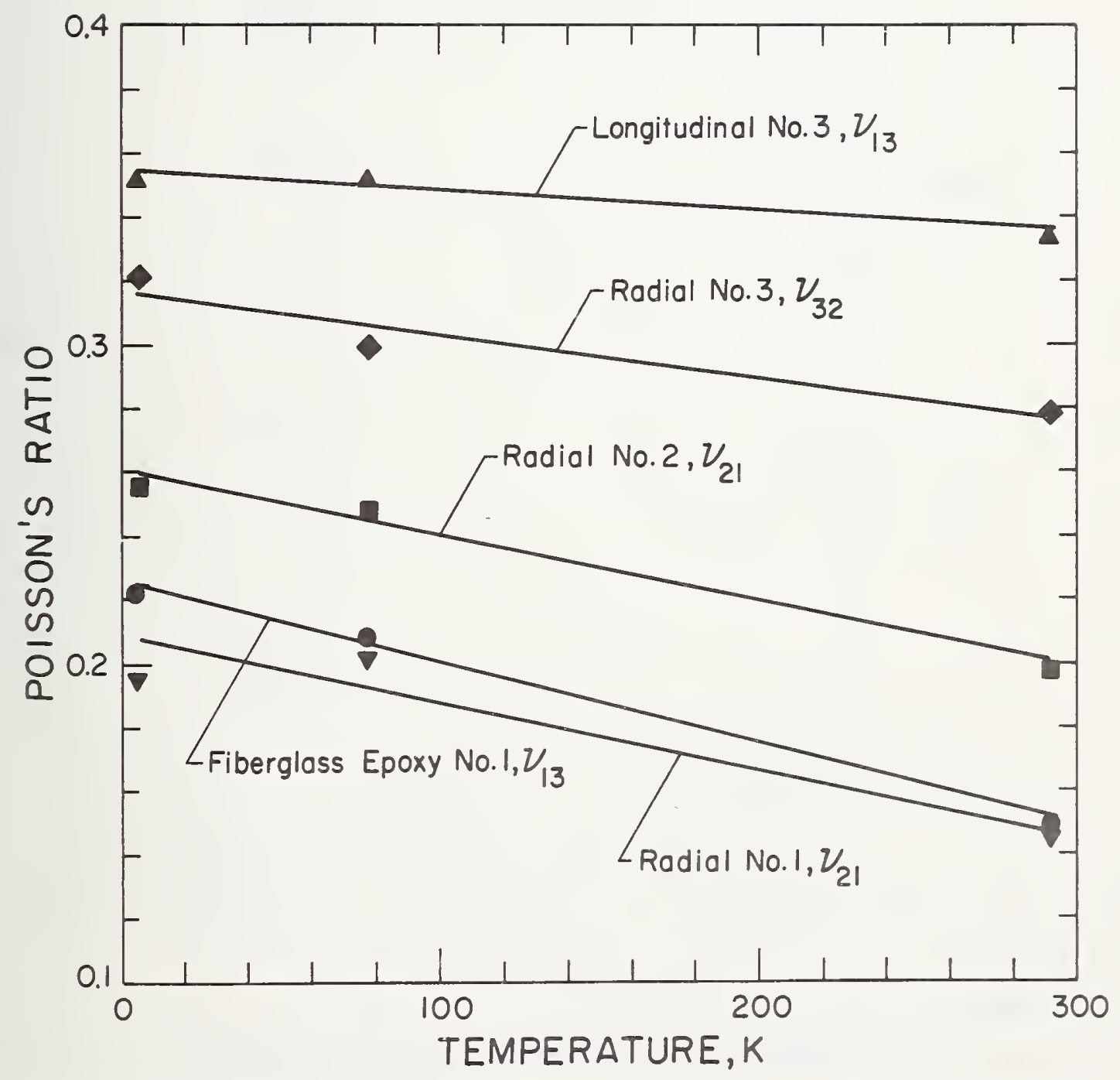

Figure 2-4. Poisson's ratio versus temperature for superconducting coil materials. 
at one of the epoxy joints of its fabrication ${ }^{1}$ and is therefore meaningless. Both the longitudinal and axial specimens failed in the grips, but with measurements of crosssectional areas and estimates of stress concentration factors rough approximations of ultimate strengths can be calculated. The failure modes are shown in Figure 2-5, from which it can be seen that the axial specimen failed in tension and the longitudinal in shear.

One method of estimating ultimate strength in the axial specimen would be to consider the net area at the fallure line, using

$$
A_{N}=A_{G}-A_{C}
$$

where

$$
\begin{aligned}
& A_{N}=\text { net area at fallure line } \\
& { }_{G}=\text { gross cross-sectional area at failure line } \\
& A_{C}=\text { area of circular hole at failure line }
\end{aligned}
$$

and by using

$$
\sigma=P / A_{N}
$$

where

$$
\begin{aligned}
& \sigma=\text { apparent stress } \\
& \mathrm{P}=\text { measured output of load cell }
\end{aligned}
$$

and also

$$
\sigma_{\mathrm{U}}=\mathrm{k} \sigma
$$

where

$$
\begin{aligned}
& \sigma_{U}=\text { estimated ultimate strength } \\
& k=\text { stress concentration factor. }
\end{aligned}
$$

A value of 3 for the stress concentration factor is used. ${ }^{2}$ The approximate ultinate strength for the axial specimen at $4 \mathrm{~K}$, as estimated without a stress concentration factor, is $57 \mathrm{MN} / \mathrm{m}^{2}$ (8300 psi). Calculated with a stress concentration factor of 3 , an estimate for ultimate strength is $171 \mathrm{MN} / \mathrm{m}^{2}(24,800 \mathrm{psi})$.

The failure in the longitudinal specimen was due to shear batween wires of the composite perpendicular to the glass cloth. An estimate of ultimate shear strength can be made using the equation:

$$
\sigma_{S}=P / A
$$



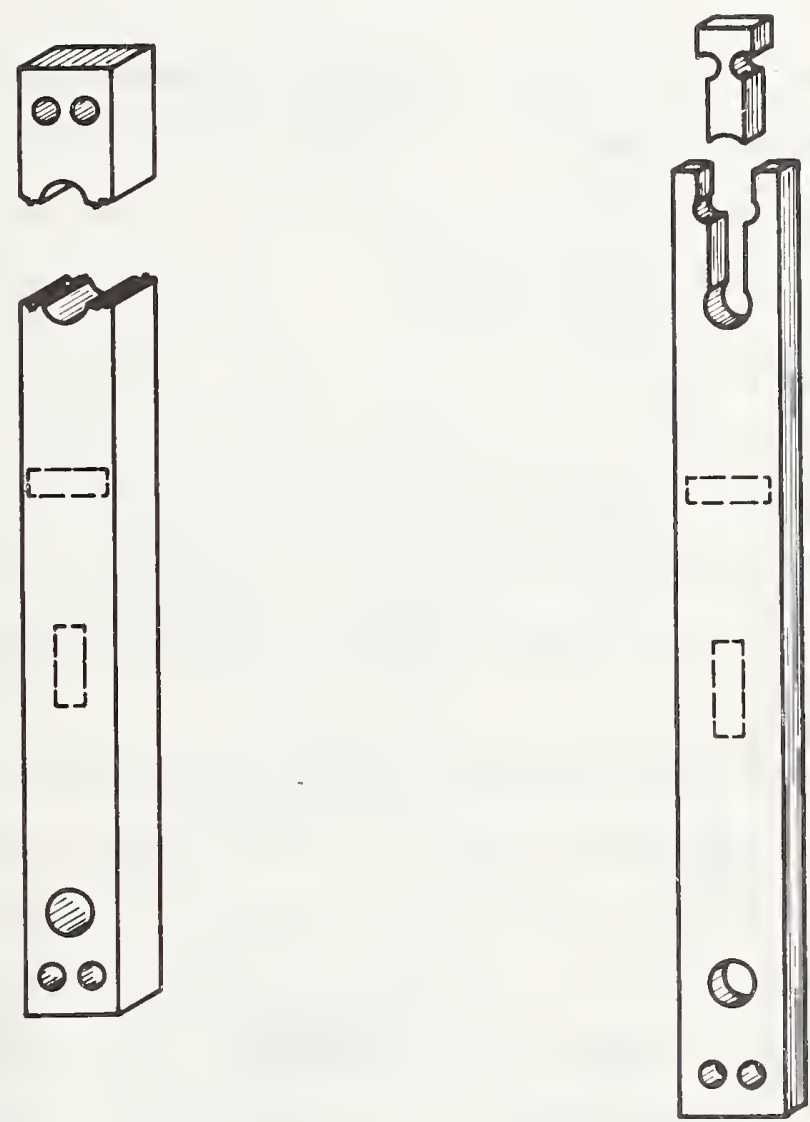

AXIAL

LONGITUDINAL

Figure 2-5. Failure of ultimate strength test specimens. 
where

$$
\begin{aligned}
& \sigma_{S}=\text { ultimate shear strength } \\
& P=\text { ultimate load } \\
& A=\text { area of shear failure. }
\end{aligned}
$$

Using this equation, estimated ultimate shear strength is about $107 \mathrm{MN} / \mathrm{m}^{2}(15,500 \mathrm{psi})$ at $4 \mathrm{~K}$. No stress concentration factor has been used. These estimates should be considered just that and nothing more.

\subsection{Shear Moduli}

The shear moduli were measured using a version of the apparatus developed by Mikesell and McClintock. ${ }^{3}$ The apparatus consists of two concentric stainless steel cylinders in which the specimen is secured and is schematically shown in Figure 2-6. The inner cylinder moves in a teflon bearing attached to a brass plate, while the outer cylinder is securely attached to the plate. The plate rests on a glass dewar with the cylinders suspended in the cryogen. The torque is applied to the specimen by slotted weights on a pan which, via a pulley and butterfly system, is transferred to the inner cylinder and specimen. The torsion of the specimen is measured by observing the displacement of an optical image of a laser beam formed on a distant translucent scale from a mirror attached to the top of the inner cylinder.

Modifications to the original apparatus included a strip-silvered outer glass dewar which allowed visual monitoring of liquid levels and also provided a liquid nitrogen shield. The original optical lever arm was modified to allow the use of a laser beam image. A low power helium-neon laser was used. Alignment of the laser optical arm is accomplished by adjusting the laser to shine on the mirror and then adjusting the mirror so the image falls on the translucent scale (see Figure 2-7). Due to the various sizes of the specimens tested, in some cases a heavier set of weights was used. These heavier weights caused a slight torsion in the inner cylinder itself, however the effect was calculated to be negligible.

Two specimen geometries were tested. The first type was made by winding layers of wire and cloth around a flat plate. One linear section was then used. This specimen configuration is referred to as "longitudinal". The second type of specimen was manufactured by winding the superconducting wire around a cylindrical mandre1, with a 1 ayer of fiberglass cloth applied between each winding of wire. This type of specimen is basically 


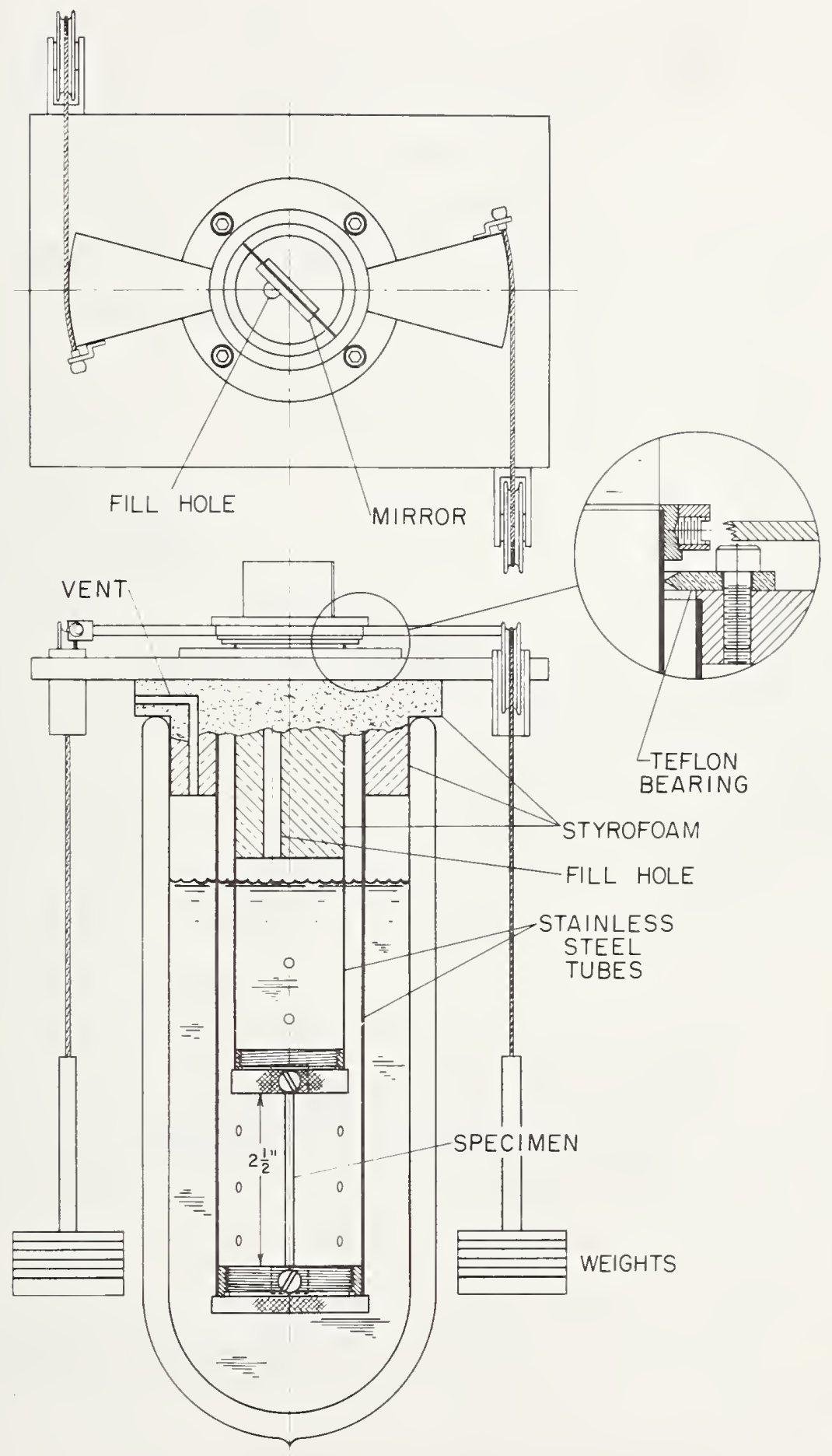

Figure 2-6. Schematic of shear modulus apparatus. 


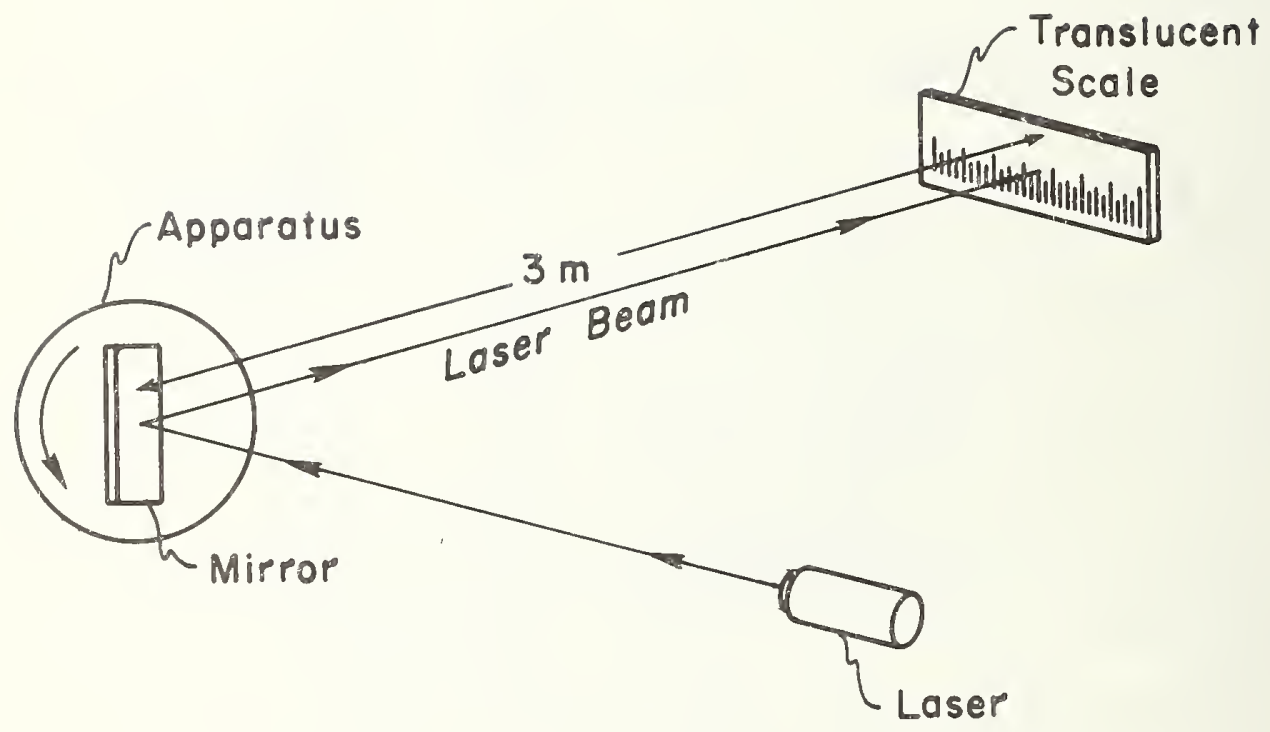

NOTE:

Beam, Front Surface of Mirror, and

Center of Rotation should be Coincident.

Figure 2-7. Schematic of shear displacement measurement system (optical lever arm). 
a very small radius solenoid and is referred to as "cylindrical". After the specimens were wound, they were placed in a potting mold and impregnated with the epoxy. The entire systems were then cured for eight hours at $150^{\circ} \mathrm{F}$.

Materials used for calibration specimens included aluminum 1100 and 17-4PH stainless steel. These specimens were in the cylindrical rod specimen configuration as used in reference 3. All the specimens are pictured in Figure 2-8.

The longitudinal specimen and also the calibration specimens were mounted using the method in 3. The cylindrical specimen required special adaptors as shown in Figure 2-8. The ends of the specimen were notched to fit over a matching pin when inserted into the adaptor. Epoxy was then poured into the dished portion and the specimen was cured. This provided a positive mechanical bond which withstood many low-temperature cycles.

The convention for the notation of the shear moduli is that $G_{i j}$ is for shear with the force in the $i^{\text {th }}$ direction in the $j^{\text {th }}$ plane (the plane perpendicular to $\vec{j}$ ). This convention is also shown in Figure 2-8.

Room temperature runs began with mounting the specimen and then assembling the apparatus. After checking the distance from the mirror to the scale, the optical lever arm was aligned to the translucent scale. Pairs of weights are then successively placed on the weight pans, and the angular displacements of the specimen are noted. Five pairs of weights were applied. To minimize the effects of hysteresis of the specimens and apparatus, the load is increased to a maximum and then back to a minimum (load-unload cycle), with angular displacements measured for both.

The procedure for low-temperature runs is similar to the room temperature procedure except the specimen and apparatus are first cooled with liquid nitrogen. For $4 \mathrm{~K}$ measurements the inner dewar is then purged of liquid nitrogen and filled with liquid helium. After the transfer is completed, the mirror and teflon bearing are ventilated with room temperature air to prevent frost and subsequent binding between the inner cylinder and teflon bearing. The specimen is then put through several load-unload cycles and the angular displacements are measured.

The apparatus was calibrated by measuring the shear moduli of two calibration materials (aluminum 1100 and stainless steel 17-4PH) and comparing the measured values to known values determined previously ${ }^{4}$ and by an acoustic technique. ${ }^{5}$ Figure 2-9 shows the known and measured values plotted versus temperature. It is noted that there is a $3 \%$ and $4.5 \%$ discrepancy 

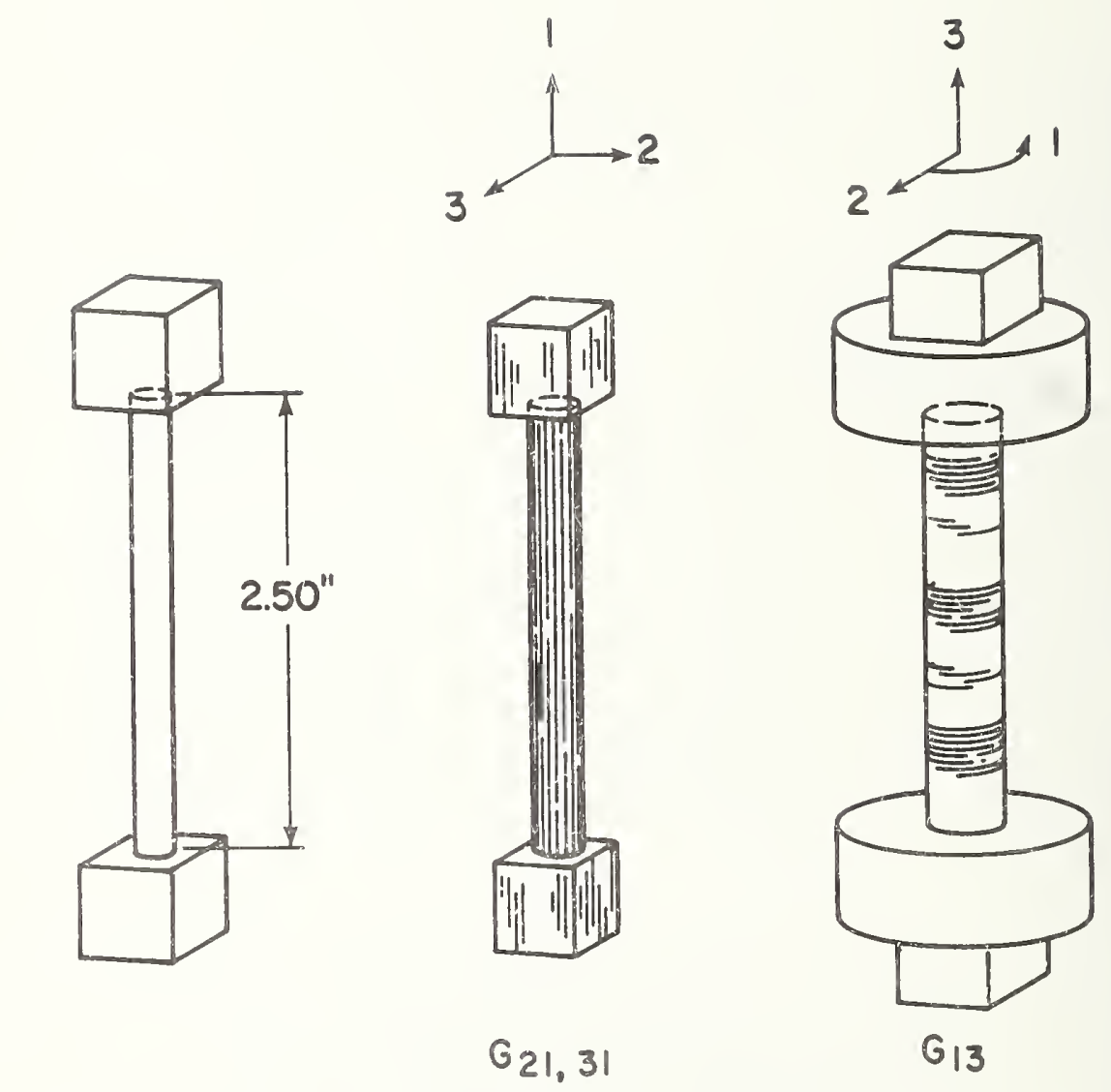

CALIBRATION LONGITUDINAL

CYLINDRICAL

Figure 2-8. Shear modulus specimens. 


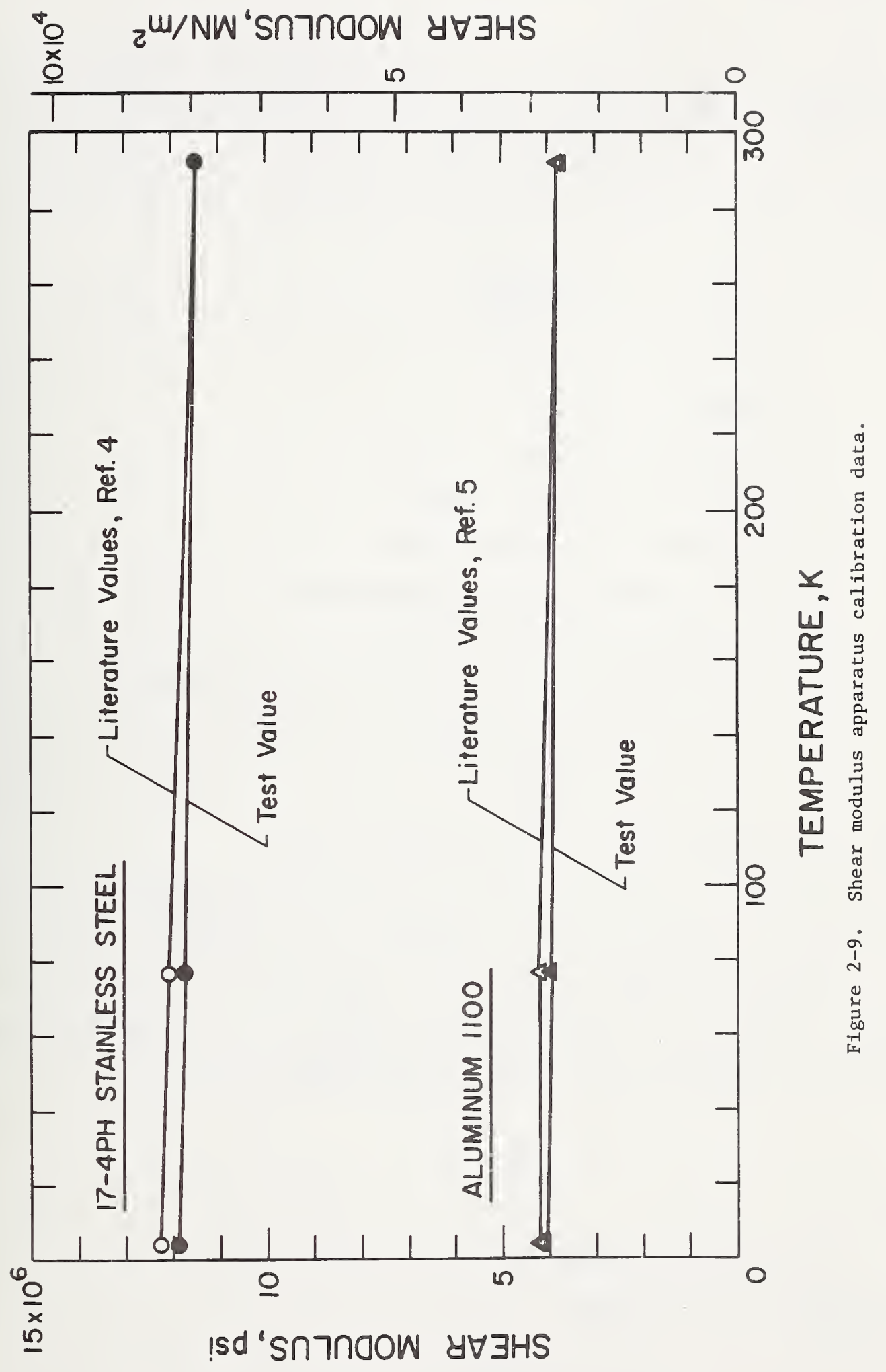


at low temperatures. This inconsistency is still unresolved and the data are presented for the coil material uncorrected.

Experimental data were reduced by first plotting angular displacement vs load and then fitting a line to the data by using a linear least squares program. The first and last data points of each run were not included in the fit so as to minimize the effect of hysteresis. After the slope of the line was determined, the shear modulus was computed from:

where

$$
G=\frac{64 w R l L}{J \frac{\Delta S}{L}}
$$

$$
\begin{aligned}
& \mathrm{w}=\text { the weight on specimen } \\
& \mathrm{R}=\text { the radius arm } \\
& \ell=\text { the effective length of specimen } \\
& \mathrm{L}=\text { the optical lever arm length } \\
& \mathrm{J}=\text { the polar moment of inertia of the specimen } \\
& \Delta S=\text { the deflection of the optical image. }
\end{aligned}
$$

This equation is derived from the basic equation for torsion in a shaft:

$$
\theta=\frac{T l}{J G}
$$

where, $\theta=$ the amount of twist and $\mathrm{T}$ is the applied torque.

In equation(2-6), it can be noted that the specimen length and also the diameter of the specimen will change with the test-temperature. The values were corrected by using a thermal expansion coefficient for the material, which was found by Fowlkes et al. ${ }^{1}$ This correction varied for the different specimen geometries. This correction was significant because the polar moment of inertia for the longitudinal specimen is:

$$
J=\frac{\pi d^{4}}{32}
$$

where,

$$
\mathrm{d}=\text { the specimen diameter }
$$

and for the cylindrical specimen:

$$
J=\frac{\pi\left(d_{0}^{4}-d_{i}^{4}\right)}{32}
$$


where,

$$
\begin{aligned}
& d_{0}=\text { the outer diameter of the specimen } \\
& d_{i}=\text { the inner diameter of the specimen. }
\end{aligned}
$$

Since the specimen dlameter for both cases enters into the calculation to the fourth power, the thermal expansion correction must be carefully applied.

As reported in 3, the most significant errors arise from d and $\Delta S$. Due to irregularities on the surface of the specimens, the diameter was measureable to $2 \times 10^{-3}$ inches, which gives an uncertainty in $\mathrm{d}^{4}$. of about $1 \%$. Using a 3 meter optical lever arm, the maximum standard deviation for a set of five measurements (at each temperature) on the coil material was about $7 \%$.

Average values and the data found are presented in Figure 2-10 and Table 2-2. A size effect was noted on further testing of the cylindrical specimen. A layer of superconducting wire was machined off and the specimen was tested at room-temperature and in liquid nitrogen. An increase in shear modulus of about $20 \%$ and $15 \%$ were noted at $293 \mathrm{~K}$ and $77 \mathrm{~K}$, respectively. This increase can be explained by the fact that a greater volume fraction of wire was present in the machined-down specimen, thus causing an increase in the shear modulus. Shear modulus of the coil material can be predicted by the solution of the mixture laws of composite materials, which are derived from simplified microstructural models. In the previous report Fowlkes et $a 1^{1}$ predicted shear modulus values for the cylindrical specimen at room temperature ranging from $.346 \times 10^{4} \mathrm{MN} / \mathrm{m}^{2}\left(.502 \times 10^{6} \mathrm{psi}\right)$ to $.916 \times 10^{4}$ $\mathbb{M N} / \mathrm{m}^{2}$ ( $1.33 \times 10^{6} \mathrm{psi}$ ), depending on the microstructural model chosen. It is noted that the predicted values bracket the experimental value for this specimen configuration.

The acoustic measurements of the elastic constants were made at about the same time as these static tests, and inftial results indicated that the two techniques were yielding nearly identical results (See Table 4-6). In addition, it was determined that for a transversely isotropic material $G_{13}$ should be approximately equal to $G_{31}$ and $G_{21}$. The difference between the two static tests $(\sim 20 \%)$ is therefore an indication of the effect of curvature and/or the averaging effect in the Iongitudinal specimen of $G_{21}$ and $G_{31}$. Because of these results the static tests were considered a sufficient check on the acoustic technique and were abandoned in favor of the more rapid and accurate acoustic measurements reported in Section 4. The comparisons are more accurately described in Section 4.4. 


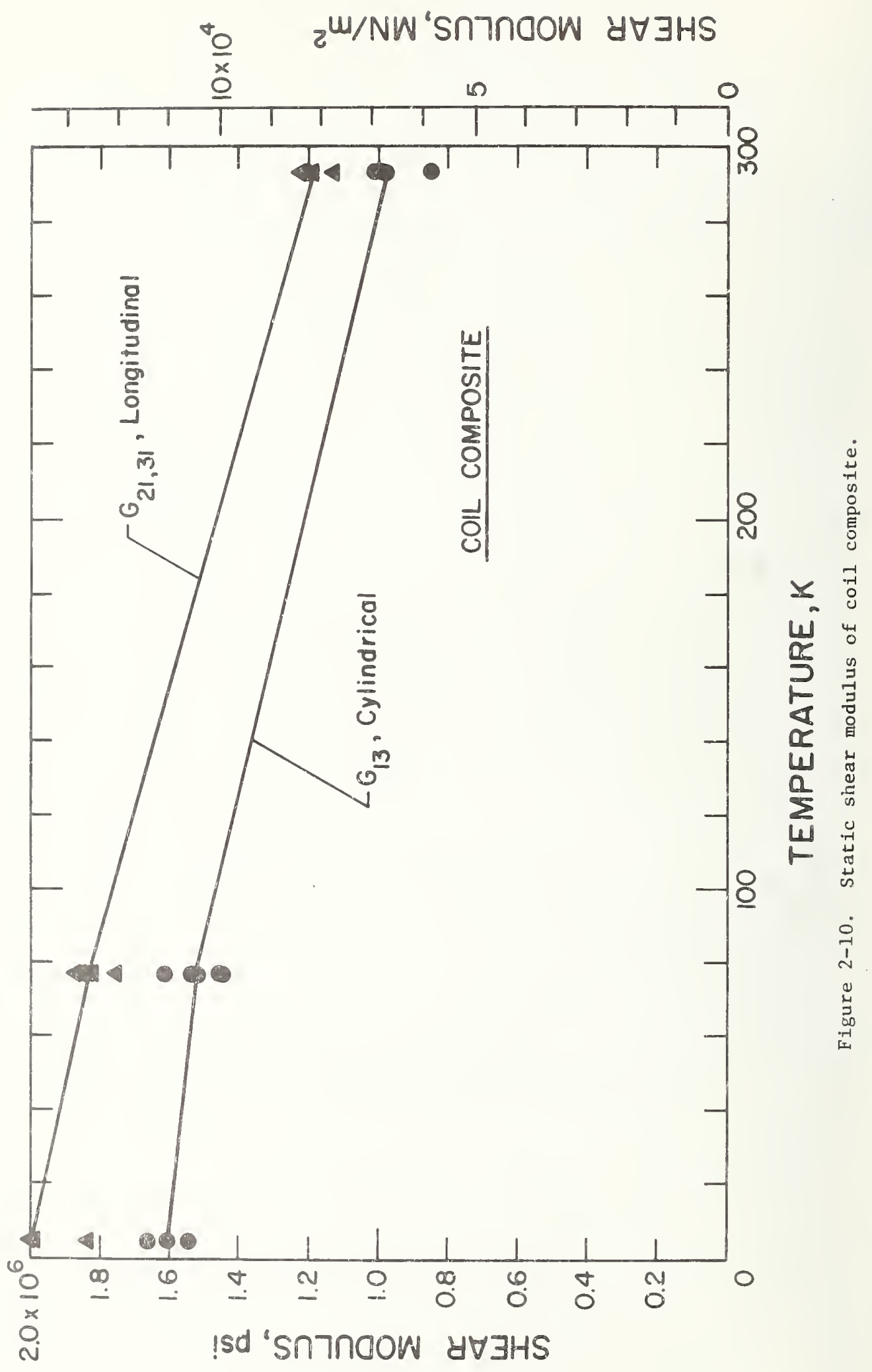


Table 2-2 Shear Moduli for Superconducting Coil Composite

$$
10^{4} \mathrm{MN} / \mathrm{m}^{2}\left(10^{6} \mathrm{psi}\right)
$$

\begin{tabular}{|c|c|c|c|c|}
\hline \multicolumn{5}{|c|}{ Temperature, Kelvin } \\
\hline & & 293 & 77 & 4 \\
\hline Longitudinal & (Ave.) & $0.82(1.19)$ & $1.27(1.84)$ & $1.33(1.93)$ \\
\hline $\mathrm{G}_{21,31}$ & (Data) & $\begin{array}{l}0.82(1.19) \\
0.78(1.13) \\
0.83(1.21) \\
0.84(1.22) \\
0.82(1.19)\end{array}$ & $\begin{array}{l}1.28(1.86) \\
1.21(1.76) \\
1.30(1.88) \\
1.26(1.83) \\
1.28(1.86) \\
1.27(1.84)\end{array}$ & $\begin{array}{l}1.26(1.83) \\
1.38(2.00) \\
1.34(1.95)\end{array}$ \\
\hline Cylindrical & (Ave.) & $0.67(0.975)$ & $1.05(1.52)$ & $1.11(1.61)$ \\
\hline $\mathrm{G}_{13}$ & (Data) & $\begin{array}{l}0.75(1.09) \\
0.68(0.988) \\
0.68(0.979) \\
0.58(0.844)\end{array}$ & $\begin{array}{l}1.05(1.52) \\
1.06(1.54) \\
1.00(1.45) \\
1.12(1.62) \\
1.01(1.46)\end{array}$ & $\begin{array}{l}1.11(1.61) \\
1.07(1.55) \\
1.15(1.67)\end{array}$ \\
\hline
\end{tabular}




\subsection{Compressibility of Glass Cloth}

The compressibility of the glass cloth which separatessucceeding magnet layers is an important factor determining internal magnet stresses. A brief literature survey disclosed no information on this topic for stress levels up to tens of Mega-Newtons per $\mathrm{m}^{2}$, as are relevant for typical coils. Consequently, we assembled and performed the following compressive modulus tests on the glass cloth used by the sponsors and supplied by them.

The coil winding is done at room temperature so only room temperature measurements were made. A test jig shown in Figure 2-11 was assembled for use in our tensile testing machine. Nine layers of glass cloth, seperated one from another by flat brass washers, cumulatively provided enough compression that its magnitude could be measured with reasonable accuracy using clip-on strain gauges which are standard in our laboratory. The brass washers were carefully machined so that the maximum variation in thickness around any given washer was no more than about $0.0010 \mathrm{~cm}$, so as to insure a reasonably uniform loading of the $0.010 \mathrm{~cm}$ thick (nominal) glass cloth. We wereaided in this respect by the extremely low compressive modulus of the cloth at low pressures, such that an initial loading of no more than about $10^{5} \mathrm{~N} / \mathrm{m}^{2}$ is sufficient to cause a compression of $0.001 \mathrm{~cm}$ per layer of cloth. Layers of gasket rubber, totalling about $0.5 \mathrm{~cm}$ thick, are placed on either end of the test $j i g$ (outside of the gauge length) to take up any lack of parallelism which may exist in the elements which apply the compressive loads.

Two sets of washers were used; a large washer, of area $23.67 \mathrm{~cm}^{2}$, for obtaining good resolution at lower stress levels with minimized effects due to any residual force misalignment, and a smal.1 washer, of area $4.434 \mathrm{~cm}^{2}$, for obtaining higher compressive pressures within the constraints imposed by the tenslle testing equipment. Where the respective data with these two washer sizes overlapped, the derived values of $\mathrm{d} \sigma / \mathrm{d} \varepsilon(\sigma)$ agreed within a factor of two, which is fair considering the large scatter and hysteresis which were observed.

Results are plotted in Figure 2-12. Because of the very large hysteresis and nonlinearity in the curves of $\varepsilon(\sigma)$, the logarithmic plots are necessary in order to bring the results into a useable form. Considerable scatter is also seen, especially when the testing machine is reversed under high load, so that $\sigma$ begins to decrease steadily with time. As can be seen, results can be expressed approximately by 


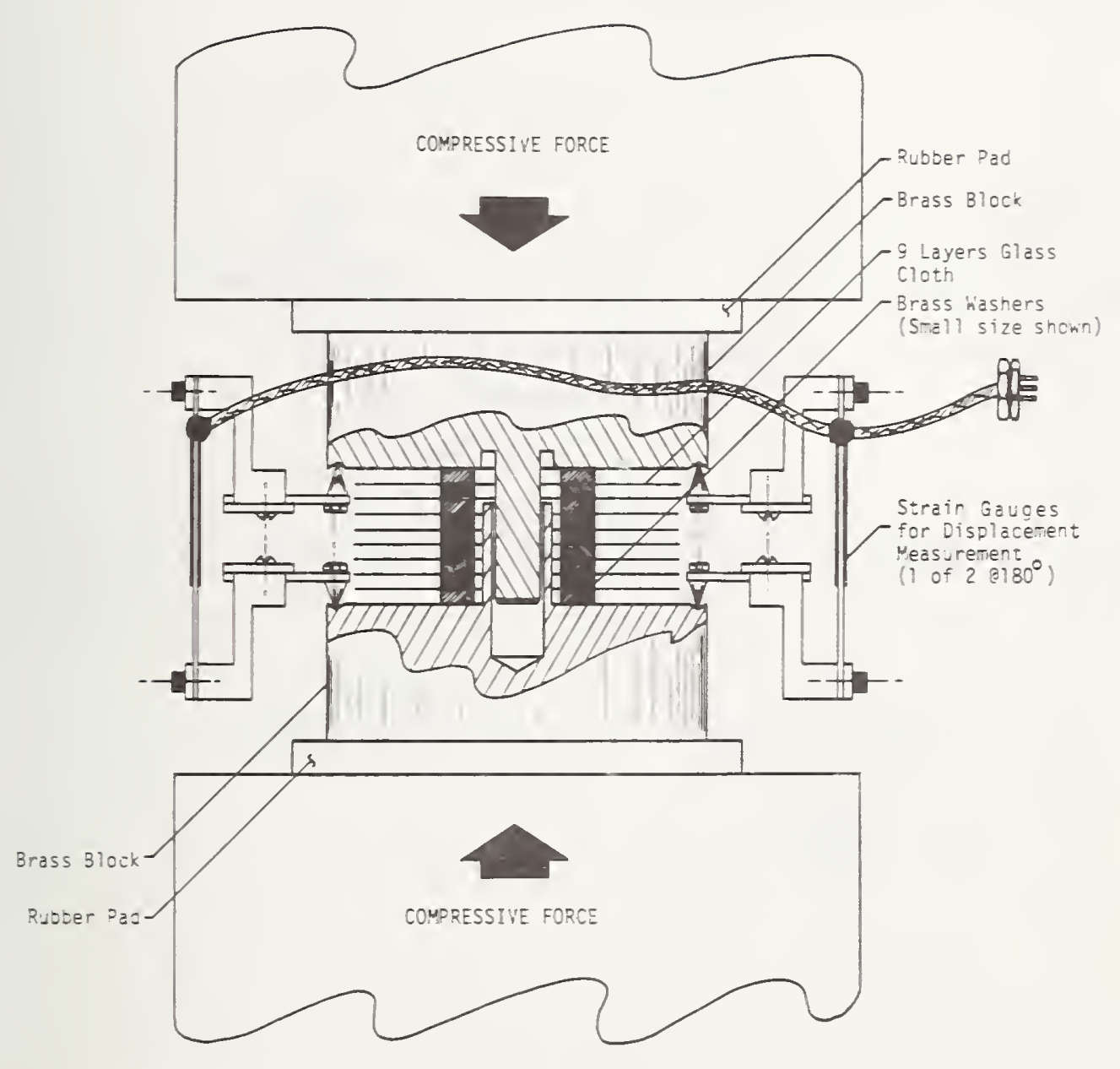

Figure 2-11. Glass cloth compressibility test apparatus. 


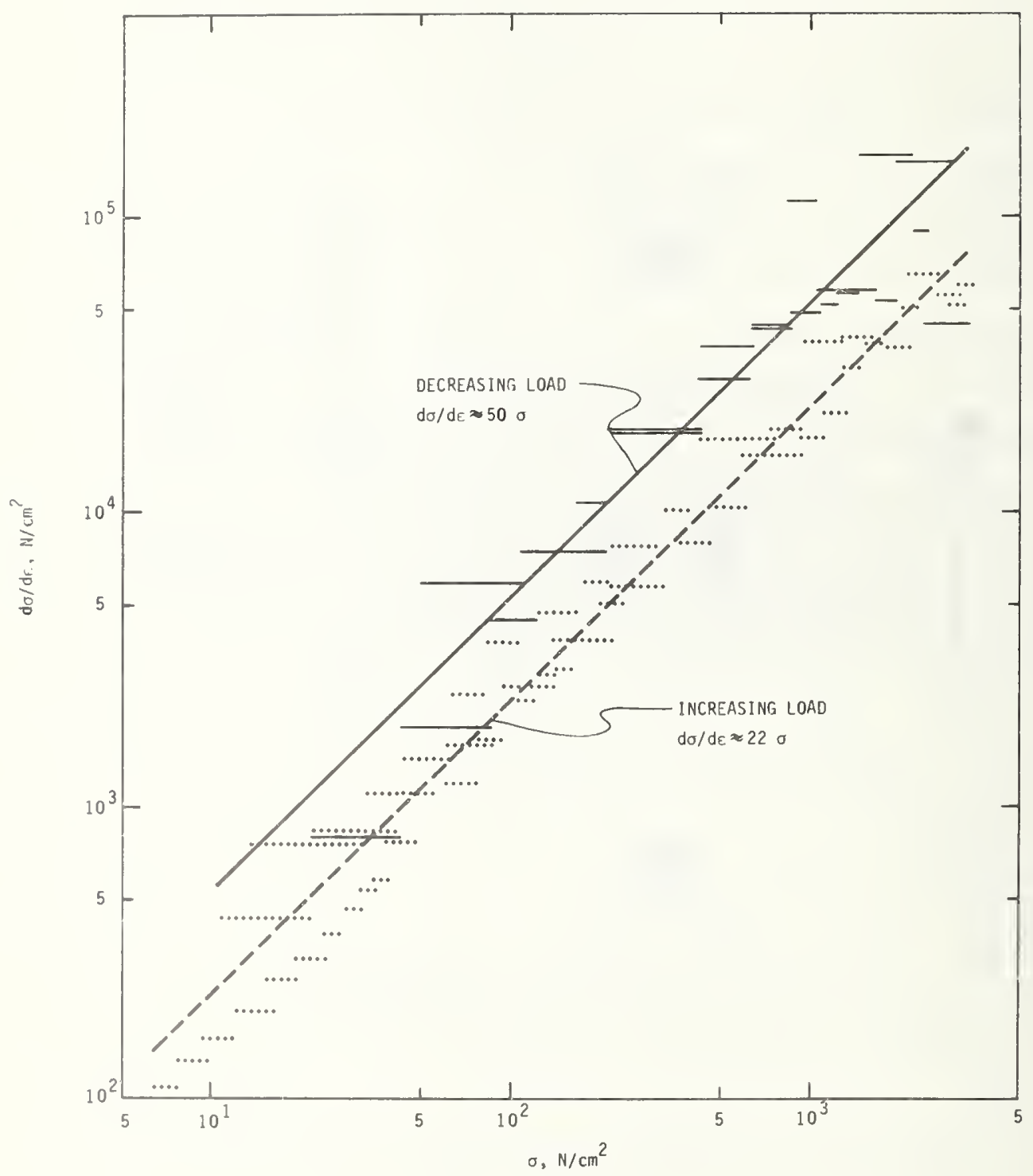

Figure 2-12. Compressibility of the fiberglass cloth as a function of applied stress. 


$$
\begin{array}{ll}
\frac{d \sigma}{d \varepsilon}=22 \sigma, & \text { for increasing loads } \\
\frac{d \sigma}{d \varepsilon}=50 \sigma, & \text { for decreasing loads. }
\end{array}
$$

These expressions cannot be valid as $\sigma \rightarrow 0$, since an infinite strain would be predicted. To compute the cumulative strain, assume that, for increasing stress

$$
\begin{array}{ll}
\frac{d \sigma}{d \varepsilon}=22 \sigma_{0}, & \sigma<\sigma_{0} \\
\frac{d \sigma}{d \varepsilon}=22 \sigma, & \sigma>\sigma_{0}
\end{array}
$$

where $\sigma_{0}$ is a very small stress below which elastic behavior does occur. Presumably it is near or below the lowest stress plotted in Figure 2-12. Then, for $\sigma>\sigma_{0}$, integration gives

$$
\varepsilon=\frac{1+\log \left(\sigma / \sigma_{0}\right)}{22} .
$$

The following table gives the total strain at an applied stress of $10 \mathrm{MN} / \mathrm{m}^{2}$, for several assumed values of $\sigma_{0}$.

$$
\sigma_{0}\left(10^{4} \mathrm{~N} / \mathrm{m}^{2}\right)
$$

1

3

10

20

$$
\varepsilon\left(\sigma=10 \mathrm{MN} / \mathrm{m}^{2}\right)
$$

0.310

0.255

0.223

Thus, it is reasonable to estimate that at an applied load of $10 \mathrm{NN} / \mathrm{m}^{2}$ (typical of that which may be experienced during magnet winding) the nominal $0.004^{\prime \prime}$ thick cloth would compress by $0.001^{\prime \prime}-0.0015^{\prime \prime}$.

Inspection of the washers after a test in which the compressive load reached $50 \mathrm{MN} / \mathrm{m}^{2}$ revealed a clear imprint of the cloth in the form of a square array of small dots. Evidently the localized stress on the brass where the fibers crossed each other was high enough to cause slight yielding of the brass, about 100-200 MN/m ${ }^{2}$. On the other hand, microscopic inspection of the glass cloth revealed no evident damage to it. 
These tests have yielded useful data for the stress analysis of the sponsor's model coil done in Section 5 of this report. More tests should be run, on different cloth materials, in order to find whether this non-linear behavior is typical of cloth materials in general.

\section{REFERENCES}

1. Fowlkes, C. W., Angerhofer, P. E., Newton, R. N., and Clark, A. F., "Characterization of a Superconducting Coil Composite," NBSIR 73-349, National Bureau of Standards, Boulder, Co (December 1973).

2. Griffel, W., Handbook of Formulas for Stress and Strain, Frederick Ungar Publishing Co., New York (1966), p. 248.

3. Mikese11, R. P. and McClintock, R. M., "A Method of Measuring Shear Modulus From $-424^{\circ}$ to $70^{\circ} \mathrm{F}, "$ Advances in Cryogenic Engineering, Vol. 7, K-11, p. 509-513, Plenum Press, NY (1961).

4. Cryogenic Materials Data Handbook, Air Force Materials Laboratory, ML-TDR-64-280, August 1964 .

5. Weston, W. F., Naimon, E. R., and Ledbetter, H. M., Unpublished Results, NBS, Cryogenics Division, Boulder, CO. 


\section{THERMAL PROPERTIES}

The thermal conductivity of the composite coil material and the thermal expansion of one of its constituents, the Cu-NbTi superconducting wire, are reported in this section. The thermal conductivity is highly anisotropic and significantly lower, particularly in the transverse direction, than predicted in the first report. ${ }^{\star}$ This anisotropy contributes significantly to coil stresses as will be seen in Section 5. More accurate predictions here are still somewhat high. The thermal expansion of the wire behaves more or less as expected. Several thermal properties have yet to be measured as indicated in Table 1-1. The most immediately useful, specific heat, is partially reported here.

\subsection{Thermal Conductivity of the Coil Composite}

Thermal conductivity measurements were performed on longitudinal and transverse sections of the superconducting coil composite. The superconducting coil is composed of a copper-stabilized niobium-titanium wire coil impregnated with epoxy. The cross-sectional area fractions perpendicular to the wires are: OFHC Cu/NbTi $=1.8$ and wire/epoxy $=3$. The longitudinal specimen was measured in direction 1, as illustrated in Figure 2-1 and the transverse specimen was measured in direction 2 (radial). No measurements were performed in the transverse-axial direction but estimates of the thermal conductivity in this direction are calculated. The thermal conuctivity specimens are shown in Figure 3-1.

The measurements were performed in a fixed-point apparatus designed to measure technically important materials to engineering accuracy. This apparatus requires less measurement time anà smaller specimens, but is inherently less accurate, than the variabletemperature apparatus used for nearly a decade to measure thermal and electrical properties of solids in the Cryogenics Division. The design accuracy of the new apparatus for midrange conductors is $\pm 10 \%$. Preliminary tests with standard reference materials indicate that the design accuracy has been achieved. This apparatus is used to measure the thermal conductivities of solids near the temperatures of fixed-point baths. Measurement time and cost is about one-fourth that of the variable-temperature apparatus.

The fixed-point apparatus, shown schematically in Figure 3-2, is based on linear heat-flow and is a modification of the Forbes cut-bar apparatus. The specimen heater and the differential-temperature thermocouples are permanently mounted on the copper end pieces, to which the specimen is attached. This eliminates the time-consuming task of * The references in each chapter are treated separately. 


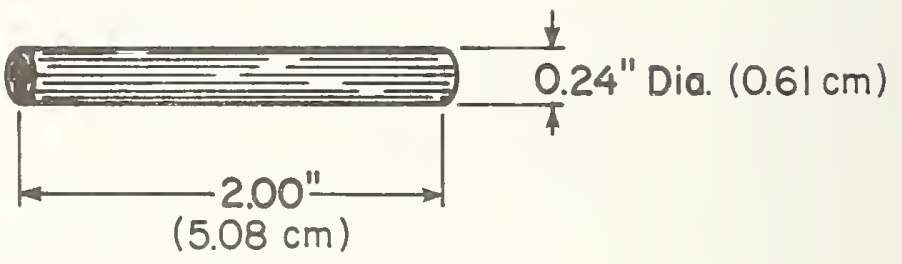

LONGITUDINAL

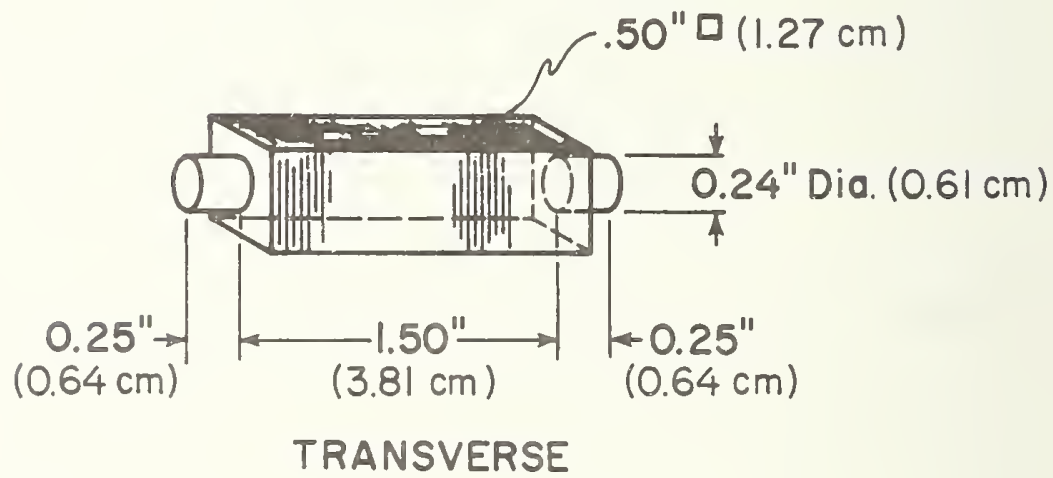

Figure 3-1. Coil composite thermal conductivity specimens. 


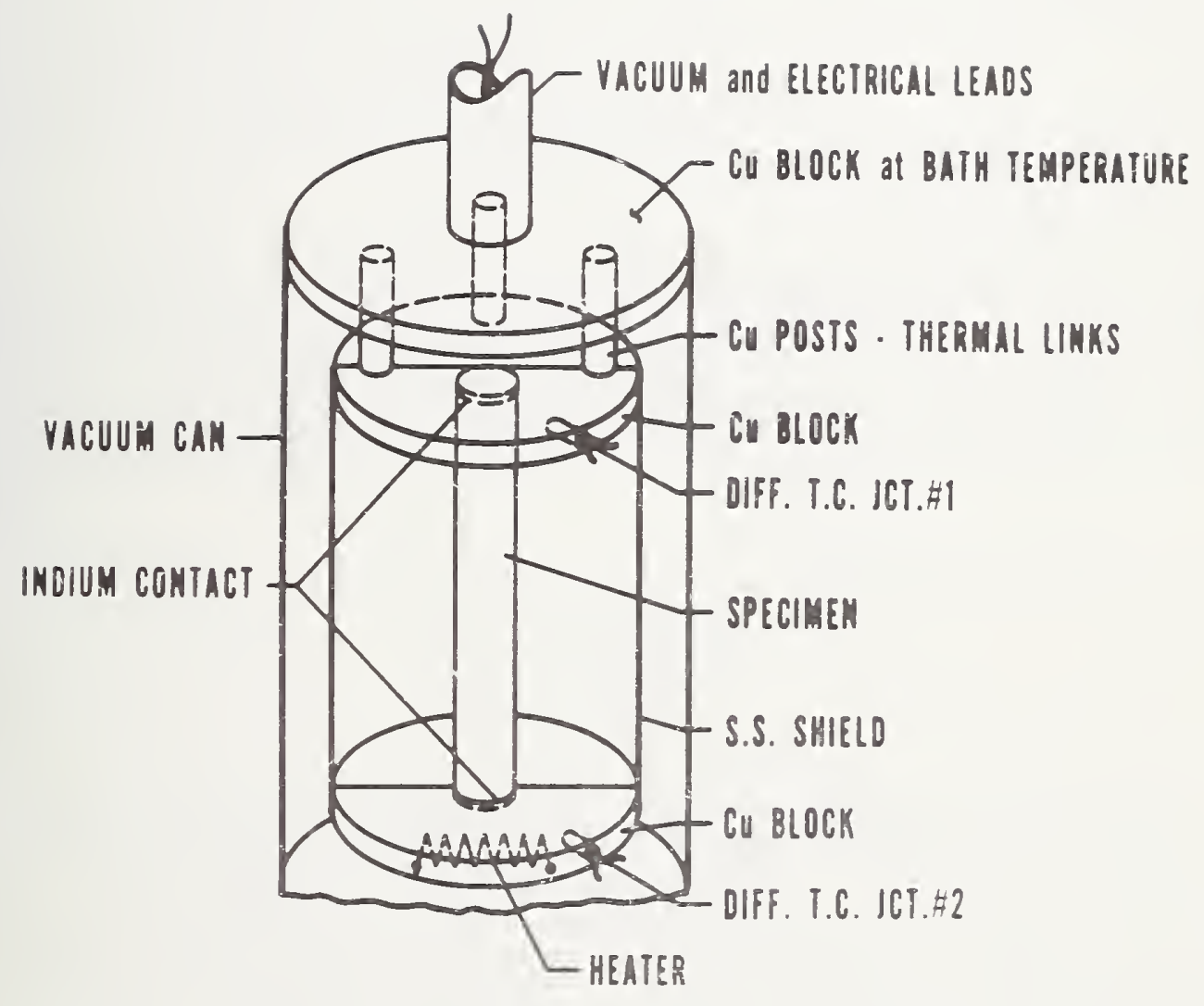

Figure 3-2. Thermal conductivity fixed temperature point apparatus. 
connecting these elements to each specimen, but also leads directly to the larger uncertainty of this apparatus. Specimen assembly involves only the relatively simple task of soldering (woods alloy or indium is used) the specimen and vacuum can in place. The differential-temperature observed includes not only the specimen temperature gradient but also the temperature difference caused by heat flow through the specimen end sections, the soldered thermal contact, and the copper end pieces. These latter temperature differences are generally less than $15 \%$ of the total and can be estimated sufficient1y accurately to reduce total uncertainty to below $\pm 10 \%$.

The principle of operation is as follows. For each fixed-point bath, a series of empty chamber gradients are established to determine $\dot{Q}_{0}=f(\Delta T)$ of the specimen chamber. This chamber, shown as a cylinder in Figure 3-2, is actually a bellows to reduce both strain on the specimen and the values of $\dot{Q}_{0}$. Plots of $\dot{Q}_{0}$ versus $\Delta T$ are obtained for each of the baths used. $\dot{Q}_{0}$ is the electrical power input required to establish the temperature differential, $\Delta \mathrm{T}$. Since the empty chamber configuration remains permanently fixed, the above procedure is performed only once.

A specimen is then installed and the power input to establish a temperature-differential, $\Delta \mathrm{T}$, is determined. This $\Delta \mathrm{T}$ is generally small compared to $\mathrm{T}$, but if the specimen thermal conductivity varies weakly or linearly with temperature, this restriction can be relaxed. The heat-flow through the specimen, $\dot{Q}-\dot{Q}_{0}=\dot{Q}-f(\Delta T)$, is determined and thermal conductivity of the specimen is calculated from

$$
\lambda=\frac{[\dot{\mathrm{Q}}-\mathrm{f}(\Delta \mathrm{T})] \ell}{\mathrm{A} \Delta \mathrm{T}},
$$

where $l$ is the length of the specimen between the copper end pieces and A is the crosssectional area of the specimen. Corrections are applied to the measured $\Delta \mathrm{T}$ to account for the temperature gradients in the specimen ends and clamps. These estimates are based in part on qualitative arguments of the heat-flow streamlines at the ends of the specimen. Detailed heat flow calculations based on the solution of the general heat flow equation are not performed, because the magnitude of the correction does not warrant such complexities. In addition, these corrections are based on check measurements of NBS standard reference materials of thermal conductivity. The correction for a conventional cylindrical, isotropic, heterogeneous specimen is estimated as $\pm 10 \%$. 
Principal sources of error are the end effects discussed above, thermocouple callbration errors, bath temperature drift, and heat loss by radiation and gaseous conduction. For specimens with diameters which are small compared to the specimen chamber diameter $(3.2 \mathrm{~cm})$, the effect of the radiation heat transfer is nearly equal in the $\dot{Q}_{\text {and }} \dot{Q}_{0}$ determinations and, therefore, is diminished in the calculation of $\lambda$. Temperature drift and radiation errors will be greatest for large diameter specimens. The optimum range of conductivities measureable with this apparatus is about the same as with the variabletemperature apparatus, 0.001 to $50 \mathrm{~W} / \mathrm{cm} \mathrm{K}$. Conductivities outside of this range can be measured with reduced accuracy. Calibration of this apparatus has been performed by measuring the thermal conductivity of NBS Standard Reference Materials, SRM's, 734 and 735. These SRM's were certified for thermal conductivity, to approximately $2 \%$ accuracy, using the variable-temperature apparatus.

The results of the measurements on the longltudinal coll composite specimen are given in Tatle 3-1 and Figure 3-3. Corrections have been applied to the measured data to compensate for end effects in the apparatus. These corrections are based on measurements on standard reference materials and decrease the measured values by $7 \%$ for this specimen. The uncertainty in the corrected data is $\pm 15 \%$. The interpolated curve given for the longitudinal specimen in Figure 3-3 is based on the typical temperature dependence of OFHC copper.

It is interesting to compare the measured data to values'calculated from the properties of the constituents of the coil. The longitudinal specimen can be separated into three parallel components, $\mathrm{Cu}, \mathrm{NbTi}$, and Epoxy. The corresponding relative crosssectional areas of these are $0.47,0.26$, and 0.27 , respectively. The effective conductivity of parallel specimens is obtained by summing the area-weighted conductivities of each of the parts. The estimated values of the conductivities of $\mathrm{Cu}$, NbTi, and epoxy, listed in Table 3-1, were obtained from Childs, et al. ${ }^{2}$ The uncertainties of these values are high because of the lack of adequate characterization data. OFHC copper is the wire matrix material, but the fabrication procedure may have considerably altered its composition. The low-temperature conductivity of pure metals is significantly dependent on this composition and may also be altered. The calculated values given in Table 3-1 differ from the observed values by as much as $23 \%$. These differences are considered reasonable in view of the large uncertainties in defining the actual nature of the constituents. 
Table 3-1 Thermal Conductivity of Longitudinal and T ransverse Sections of Superconducting Coil Composite

\begin{tabular}{|c|c|c|c|c|c|c|}
\hline Specimen & $\begin{array}{l}\text { Temp. } \\
(\mathrm{K})\end{array}$ & $\begin{array}{c}\lambda_{\text {obs }} \\
(\mathrm{W} / \mathrm{mK})\end{array}$ & $\begin{array}{c}\lambda_{\text {calc }} \\
(\mathrm{W} / \mathrm{mK})\end{array}$ & $\begin{array}{c}\lambda_{\mathrm{cu}} \\
(\mathrm{W} / \mathrm{mK})\end{array}$ & $\begin{array}{c}\lambda \\
\text { epoxy } \\
(\mathrm{W} / \mathrm{mK})\end{array}$ & $\begin{array}{l}\lambda_{\mathrm{NbTi}} \\
(\mathrm{W} / \mathrm{mK})\end{array}$ \\
\hline \multirow[t]{4}{*}{ Longitudinal } & 4.90 & 169 & 136 & 290 & 0.05 & 0.1 \\
\hline & 78.8 & 201 & 257 & 550 & 0.92 & 4 \\
\hline & 196 & 165 & 187 & 400 & 1.62 & 6 \\
\hline & 276 & 169 & 187 & 400 & 1.82 & 10 \\
\hline \multirow{4}{*}{$\begin{array}{l}\text { Transverse } \\
\text { (radial) }\end{array}$} & 7.50 & 0.24 & 0.20 & $10 *$ & 0.05 & - \\
\hline & 78.7 & 0.81 & 0.92 & $10 *$ & 0.09 & - \\
\hline & 197 & 1.13 & 1.33 & $10 *$ & 0.16 & - \\
\hline & 276 & 1.28 & 1.33 & $10 *$ & 0.18 & - \\
\hline \multirow{4}{*}{$\begin{array}{l}\text { Transverse } \\
\text { (axial) }\end{array}$} & 7.50 & - & 0.91 & - & - & - \\
\hline & 79.0 & - & 3.3 & - & - & - \\
\hline & 197 & - & 4.3 & - & - & - \\
\hline & 277 & - & 4.3 & - & - & - \\
\hline
\end{tabular}

These values are for the transverse direction of Copper - NbTi composite (see text)。 


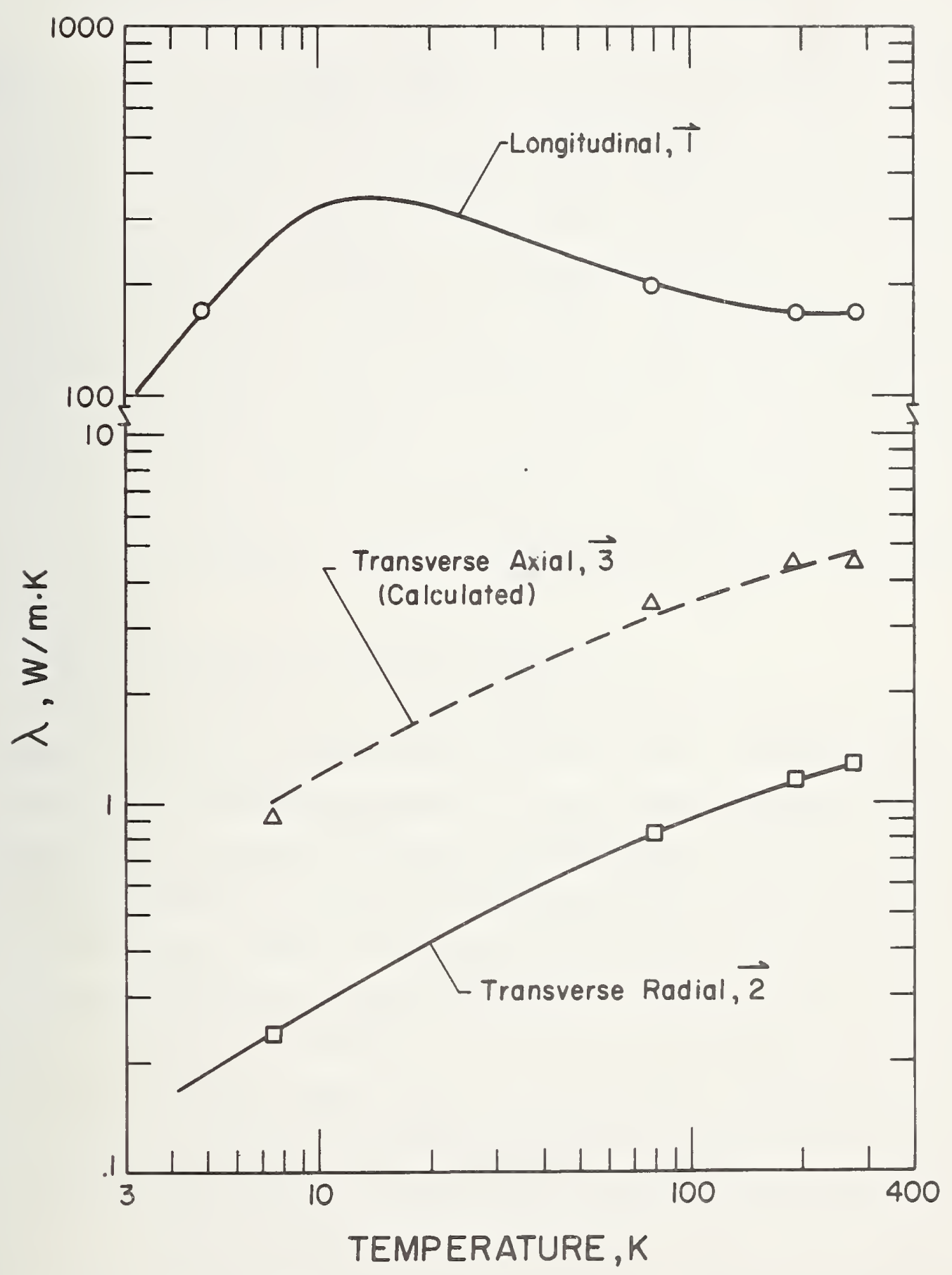

Figure 3-3. Thermal conductivity of coil composite materials. (Interpolated curves are based on the temperature dependence of similar materials.) 
The observed thermal conductivities for the transverse (radial) specimen are also given in Table 3-1 and Figure 3-3. These values required no end effect corrections and the uncertainty is $\pm 10 \%$. Again the solid curve is based on the temperature dependence of similar materials.

The transverse specimens can be viewed as two parallel segments. The first is composed of the epoxy strip directed along the specimen axis. The second is composed of epoxy and wire segments arranged in series with the heat flow direction. The dimensions of the two parallel conductivity paths are different for the radial and axial transverse sections. The epoxy strip for the radial transverse specimen has a relative cross-sectional area of 0.04. The epoxy-wire segmented strip is 0.96 of the total area. As before the conductivity of the two parallel paths is obtained by summing the component conductivities weighted by their respective relative areas. However, first one must obtain the effective conductivity of the epoxy-wire segmented portion. The effective conductivity of segments arranged in series can be obtained by summing the thermal resistances of the various segments. Since the cross-sectional areas of all the segments are equal, it can be shown that

$$
\frac{1}{\lambda}=\frac{l_{1}}{\lambda_{1}}+\frac{l_{2}}{\lambda_{2}}+\ldots
$$

where $l_{i}$ is the relative length of the $i^{\text {th }}$ segment in the direction of heat flow. According to reference 1 the relative length of epoxy in this radial direction is 0.2 and that of the wire is 0.8 . It would be desireable to further divide the wire into the $\mathrm{Cu}$ and NbTi components, but because of insufficient characterization data, this is not done in the transverse case. Instead we just make a crude estimate of the effective conductivity of the wire as $10 \mathrm{~W} / \mathrm{mK}$ at all temperatures. The calculated values for the transverse (radial) specimen are tabulated in Table $3-1$.

Because of the reasonable agreement obtained it may be useful to estimate values for the transverse-axial case by the same technique. The results are $0.91,3.3,4.3$, and $4.3 \mathrm{~W} / \mathrm{mK}$ at $7.5,79,197$, and $277 \mathrm{~K}$, respectively. These values are also shown in Figure $3-3$.

The thermal conductivity is the most highly anisotropic of all the properties measured on the coil composite. The longitudinal value being a factor of about 1000 times greater than the poorest conducting transverse value in the radial direction. Even the axial and 
radial thermal conductivities differ by a factor of 10. These differences are not unexpected because of the high thermal conductivity of copper compared to epoxy and the obvious anisotropy in conducting paths.

These differences will be of no consequence in steady state operation but will have a significant effect on any transient behavior. The thermal contraction (and its anisotropies as well) creates significant thermal stresses during cooldown and these can be compounded by the anisotropy of heat removal. The final residual stress distribution due to contraction from cooling to $4 \mathrm{~K}$ will not be affected but significant differential stresses may occur in the coil depending upon the rate of heat removal. Also, propagation of a normal conducting zone in a wire will tend to be along the wire instead of the desired transverse path to the cooling surface. The buildup of local stresses due to this local heating and anisotropic heat removal is difficult to assess but may be significant, particularly if repetitive. These effects could be mitigated with an appropriate filler in the epoxy to increase the conductivities transverse to the wires and reduce the anisotropy. Measurements of thermal diffusivity could quantify the magnitude of these effects and permit an assessment of their significance.

\subsection{Thermal Expansion of the Superconducting Wire}

The superconducting wire properties are essential components of the composite analysis and thermal contraction of the wire during cooldown is one of the major sources of stress. Toward this end the low-temperature thermal expansion of the Cu-NbTi wire was measured using a quartz tube dilatometer from room temperature to $4 \mathrm{~K}$ utilizing the apparatus of Clark. ${ }^{3}$ The dilatometer is schematically shown in Fig. 3-4. The experimental procedure for the wires was the same as the procedure used by McConnell, et al for measuring fibers. The specimen tested was the superconducting wire which is one of the constituent materials of the NSRDC coil composite. The wire specimen was attached to a flanged copper plate and had 3 individual wires soldered on a $1 \mathrm{~cm}$ diameter, spaced at $120^{\circ}$ intervals. The lower attachment for the wires is a solid brass disc, upon which the quartz rider sits. The specimen length was $179.0 \pm 2.5 \pm(7.04 \pm .10$ inches). The specimen is shown in Figure 3-5. The wires themselves are 180 filament, $.056 \times .072 \mathrm{~cm}(.022 \times .028$ in $)$ drawn and annealed wire with $\mathrm{Cu} / \mathrm{S} . \mathrm{C}$. ratio of $1.8 / 1.0$ and a twist of about $1 / \mathrm{cm}(2.4 / \mathrm{in})$.

Since this type of thermal expansion measurement is relative, it must be compared to a specimen of known material. The calibration was accomplished by measuring several times 


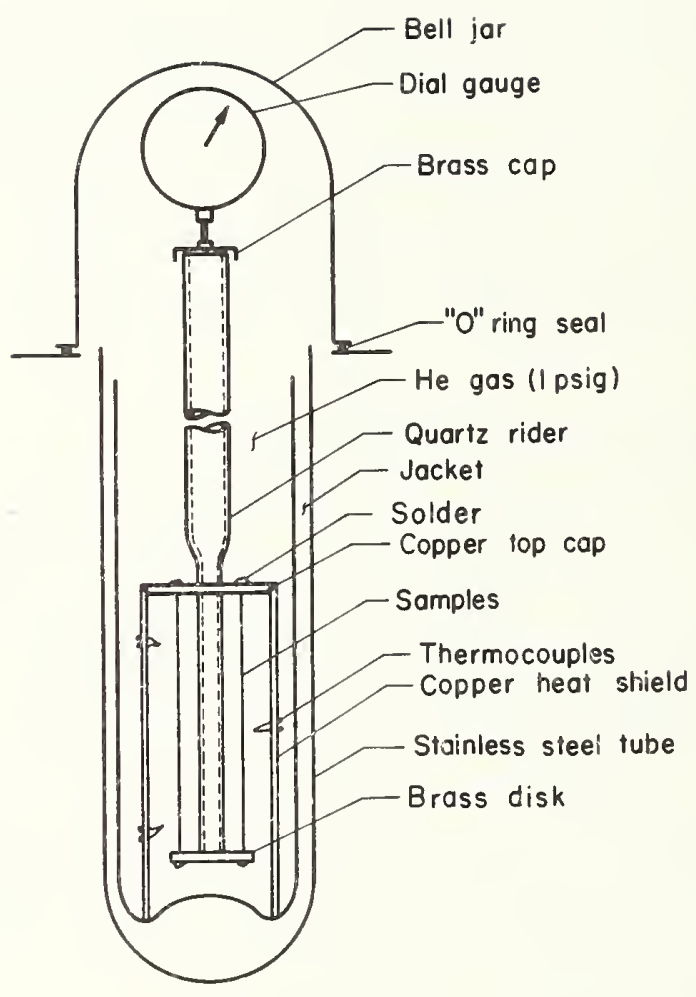

Figure 3-4. Dilatometer schematic. 


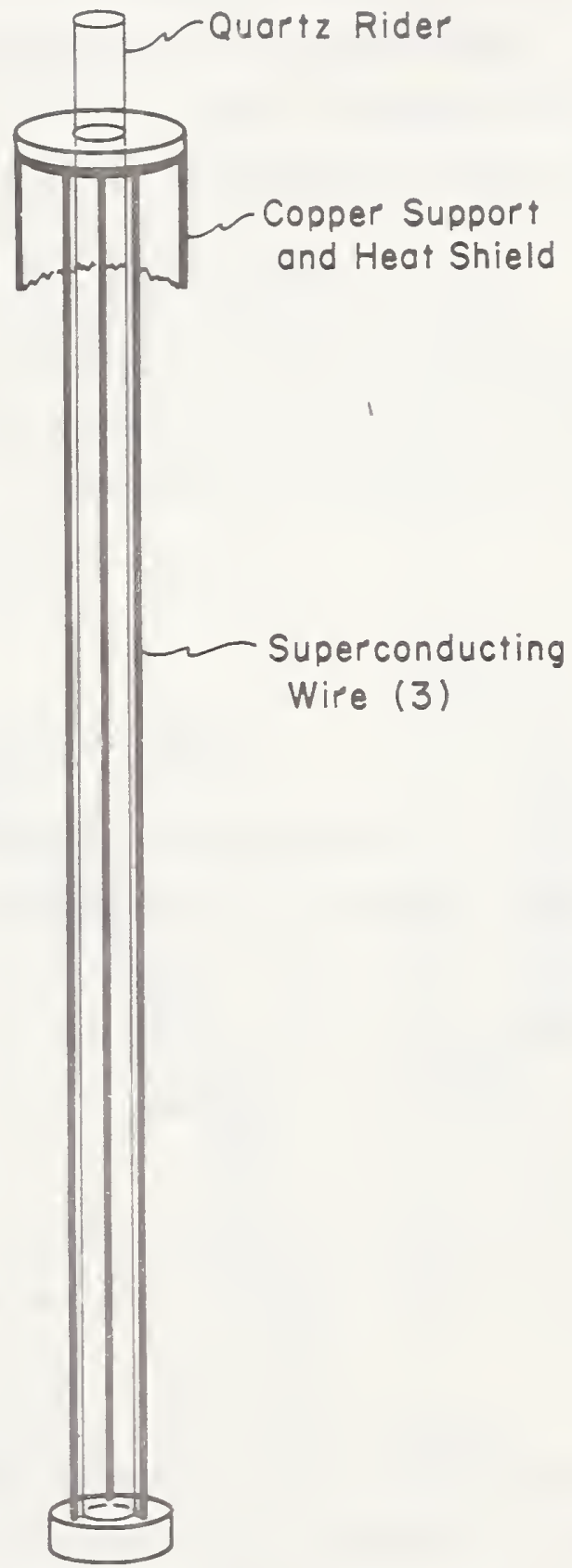

Figure 3-5. Superconducting wire thermal expansion specimen. 
a specimen machined from OFHC copper. The difference between the average of the calibration data and the standard reference data ${ }^{5}$ was due to the differential expansion between the quartz tubes and the copper heat shield. This difference was applied to the specimen measurement as a correction factor. The correction was 1 ess than $10 \%$ of the copper values throughout the entire temperature range.

The observed strain in the sample is

$$
\Delta \mathrm{L}_{\mathrm{OBS}} / \mathrm{L}_{\mathrm{o}}=\left(\Delta \mathrm{L}_{\mathrm{Cu}}-\Delta \mathrm{L}_{\mathrm{s}}+\Delta \mathrm{L}_{\mathrm{c}}\right) / \mathrm{L}_{\mathrm{o}}
$$

where $\Delta \mathrm{L}_{\mathrm{S}} / \mathrm{L}_{\mathrm{O}}$ is the sample contraction, $\Delta \mathrm{L}_{\mathrm{Cu}} / \mathrm{L}_{\mathrm{O}}$ the reference data contraction of the OFHC copper heat shield, and $\Delta \mathrm{L}_{\mathrm{c}} / \mathrm{L}_{\mathrm{O}}$ is the correction factor determined by measuring the sample of OFHC copper. For this calibration measurement of copper

$$
\Delta \mathrm{L}_{\mathrm{S}} / \mathrm{L}_{\mathrm{o}}=\Delta \mathrm{L}_{\mathrm{Cu}} / \mathrm{L}_{\mathrm{O}}
$$

and

$$
\Delta \mathrm{L}_{\mathrm{OBS}} / \mathrm{L}_{\mathrm{o}}=\Delta \mathrm{L}_{\mathrm{c}} / \mathrm{L}_{\mathrm{O}}
$$

This correction arises from the difference between the contraction of the quartz support tube and the copper heat shield over the $2 \mathrm{~cm}$ segment between the end of the quartz rider and the bottom of the support tube. From the known expansion of these materials, the predicted correction curve and the measured correction curve can be compared as shown in Fig. 3-6. The measured curve is an average of four calibration measurements of a high purity copper sample.

This correction applied to the two measurements of the wire sample results in the averaged data shown in Fig. 3-7 obtained from equation (3-3) rewritten as

$$
\Delta \mathrm{L}_{\mathrm{S}} / \mathrm{L}_{\mathrm{O}}=\left(\Delta \mathrm{L}_{\mathrm{Cu}}-\Delta \mathrm{L}_{\mathrm{OBS}}+\Delta \mathrm{L}_{\mathrm{c}}\right) / \mathrm{L}_{\mathrm{o}} \cdot
$$

This averaged data was fit with a polynomial limited to $\mathrm{T}^{5}$ and the values of the expansion and its derivative, the expansion coefficient, are shown in Table 3-2. at standard temperature intervals. Because of the accumulation of errors arising from the summation in equation (3-6), the estimated uncertainty from experimental deviations is about $\pm 10 \times 10^{-5}$ from the expansion and $\pm 1 \times 10^{-6} \mathrm{~K}^{-1}$ or the expansion coefficient. Variations due to inhomogeneities in the wire would not be expected to be this large. 


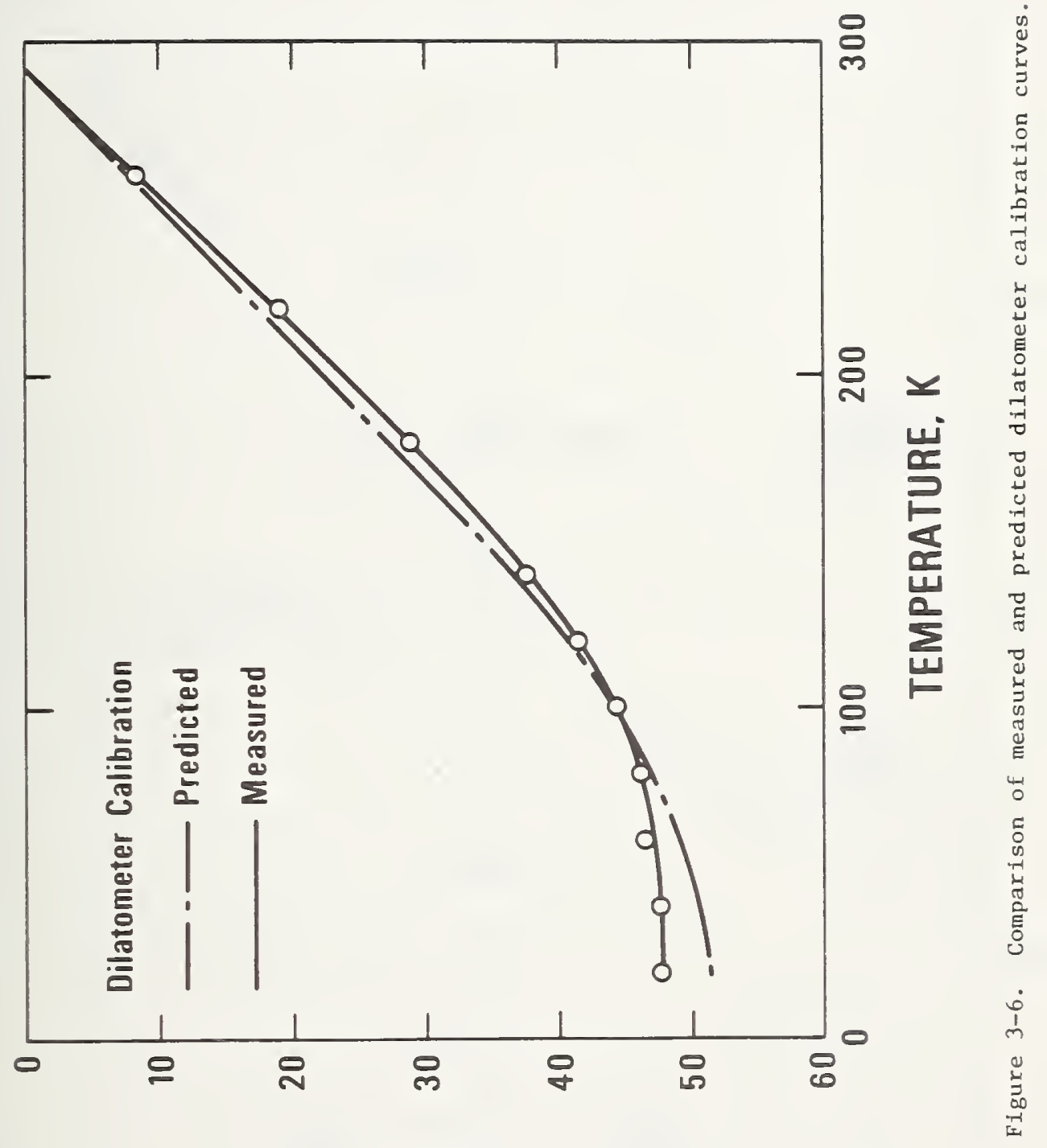

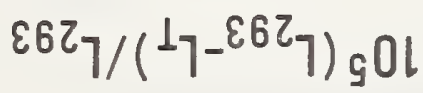




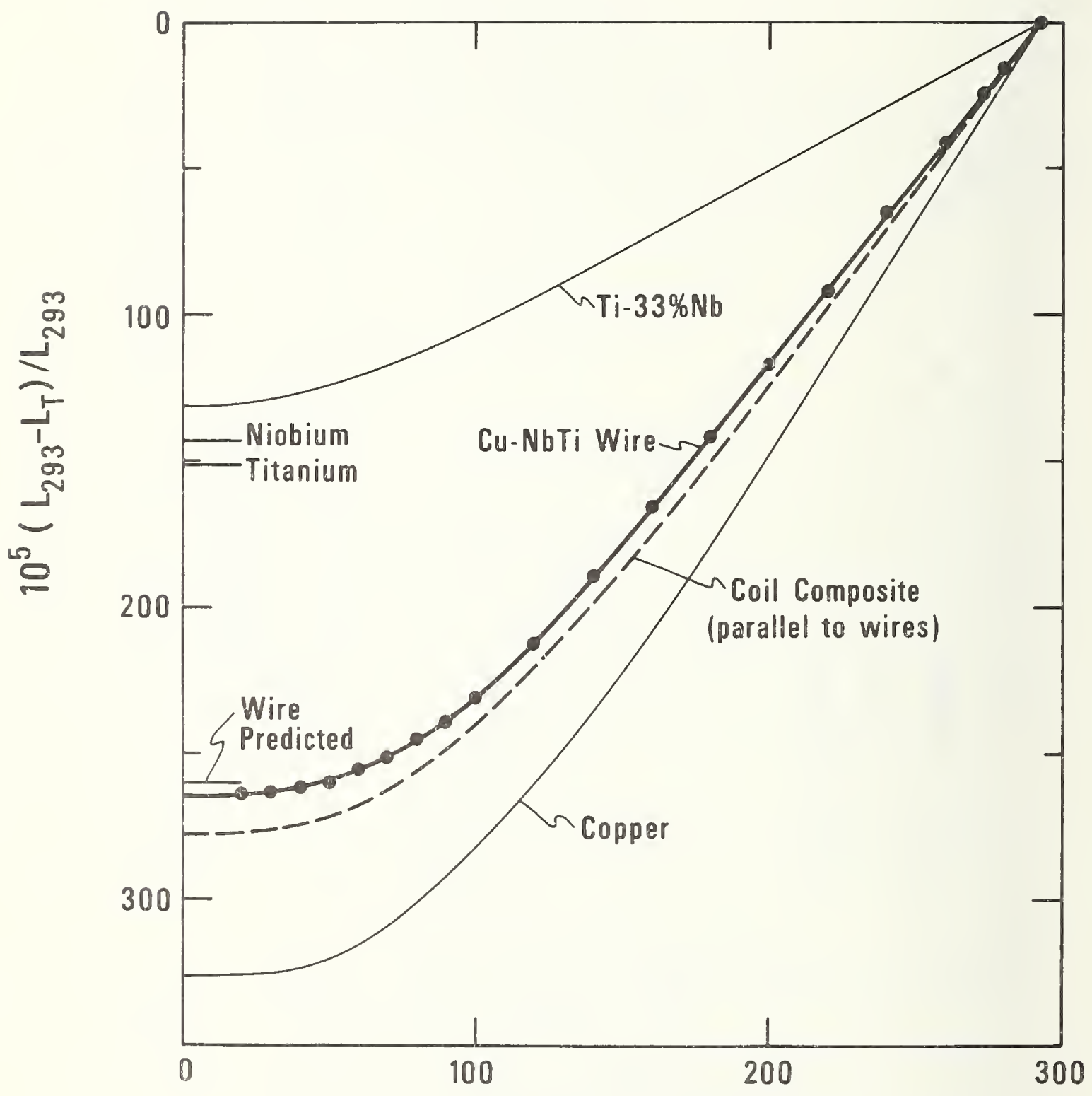

\section{TEMPERATURE, $\mathrm{K}$}

Figure 3-7. The thermal contraction of Cu-NbTi wire compared to niobium, titanium, copper, and the longitudinal coil composite. 
Table 3-2. Thermal Expansion of Copper-Niobium Titanium Supercondicting Wire

\begin{tabular}{|c|c|c|}
\hline $\begin{array}{c}\text { Temperature } \\
(\mathrm{K})\end{array}$ & $10^{5} \frac{\mathrm{L}_{293^{-\mathrm{L}_{\mathrm{T}}}}}{\mathrm{L}_{293}}$ & $\frac{10^{6}}{\mathrm{~L}_{293}} \frac{\mathrm{dL}}{\mathrm{dT}}, \mathrm{K}^{-1}$ \\
\hline 0 & $265 a$ & $0.0 \mathrm{a}$ \\
\hline 10 & $265 a$ & $0.16 a$ \\
\hline 20 & 265 & $0.60 a$ \\
\hline 30 & 264 & 1.3 \\
\hline 40 & 262 & 2.1 \\
\hline 50 & 259 & 3.1 \\
\hline 60 & 256 & 4.2 \\
\hline 70 & 251 & 5.2 \\
\hline 80 & 245 & 6.3 \\
\hline 90 & 238 & 7.4 \\
\hline 100 & 231 & 8.4 \\
\hline 120 & 212 & 10.1 \\
\hline 140 & 190 & 11.2 \\
\hline 160 & 167 & 11.8 \\
\hline 180 & 142 & 12.2 \\
\hline 200 & 117 & 12.4 \\
\hline 220 & 91 & 12.5 \\
\hline 240 & 66 & 12.6 \\
\hline 260 & 4.1 & 12.7 \\
\hline 273 & 25 & 12.8 \\
\hline 280 & 16 & 12.8 \\
\hline 293 & 0 & $12.9 a$ \\
\hline 300 & $-9 a$ & $12.9 a$ \\
\hline
\end{tabular}


We can now compare the results with previous measurements of the coil composite ${ }^{1}$ and with measurements of the constituents of the wire. There is no data ava1lable on the thermal expansion of the $\mathrm{Nb}-48 \% \mathrm{Ti}$ which is used in the superconducting wire. Data are avallable, however, for niobium ${ }^{6}$, titanium ${ }^{6}$, $\mathrm{Ti}-33 \% \mathrm{Nb}^{7}$, and copper ${ }^{5}$. The thermal expansion curve of the coil composite in the direction parallel to the wires is shown also in Fig. 3-7 and is just slightly larger than that of the wires. This would be expected since the coll expansion should be dominated by the wires but slightly enlarged by the weaker epoxy whose thermal expansion coefficient is significantly larger.

The thermal expansion curve of $\mathrm{T} 1-33 \% \mathrm{Nb}$ is also shown 1 . F1g. $3-7$ as well as the total length change to $0 \mathrm{~K}$ for $\mathrm{Ti}$ and $\mathrm{Nb}$. As a first approximation to the composite wire value whose copper-to-superconductor ratio $1 \mathrm{~s} 1.8: 1$, the total length change to $0 \mathrm{~K}$ would be (1.8 $\left.\Delta \mathrm{L}_{\mathrm{Cu}}+1 \Delta \mathrm{L}_{\mathrm{Ti}-\mathrm{Nb}}\right) / 2.8$ where the $\Delta \mathrm{L}_{\mathrm{Ti}-\mathrm{Nb}}$ is an average of the data avallable. This predicted value of the total length change to $0 \mathrm{~K}$ is also shown $\mathrm{In} \mathrm{F}$. the curve would be similar to the others) and the $2 \%$ difference from the measured value 1 s well within the experimental error. Since the thermal expansion of alloys appears to be well behaved at low temperatures unless phase transformations are involved ${ }^{3}$ this $^{1 s}$ probably a safe procedure to predict the thermal expansion for other wires from the copperto-superconductor ratio with an accuracy of the order of $10 \%$.

\subsection{Specific Heat of the Coll Composite and Fiberglass Epoxy}

The specific heat at low temperatures was measured for both the superconducting coil composite and the flberglass-epoxy composite. Both specimens were portions of the same material used for the thermal expanston measurements. ${ }^{1}$ The coil composite 1 s $27 \%$ fiberglass epoxy by volume and the fiberglass-epoxy specimen was constituted to have the same proportlons of flberglass and epoxy as in the coll composite. The measurements were taken only to about $25 \mathrm{~K}$ and either further measurements or extrapolations w111 be done to extend the data to room temperature. The measurements reported here were performed by E. W. Collings of Battelle Columbus Laboratories, Columbus, $\mathrm{OH}$.

The specific heat at low temperature measured here was by adiabatic calorimetry (the Nernst method). In this method, small increments of heat are supplied to a thermally insulated sample, and the accompanying temperature increase is measured. The capac1ty 1s calculated using the relationship: 


$$
C_{p}=\frac{d Q}{d T} \simeq \frac{\Delta Q}{\Delta T}=\frac{I^{2} R \Delta t}{\Delta T}
$$

where current, I, is supplied for a time-interval, $\Delta t$, to a heater winding of resistance, R, in thermal contact with the sample; and $\Delta T$ is its resultant rise in temperature. The experiment is of course carried out at constant pressure, hence the subscript p. (However, in the temperature range of interest the difference between $C_{p}$ and $C_{v}$ is negligible).

In general, a calorimeter can is immersed in liquid helium during the course of the experiment, and thermal insulation of the sample is achleved with the aid of a vacuum. Inside the calorimeter can the sample is secured to a copper block which carries a manganin heater (1900 ohms) and a calibrated germanium resistance thermometer. In performing a measurement, a pulse of current is passed through the heater coil. The duration of the heat pulse (typically 6 to 60 seconds) Is measured by a clock also actuated by the current. A correction for heat leakage is applied to the data.

In a test of performance, a specimen of high-purity copper was measured yielding a fit of $\gamma=7.07 \mathrm{~mJ} / \mathrm{mole}-\mathrm{deg}^{2}$ and $\theta_{D}=338 \mathrm{~K}$ to the data. These values agree reasonably well with those reported in the literature. The accuracy of the heat-capacity results are estimated to be about $\pm 15 \%$.

The results of these measurements are shown in Flg. 3-8 and Tables $3-3,4$ and 5 . The tabular data is included to ald in future fitting and extrapolation. One can readily see the similar behavior except for the small discontinuity in the coil composite due to the superconducting-to-normal transition of the superconductor. This transition has been plotted on an expanded scale in Fig. 3-9 which reveals the broad nature of the critical temperature $\left(\mathrm{T}_{c}\right)$ transition centered about $9.2 \mathrm{~K}$. That the transition is several tenths of a degree wide implies that there are reglons of Cifferent $T_{c}$ due to cold working or diffusion of the superconductor itself.

The fiberglass-epoxy data is smooth and can be fit to a polynomial. The four term polynomial

$$
c=\alpha_{1}\left(\frac{T}{10}\right)+\alpha_{2}\left(\frac{T}{10}\right)^{2}+\alpha_{3}\left(\frac{T}{10}\right)^{3}+\alpha_{4}\left(\frac{T}{10}\right)^{4}
$$




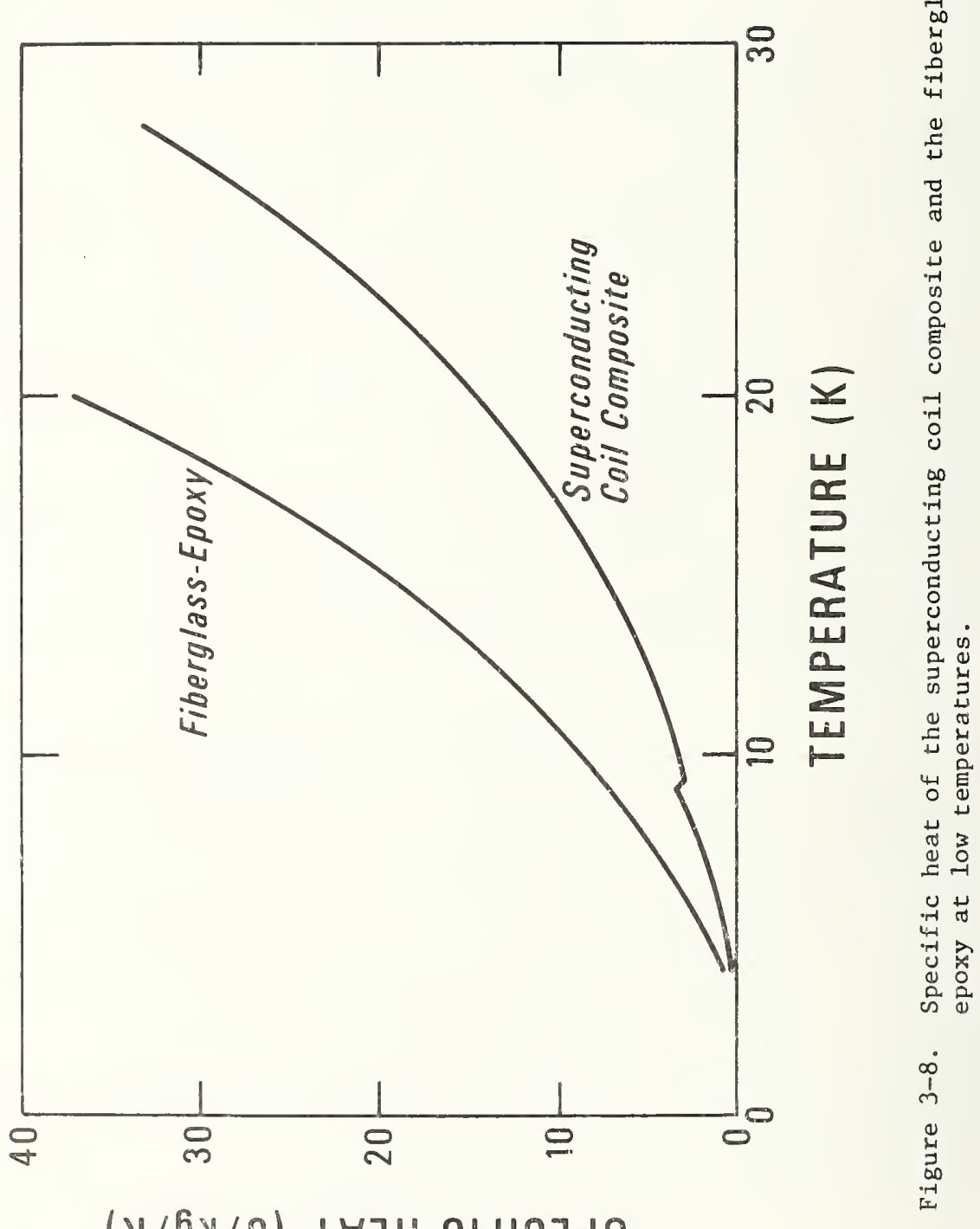

$(y / 6 y / 4) \perp \forall \exists H$ JIHIJJdS 


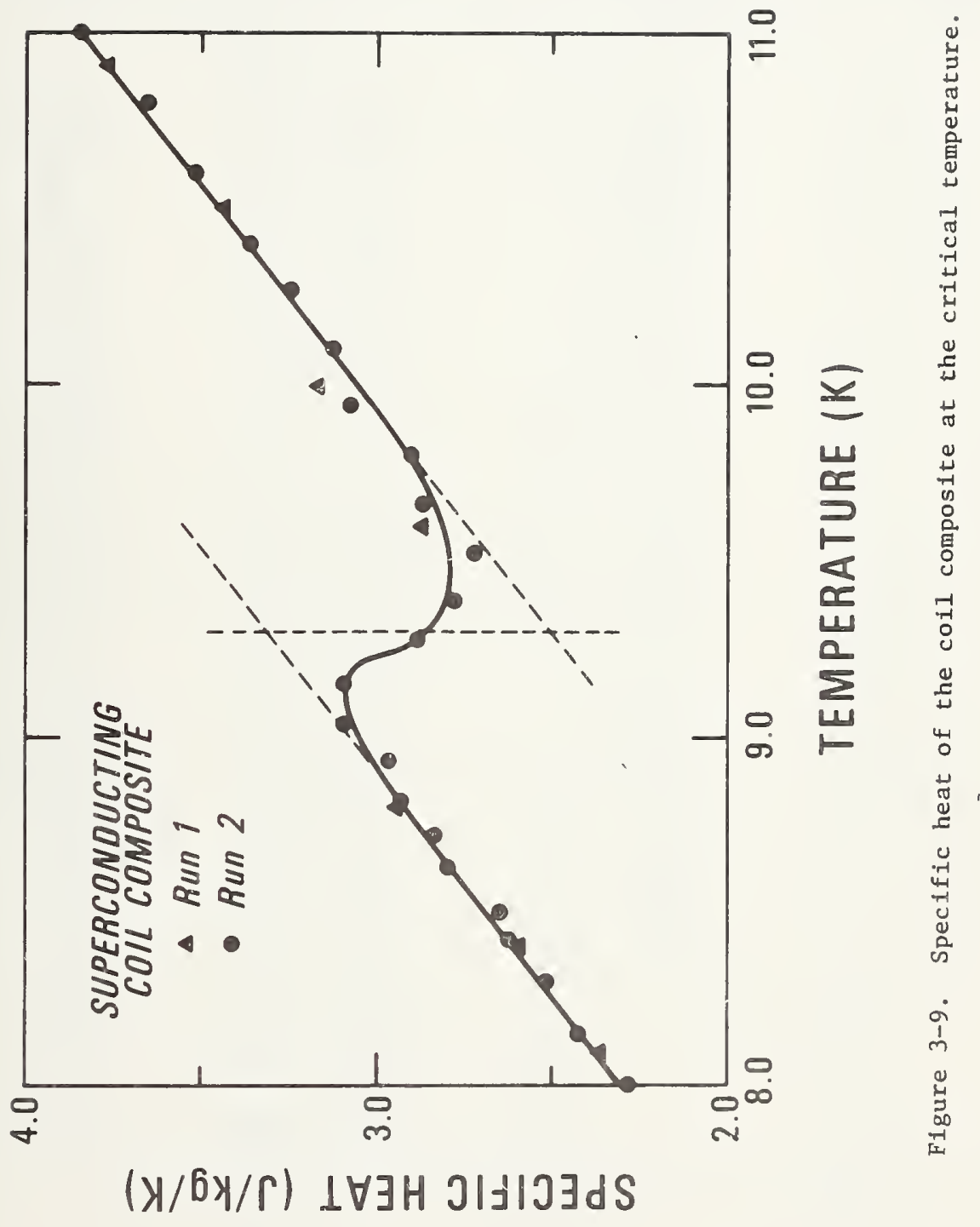


Table 3-3. Specific Heat of NbTi/Cu-Fiberglass Epoxy Coil Composite in Temperature Range 4.0 to 27.2 (Run No. 1)

\begin{tabular}{|c|c|c|c|}
\hline $\begin{array}{l}\text { Temperature } \\
(\mathrm{K})\end{array}$ & $\begin{array}{c}\text { Specific Heat } \\
(\mathrm{J} / \mathrm{kg}-\mathrm{K})\end{array}$ & $\begin{array}{l}\text { Temperature } \\
(K)\end{array}$ & $\begin{array}{l}\text { Specific Heat } \\
(\mathrm{J} / \mathrm{kg}-\mathrm{K})\end{array}$ \\
\hline 4.0 & 0.338 & 9.2 & 3.060 \\
\hline 4.2 & 0.381 & 9.6 & 2.868 \\
\hline 4.5 & 0.464 & 10.0 & 3.165 \\
\hline 4.6 & 0.520 & 10.5 & 3.935 \\
\hline 4.9 & 0.592 & 10.9 & 3.775 \\
\hline 5.1 & 0.680 & 11.6 & 4.415 \\
\hline 5.4 & 0.786 & 12.2 & 4.875 \\
\hline 5.7 & 0.914 & 12.7 & 5.263 \\
\hline 5.9 & 1.029 & 13.1 & 5.709 \\
\hline 6.1 & 1.068 & 13.6 & 6.194 \\
\hline 6.1 & 1.112 & 14.1 & 6.715 \\
\hline 6.3 & 1.242 & 14.7 & $6.764 ?$ \\
\hline 6.6 & 1.397 & 15.3 & 8.108 \\
\hline 6.9 & 1.553 & 16.3 & 9.348 \\
\hline 7.2 & 1.769 & 17.0 & 10.317 \\
\hline 7.5 & 1.940 & 18.3 & 12.120 \\
\hline 7.8 & 2.162 & 19.2 & 13.515 \\
\hline 8.1 & 2.362 & 20.7 & 15.013 \\
\hline 8.4 & 2.606 & 21.0 & 16.563 \\
\hline \multirow[t]{5}{*}{8.8} & 2.937 & 22.0 & 18.139 \\
\hline & & 23.2 & 29.577 \\
\hline & & 24.3 & 23.058 \\
\hline & & 25.6 & 25.425 \\
\hline & & 27.2 & 27.389 \\
\hline
\end{tabular}


Table 3-4. Specific Heat of NbTi/CU-Fiberglass Epoxy Coil Composite in Temperature Range 4.5 to $22.2 \mathrm{~K}$ (Run No. 2)

\begin{tabular}{|c|c|c|c|}
\hline $\begin{array}{l}\text { Temperature } \\
(\mathrm{K})\end{array}$ & $\begin{array}{l}\text { Specific Heat } \\
(\mathrm{J} / \mathrm{kg}-\mathrm{K})\end{array}$ & Temperature & $\begin{array}{l}\text { Specific Heat } \\
(\mathrm{J} / \mathrm{kg}-\mathrm{K})\end{array}$ \\
\hline 4.5 & 0.468 & 9.04 & 3.099 \\
\hline 4.6 & 0.521 & 9.15 & 3.104 \\
\hline 4.8 & 0.576 & 9.27 & 2.890 \\
\hline 4.9 & 0.612 & 9.39 & 2.783 \\
\hline 5.7 & 0.669 & 9.52 & 2.733 \\
\hline 5.3 & 0.744 & 9.66 & 2.876 \\
\hline 5.5 & 0.828 & 9.80 & 2.904 \\
\hline 5.8 & 0.958 & 9.94 & 3.081 \\
\hline 6.0 & 1.077 & 10.10 & 3.124 \\
\hline 6.3 & 1.202 & 10.27 & 3.244 \\
\hline 6.5 & 1.335 & 10.4 & 3.369 \\
\hline 6.7 & 1.473 & 10.6 & 3.533 \\
\hline 6.9 & 1.547 & 10.8 & 3.650 \\
\hline 7.1 & 1.652 & 11.0 & 3.848 \\
\hline 7.2 & 1.744 & 11.2 & 3.956 \\
\hline 7.3 & 1.821 & 11.4 & 4.219 \\
\hline 7.50 & 1.950 & 11.9 & 4.594 \\
\hline 7.56 & 1.952 & 12.2 & 4.851 \\
\hline 7.61 & 2.017 & 12.5 & 5.074 \\
\hline 7.71 & 2.097 & 12.9 & 5.417 \\
\hline 7.85 & 2.207 & 13.3 & 5.814 \\
\hline 7.99 & 2.281 & 13.7 & 6.188 \\
\hline 8.15 & 2.426 & 14.1 & 6.814 \\
\hline 8.30 & 2.526 & 14.6 & 7.057 \\
\hline 8.42 & 2.622 & 15.3 & 8.055 \\
\hline 8.50 & 2.649 & 17.1 & 10.227 \\
\hline 8.62 & 2.801 & 18.1 & 11.633 \\
\hline 8.72 & 2.835 & 19.3 & 13.372 \\
\hline 8.81 & 2.921 & 20.1 & 14.907 \\
\hline \multirow[t]{3}{*}{8.93} & 2.973 & 21.1 & 15.988 \\
\hline & & 22.2 & 19.123 \\
\hline & & & \\
\hline
\end{tabular}


Table 3-5. Specific Heat of Fiberglass-Epoxy Composite

\begin{tabular}{|c|c|c|c|}
\hline $\begin{array}{l}\text { Temperature } \\
\text { (K) }\end{array}$ & $\begin{array}{l}\text { Specific Heat } \\
(\mathrm{J} / \mathrm{kg}-\mathrm{K})\end{array}$ & $\begin{array}{l}\text { Temperature } \\
(\mathrm{K})\end{array}$ & $\begin{array}{c}\text { Specific Heat } \\
(\mathrm{J} / \mathrm{kg}-\mathrm{K})\end{array}$ \\
\hline 4.26 & 1.106 & 8.18 & 5.470 \\
\hline 4.35 & 1.144 & 8.39 & 5.898 \\
\hline 4.47 & 1.239 & 8.73 & 6.359 \\
\hline 4.62 & 1.340 & 9.21 & 7.412 \\
\hline 4.78 & 1.449 & 9.78 & 8.277 \\
\hline 4.94 & 1.598 & 10.57 & 9.482 \\
\hline 5.09 & 1.709 & 10.90 & 10.528 \\
\hline 5.25 & 1.874 & 11.27 & 11.307 \\
\hline 5.43 & 2.065 & 11.68 & 12.014 \\
\hline 5.68 & 2.288 & 12.24 & 13.357 \\
\hline 5.95 & 2.586 & 12.85 & 14.643 \\
\hline 6.19 & 2.852 & 13.44 & 15.724 \\
\hline 6.47 & 3.105 & 14.08 & 17.497 \\
\hline 6.71 & 3.402 & 14.68 & 19.302 \\
\hline 6.90 & 3.673 & 15.29 & 20.758 \\
\hline 7.09 & 3.971 & 16.39 & 24.001 \\
\hline 7.29 & 4.175 & 17.05 & 25.626 \\
\hline 7.52 & 4.467 & 18.01 & 28.134 \\
\hline 7.77 & 4.860 & 19.42 & 31.987 \\
\hline 8.03 & 5.293 & & \\
\hline
\end{tabular}


Table 3-6. Low-Temperature Specific heat Coefficients for Fiberglass-Epoxy Composite

$$
c=\alpha_{1}\left(\frac{T}{10}\right)+\alpha_{2}\left(\frac{T}{10}\right)^{2}+\alpha_{3}\left(\frac{T}{10}\right)^{3}+\alpha_{4}\left(\frac{T}{10}\right)^{4}
$$

\begin{tabular}{crr}
\hline \multirow{2}{*}{ Coefficient } & \multicolumn{2}{c}{ Value $\left(\mathrm{J}_{\mathrm{kgk}} \mathrm{kg}^{\mathrm{n}}\right.$} \\
\cline { 2 - 3 }$\alpha_{i}$ & $-1.81 \pm 0.14$ & $-1.81 \pm 0.05$ \\
$\alpha_{2}$ & $9.48 \pm 0.46$ & $9.48 \pm 0.15$ \\
$\alpha_{3}$ & $1.99 \pm 0.45$ & $1.99 \pm 0.15$ \\
$\alpha_{4}$ & $-1.05 \pm 0.13$ & $-1.05 \pm 0.04$ \\
\hline
\end{tabular}

Table 3-7. Low-Temperature Specific Heat of Fiberglass-Composite at Specific Temperatures

Temperature (K) Specific Heat $(\mathrm{J} / \mathrm{kg}$-deg)

4.0
5.0
10.0
15.0
20.0

$0.89 \pm 0.15$

$1.65 \pm 0.25$

8. \pm \pm 1.2

$20.0 \pm 2.2$

$33.4 \pm 5.1$ 
was determined to give the best fit to the experimental data. The values of the polynomial coefficients are listed in Table $3-6$. Because of the higher precision of the data above $10 \mathrm{~K}$, two sets of coefficients (and estimated uncertainties) are given.

As indicated in Table $3-6$, the quadratic term is dominant. Usually this term is absent in the expression for specific heat of a solid, as dictated by the physics of the three-dimensional model. The presence of a dominant quadratic term indicates the presence of two-dimensional, rather than three-dimensional, arrays of atomic oscillators. Whether the two dimensionality is due to the long, chain-like polymer molecules or to the reinforcement by the fiberglass is uncertain and could be the subject of further study.

The specific heat values of the fiberglass-epoxy at specified temperatures is given in Table 3-7 along with the estimated uncertainties.

\section{REFERENCES}

1. Fowlkes, C. W., Angerhofer, P. E., Newton, R. N., and Clark, A. F., "Characterization of a Superconducting Coil Composite," NBSIR 73-349, National Bureau of Standards, Boulder, CO (1973).

2. Childs, G. E., Ericks, L. J. and Powe11, R. L., "Thermal Conductivity of Solids at Room Temperature and Below," NBS Monograph 131, National Bureau of Standards, Boulder, CO (September 1973).

3. Clark, A. F., "Low Temperature Thermal Expansion of Some Metallic Alloys," Cryogenics $\underline{8}$, $282(1968)$.

4. McConne11, P. M., Daney, D. E., and Kirgis, J. B., "Thermoelastic Expansion and Creep of PET and PPMI Films and PET Fibers from 20 to $295 \mathrm{~K}, " \mathrm{~J}$. App1. Phys. 41, 5066-70 (Dec. 1970).

5. Hahn, T. A., "Thermal Expansion of Copper from 20 to $800 \mathrm{~K}$ - Standard Reference Material 736," J. App1. Phys. 41, 5096-5101 (Dec. 1970).

6. Corrucini, R. J. and Gniewek, J. J., "The Thermal Expansion of Technical Solids at Low Temperatures," National Bureau of Standards Monograph 29, U.S. Government Printing Office, Washington, D.C. (May 1961).

7. Jelinek, F. J. and Collings, E. W., "Low Temperature Thermal Expansion and Specific Heat Properties of Structural Materials," in: Materials Research for Superconducting Machinery - II, Clark, A. F., Reed, R. P., and van Reuth; E. C., Editors, ADA004586 (Octcber 1974). 


\section{ELASTIC PROPERTIES}

by W. F. Weston

\subsection{Introduction}

The elastic properties of composite materials are currently of considerable interest. Many composites, because they have high mechanical strength and a high modulus-to-density ratio, are useful as strong, lightweight structural materials. Some composites have been designed for use at low temperatures, for example as superconducting colls in generators or in motors. However, few elastic data are avallable for composite materials, particularly at low temperatures. Such data are useful as design parameters, and elastic constants are among the most accurately known fundamental physical properties of solids. Because composite materials are usually highly anisotropic, the determination of their elastic constants is much more difficult than for the more usual quasi-isotropic engineering materials.

Conventional methods of measuring the elastic properties of solids have been applied to composites with only limited success. These methods usually require relatively large specimens and considerable time and effort, especially if a complete set of elastic constants is required. ${ }^{{ }^{*}}$ These methods, as applied to composites, include vibrating ${ }^{2,3}$ or resonantbeam $^{4,5,6}$ tests, tensile or compressive tests, ${ }^{1,7,8}$ torsion tests, ${ }^{1,9}$ bending tests, ${ }^{1,10}$ and pressure tests $11,12,13$. Generally, the elastic constants measured by these methods are less accurate than those acquired by ultrasonic tests.

U1trasonic pulse techniques are limited by the necessity of using wavelengths that are large compared with fiber diameters and at the same time sufficiently small compared with the dimensions of the specimen so that true plane-wave conditions can exist. Since ultrasonic pulse methods are typically performed at megahertz frequencies, this condition cannot be met for many composites. If the wavelength is not larger than the fiber diameter, the wave is attenuated and scattered. U1trasonic pulse-echo methods have been applied to some metal-matrix composites with extremely small fibers. ${ }^{14,15,16}$ Even in these cases the pulse-echo trains are poor because of high attenuation and dispersion ${ }^{15}$. In fact, Achenbach and Herrman ${ }^{17}$ predicted large dispersive effects for shear waves propagated in the fiber direction, even when the wavelength is much larger than fiber diameters. Zimmer and Cost $^{18}$ verified this prediction by measuring sound velocities in a unfdirectional

* The references in each chapter are treated separately. 
glass-reinforced epoxy-fiber composite. For wavelength-to-fiber diameter ratios of roughly 50, dispersion increased some phase velocities by a factor of 2 . Elastic constants of some carbon-fiber-reinforced plastics have also been measured by ultrasonic pulse-echo techniques. $19,20,21$

The ultrasonic immersion technique, as described by Markham, ${ }^{22}$ is fast and accurate and offers the possibility of measuring all the elastic properties on one specimen. However, this technique is also limited by fiber size and by sample dimensions. 23,24 The choice of ultrasonic modes that can be propagated in a given direction in a specimen is also limited, and the technique is inapplicable to low-temperature measurements.

Zecca and Hay ${ }^{25}$ avoided the problems encountered with megahertz frequencies by using electrostatic transducers to generate and to detect resonant kilohertz frequencies of a metal-matrix composite. Electrostatic transducers, however, cannot be used with nonmetallic materials.

The most widely used ultrasonic technique, using kilohertz frequencies, is the resonant piezoelectric oscillator. This method is due principally to Quimby and associates. ${ }^{26}$ Quimby thoroughly analyzed the vibrations of solid rods driven by a piezoelectric crystal, and Balamuth ${ }^{27}$ first employed drivers and specimens of matching fundamental frequencies. Rose $^{28}$ extended this method to torsional oscillators. Basically, the piezoelectric properties of quartz are used to generate and to detect resonant frequencies of an oscillator consisting of one or two quartz crystals, a specimen, and perhaps a dummy rod, all cemented together. This method has been used to measure elastic moduli at both high ${ }^{29,30}$ and 1ow $27,28,30,31$ temperatures, and has worked well with both metals ${ }^{32,33}$ and non-metals. ${ }^{27-31}$ Thus, this technique is well understood both theoretically and practically, and it would seem to be well suited for testing composites. The long wavelengths used (typically on the order of centimeters in the kilohertz region) are much larger than fiber diameters, and, since the rechnique employs standing waves rather than traveling waves, specimen sizes can still be kept small.

In this section it is described how this resonance technique was used to measure the complete set of elastic compliances of a superconducting coil composite, which was essentially composed of an epoxy matrix and unidirectional copper-niobium-titanium fibers. The compliances were measured semi-continuously from 300 to $4 \mathrm{~K}$. Results are given also for 
an epoxy-fiberglass layered composite. The results generally agreed closely with the few existing static measurements on the same composite reported in section 2.3.

\subsection{Experimental Procedure}

\subsubsection{Resonant Piezoelectric Oscillator}

The three-component resonant piezoelectric oscillator technique was originally described by $\mathrm{Marx}^{34}$, and was discussed in detail by Fine ${ }^{35}$. The technique consists of bonding quartz-driver and quartz-gauge piezoelectric crystals to a specimen to produce and to detect a standing longitudinal (or torsional) resonant wave. Each component's length is adjusted so that its resonant frequency is closely matched to that of the other components. The system is then driven by the driver transducer at its resonant frequency, which is monitored by the gauge crystal.

Longitudinal waves are excited by $\alpha$-quartz bars of square cross section, excited into longitudinal vibration by an ac signal applied to full-length, adherent electrodes (see Fig. 4-1). The driver quartz was fully gold plated on two parallel sides (z-faces) with a shallow notch cut in the center of each electrode. Fine, gold-plated tungsten wires, seated in these notches, served to suspend the resonator assembly at displacement nodes and provided the necessary electrical contact. The gauge quartz was gold-plated only over the center third of the two parallel faces and electrical contact was made through fine wires adhered into the notches with silver conducting paint. The electric axes of the driver and the gauge crystals were perpendicular to reduce electrical pickup.

The torsional quartz crystals were circular cylinders with their length in the $\mathrm{x}$-direction and with four gold-plated electrodes running the length of the crystal. ${ }^{36}$ Opposite pairs of electrodes were electrically connected. The driver quartz was suspended vertically by means of fine nylon threads attached with varnish to opposite sides of the quartz near a displacement node of vibration.

The quartz crystals were bonded together with a semi-permanent adhesive, Eastman 910*. The specimens were right-circular cylinders and were also bonded to the gauge quartz with the same adhesive. For some torsional experiments, this cement occasionally failed at low temperatures; such experiments were repeated until successful. Other materials such as vacuum grease and epoxy resin have been used as low-temperature bonding agents. ${ }^{37}$

* The use in this paper of trade names of specific products is essential to a proper understanding of the work presented. Their use in no way implies any approval, endorsement, or recommendation by NBS. 


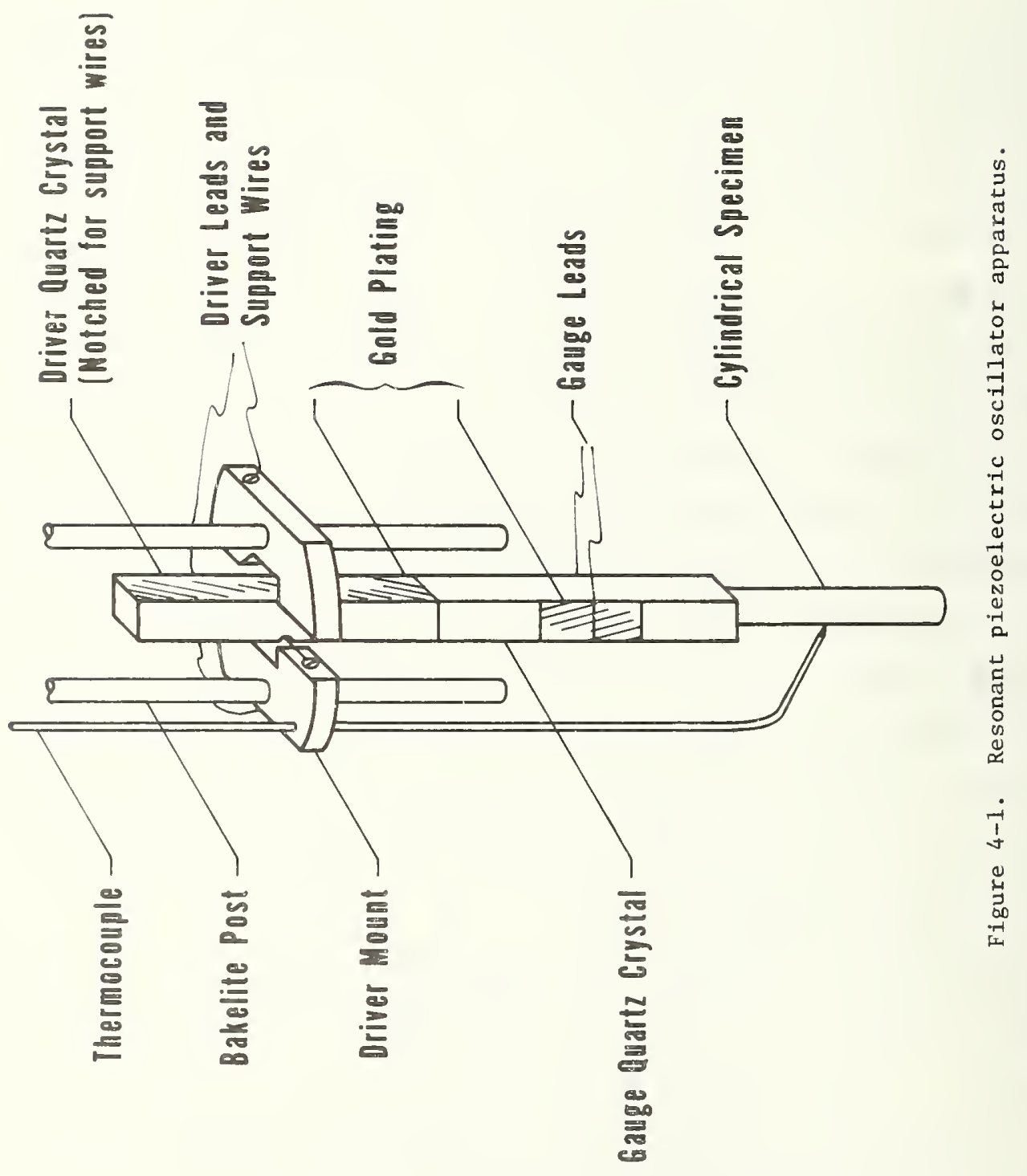




\subsubsection{Electronics}

Most workers who have used the piezoelectric oscillator technique have employed electronics similar to those described by Marx ${ }^{34}$. This system requires manual adjustment of the input frequency to the driver crystal to keep the oscillator at resonance. A less time-consuming and more accurate system is the regenerative system designed by Gerk $^{38}$ and slightly modified by Johnson ${ }^{39}$.

This system consists primarily of a closed loop containing an amplifier and the driver-gauge combination of piezoelectric crystals, which act as a resonant element, with a feedback circuit to control the gain of the amplifier. This system is based on the principles of an oscillator; that is, the output of an amplifier is fed into the input through a frequency-selective network (the quartz crystals). The phase shifts are corrected with a variable phase shifter. A more complete description of the system was given by Gerk. ${ }^{38}$ This system locks onto the resonant frequency and automatically stays at the resonant frequency if this frequency changes, due to a change of the specimen's environment, for example.

\subsubsection{Apparatus}

The longitudinal oscillator apparatus is illustrated in Figure 4-1. The driver-quartz crystal was suspended by $0.008 \mathrm{~cm}$ gold-plated tungsten wires. The driver mounts, which were aluminum, were removed during the torsional measurements, since the torsional driver crystal was suspended between the bakelite posts by nylon threads. The specimens were cylindrical for all measurements. The chromel-constantan thermocouple was placed near, but not touching, the specimen. The entire apparatus was sealed in a thin-walled stainlesssteel can. The can was partially evacuated, placed in the ullage of a hellum dewar and lowered stepwise to achieve cooling.

The quartz crystals used had resonant frequencies of 60 and $100 \mathrm{kHz}$. Most of the measurements were done with the $60 \mathrm{kHz}$ quartz crystals (3/16 in $(0.48 \mathrm{~cm})$ square for longitudinal, and $3 / 16 \mathrm{in}(0.48 \mathrm{~cm})$ diameter for torsional), but some were done with the $100 \mathrm{kHz}$ crystals (1/8 in $(0.32 \mathrm{~cm})$ diameter). The mass of each driver-gauge combination was noted and the resonant frequencies were monitored from 300 to $4 \mathrm{~K}$. The length of each specimen was determined such that its resonant frequency for the entire temperature range was within approximately $5 \%$ of the frequency of the oscillator assembly although in some cases the specimen frequency differed by as much as $10 \%$. The mass, length, and diameter of each 
specimen were noted and its mass density was determined by Archimedes' method using distilled water as a standard. The specimen was then cemented to the quartz crystals and the resonant frequency of the oscillator was monitored from 300 to $4 \mathrm{~K}$. No thermal contraction corrections were made; for the coil composite described below this introduces a maximum error (over a $300 \mathrm{~K}$ range) of about $0.5 \% .40$ Maximum uncertaintites in the frequency measurements are estimated to be about one part in $10^{5}$.

\subsubsection{Specimens}

The superconducting coil composite is fully described in reference 40. Basically, the coil is composed of a copper-stabilized niobium-titanium wire $(0.56 \mathrm{~mm}$ by $0.72 \mathrm{~mm}$ cross section) coil impregnated with epoxy. The wire layers are separated by layers of dry fiberglass cloth (0.1 mm thick). The copper-to-superconductor volume ratio of the wire is 1.8 to 1 . The wire-to-epoxy cross-sectional area fraction is about 3 to 1 . The coil dimensions were approximately $19 \mathrm{~cm} \mathrm{I.D.,} 24 \mathrm{~cm} \mathrm{O.D.,} \mathrm{and} 10 \mathrm{~cm}$ long.

Coordinate axes were chosen to coincide with the specimen axes. The longitudinal axis (1-axis) coincides with the axis of the wire, which was assumed to have zero helix angle. The 2- and 3-axes lie along the radius and coil axes, respectively, and form an orthogonal set with the 1-axis. The symmetry of the coil (orthorhombic) requires nine elastic constants to characterize the material. By assuming the material to be transversely isotropic, and by neglecting the effects of curvature, the number of independent elastic constants is reduced to five. An orthotropic body with transverse isotropy has the same symetry as close-packed hexagonal crystals such as magnesium and zinc. Justification for equating the 2- and 3-axes is given in reference 40, and further experimental data show that the effect of curvature on the moduli is minor. ${ }^{41}$

The masses, lengths, and angles between the specimen axis and the 1-axis are given in Table 4-1. The density of the coil composite was found to be $6.000 \mathrm{gm} / \mathrm{cm} .{ }^{3}$

Besides the superconducting coil, some specimens composed of fiberglass cloth and epoxy were made available. The fiberglass-to-epoxy volume ratio in these samples was approximately the same as in the coll composite. Measurements were made on two specimens of this material. Dimensions and orientations of these specimens are also reported in Table 4-1, with the angle $\theta$ corresponding to the angle between the specimen axis and the direction direction parallel to the layers of fiberglass cloth. The density of this naterial was found to be $1.931 \mathrm{gm} / \mathrm{cm}^{3}$. This material can also be considered transversely 
Table 4-1. Dimensions and orientation of specimens

\begin{tabular}{lcccc}
\hline $\begin{array}{c}\text { Specimen } \\
\text { Number }\end{array}$ & $\begin{array}{c}\text { Diameter } \\
(\mathrm{cm})\end{array}$ & $\begin{array}{c}\text { Mass } \\
(\mathrm{gm})\end{array}$ & $\begin{array}{c}\text { Length } \\
(\mathrm{cm})\end{array}$ & $\begin{array}{c}\theta \\
\text { (degrees) }\end{array}$ \\
\hline 1 & Superconducting & Coil & Composite & Specimens \\
2 & 0.3175 & 0.540 & 1.1128 & 90 \\
3 & 0.3175 & 0.925 & 1.9439 & 0 \\
4 & 0.4732 & 1.081 & 1.0818 & 0 \\
5 & 0.4801 & 1.248 & 1.1481 & 90 \\
6 & 0.4763 & 1.874 & 1.7600 & 60 \\
1 & 0.4702 & 1.327 & 1.2555 & 60 \\
2 & Epoxy-Fiberglass & Composite & Specimens & 0 \\
\hline
\end{tabular}


isotropic. Thus, five constants are again needed to completely characterize the material elastically. Cylindrical specimens of only one orientation were available; thus, only two elastic constants of the fiberglass-epoxy matrix material were determined.

\subsection{Results}

The directly measured quantity in these measurements is $f_{0}$, the frequency of the three-component piezoelectric resonator. For longitudinal resonance the frequency of the specimen $\left(f_{s}\right.$ ) can be found from $f_{o}$, the frequency of the driver-gauge quartz assembly ( $\left.f_{q}\right)$, the mass of the specimen $\left(m_{s}\right)$, and the mass of the quartz $\left(m_{q}\right)^{42}$ :

$$
f_{s}^{2}=f_{o}^{2}+\left(f_{o}^{2}-f_{q}^{2}\right) m_{q} / m_{s}
$$

This formula is more exact than the approximation

$$
\mathrm{f}_{\mathrm{s}}=\mathrm{f}_{\mathrm{o}}+\left(\mathrm{f}_{\mathrm{o}}-\mathrm{f}_{\mathrm{q}}\right) \mathrm{m}_{\mathrm{q}} / \mathrm{m}_{\mathrm{s}} \text {, }
$$

which is usually quoted and used for resonant oscillators. 34

The formula for the torsional oscillator is somewhat more complicated since the moments of inertia (rather than the masses) of the components are involved: ${ }^{36}$

$$
\mathrm{f}_{\mathrm{s}}^{2}=\mathrm{f}_{\mathrm{o}}^{2}+\left(\mathrm{f}_{\mathrm{o}}^{2}-\mathrm{f}_{\mathrm{q}}^{2}\right) \mathrm{m}_{\mathrm{q}} \mathrm{r}_{\mathrm{q}}^{2} /\left(\mathrm{m}_{\mathrm{s}} \mathrm{r}_{\mathrm{s}}^{2}\right)
$$

Here, $\mathrm{r}_{\mathrm{q}}$ is the radius of the quartz and $\mathrm{r}_{\mathrm{s}}$ is the radius of the specimen.

The Young's modulus, E, or shear modulus, G, for the particular orientation of the specimen is given by:

$$
E \text { or } G=4 \mathrm{~L}^{2} \mathrm{f}_{\mathrm{s}}^{2} \mathrm{\rho} \text {. }
$$

$E$ is found from the longitudinal-mode fundamental frequency and $G$ is found from the torsional-mode fundamental frequency. In Eq. 4-4, L is the specimen length and $\rho$ is the mass density.

Thus, the measured quantities are the Young's and shear moduli, which are directly related to the elastic compliances, $S_{i j}$. Elastic compliances rather than elastic stiffnesses, $C_{i j}$, are measured because of the fundamental difference between the behavior of high-frequency and low-frequency elastic waves. In the case of a high-frequency wave (negahertz and higher), there is insufficient time in a cycle for the material to relax 
and to relieve the stresses due to the wave. Thus, for high-frequency measurements, the strains are relatively simple but the stresses are complicated and the elastic stiffnesses are the constants used in solving the elastic-wave equation. For low-frequency measurements, the reverse is true, and the elastic compliances are the constants of interest. However, for low frequencies, if the wavelength is not much larger than the sample dimensions, a correction for the Poisson contraction (or lateral motion) must be applied to Young's modulus. ${ }^{35}$ When the length-to-diameter ratio is 10 to 1 , this correction amounts to about one part in a thousand.

The value of Young's modulus for an arbitrary direction in a specimen with transverse isotropy, with the 1-axis the unique axis, is given in terms of the elastic compliances by $^{43}$

$$
\frac{1}{\mathrm{E}}=\mathrm{s}_{33} \sin ^{4} \theta+\mathrm{s}_{11} \cos ^{4} \theta+\left(2 \mathrm{~s}_{12}+\mathrm{s}_{55}\right) \sin ^{2} \theta \cos ^{2} \theta
$$

where $\theta$ is the angle between the specimen axis and the unique axis. Simflarly, the shear modulus is

$$
\frac{1}{G}=S_{55}+1 / 2\left(S_{33}-S_{23}-1 / 2 S_{55}\right) \sin ^{2} \theta+2\left(S_{33}+s_{11}-2 S_{12}-s_{55}\right) \sin ^{2} \theta \cos ^{2} \theta \cdot(4-6)
$$

\subsubsection{Superconducting Coil Composite}

Measurements made on the coll composite are 1isted in Table 4-2. Again, $\theta$ is the angle between the specimen axis and the 1-axis (longitudinal axis). The mode specifies either longitudinal or torsional oscillations. The subscripts on $E$ and $G$ designate the direction of the force and the plane on which the force is applied, respectively. The compliances $\mathrm{S}_{33}, \mathrm{~S}_{11}$, and $\mathrm{S}_{55}$ are found directly from experiments 1, 2, and 3, respectively. From experiment $4, \mathrm{~S}_{23}$ is found using the relation $2\left(\mathrm{~S}_{33}-\mathrm{S}_{23}\right)=\mathrm{S}_{44}$, and the value of $\mathrm{S}_{55}$ from experiment 3. Either specimen 5 or 6 can be used to determine $\mathrm{S}_{12}$. This compliance must be determined from a measurement on a specimen with its axis at an angle to the longitudinal direction. As can be seen from Table 4-2, however, the Young's or shear modulus measured on specimens with $0^{\circ}<\theta<90^{\circ}$, is only partly dependent on $\mathrm{S}_{12}{ }^{\circ}$ Thus $\mathrm{S}_{12}$ will be the least accurately determined compliance. The measurement of this compliance corresponds to the measurement of the elastic stiffness $C_{13}$ by megahertz ultrasonic techniques. 
Table 4-2. Experiments performed on superconducting coil composite

\begin{tabular}{|c|c|c|c|c|}
\hline Exp. No. & Spec. No. & Mode & $\theta$ & Measured Quantity \\
\hline 1 & 1 & $\mathrm{~L}$ & 90 & $\mathrm{E}_{22}=\frac{1}{\mathrm{~S}_{33}}$ \\
\hline 2 & 2 & L & 0 & $\mathrm{E}_{11}=\frac{1}{\mathrm{~S}_{11}}$ \\
\hline 3 & 3 & $\mathrm{~T}$ & 0 & $G_{13}=\frac{1}{S_{55}}$ \\
\hline 4 & 4 & $\mathrm{~T}$ & 90 & $G^{\prime}=\frac{2}{S_{55+S_{44}}}$ \\
\hline 5 & 5 & L & 60 & $E_{\theta=60^{\circ}}=\frac{16}{9 S_{33}+S_{11}+6 S_{12}+3 S_{55}}$ \\
\hline 6 & 6 & $\mathrm{~T}$ & 60 & $G_{\theta=60^{\circ}}=\frac{8}{3 S_{33}+3 S_{11}-6 S_{12}+2 S_{5}}$ \\
\hline
\end{tabular}


Zimmer and Cost $^{17}$ encountered difficulty in measuring $C_{13}$ for a composite material and their estimated uncertainty is this elastic constant (100\%) was ascribed to dispersion as well as to the relatively high inaccuracy.

The experimental data are presented in Figures 4-2,7. Temperature dependences of these moduli were fitted to a theoretical relationship suggested by Varnshni 44

$$
C=C^{0}-\frac{s}{e^{t / T}-1}
$$

where $C^{\circ}, s$, and $t$ are adjustable parameters and $T$ is temperature. The value of $C$ at $\mathrm{T}=0 \mathrm{~K}$ is $\mathrm{C}^{\circ}$, and $-\mathrm{s} / \mathrm{t}$ is the high-temperature limit of the temperature derivative $\mathrm{dC} / \mathrm{dT}$. By invoking an Einstein oscillator model of solids, it can be shown (in the absence of electronic effects) that $t$ is the Einstein characteristic temperature. This function has been used successfully to represent the temperature dependences of metals, ${ }^{44}$ ionic solids, 44 and several alloys. ${ }^{45}$ The parameters $c^{\circ}, s$, and $t$ are given in Table 4-3. The overall fit of this function to these data is not nearly as good as for other materials. In general, the Varshni function is flatter at low temperatures than these data, giving lower curve values at $0 \mathrm{~K}$. It also has smaller temperature derivatives, giving larger curve values near room temperature. The goodness of the fit is also listed in Table 4-3 as the average percentage difference between the curve values and the experimental values. These percentage differences are larger than was found for alloy data. ${ }^{45}$ One set of data, $E_{11}$, could not be fitted to the Varshni function because of its concave-upwards curvature. A smooth curve was drawn through the data points and the actual point values were used in calculations.

The shear modulus corresponding to shearing the axial planes in the radial direction $G_{23}$, or vice-versa is shown in Figure 4-8. This modulus was calculated from the curve values of $G_{13}$ and $G^{\prime}$, since $G_{23}=1 / S_{44}$.

Because of the difficulty in machining the specimens to small radif, the ratios of lengths to diameters were in some cases on the order of four or five to one. The estimated correction to the Young's moduli in these cases is still only about one percent.

The elastic compliances as functions of temperature are given in Table 4-4. The compliances $\mathrm{S}_{32}, \mathrm{~S}_{11}$, and $\mathrm{S}_{55}$ are just reciprocals of $\mathrm{E}_{22}, \mathrm{E}_{11}$, and $\mathrm{G}_{13}$, as mentioned previous1y. The error in the directly measured moduli is estimated to be about $1 \%$, thus these compliances should be accurate to $1 \%$, also. The compliance $\mathrm{s}_{23}$ is found from 


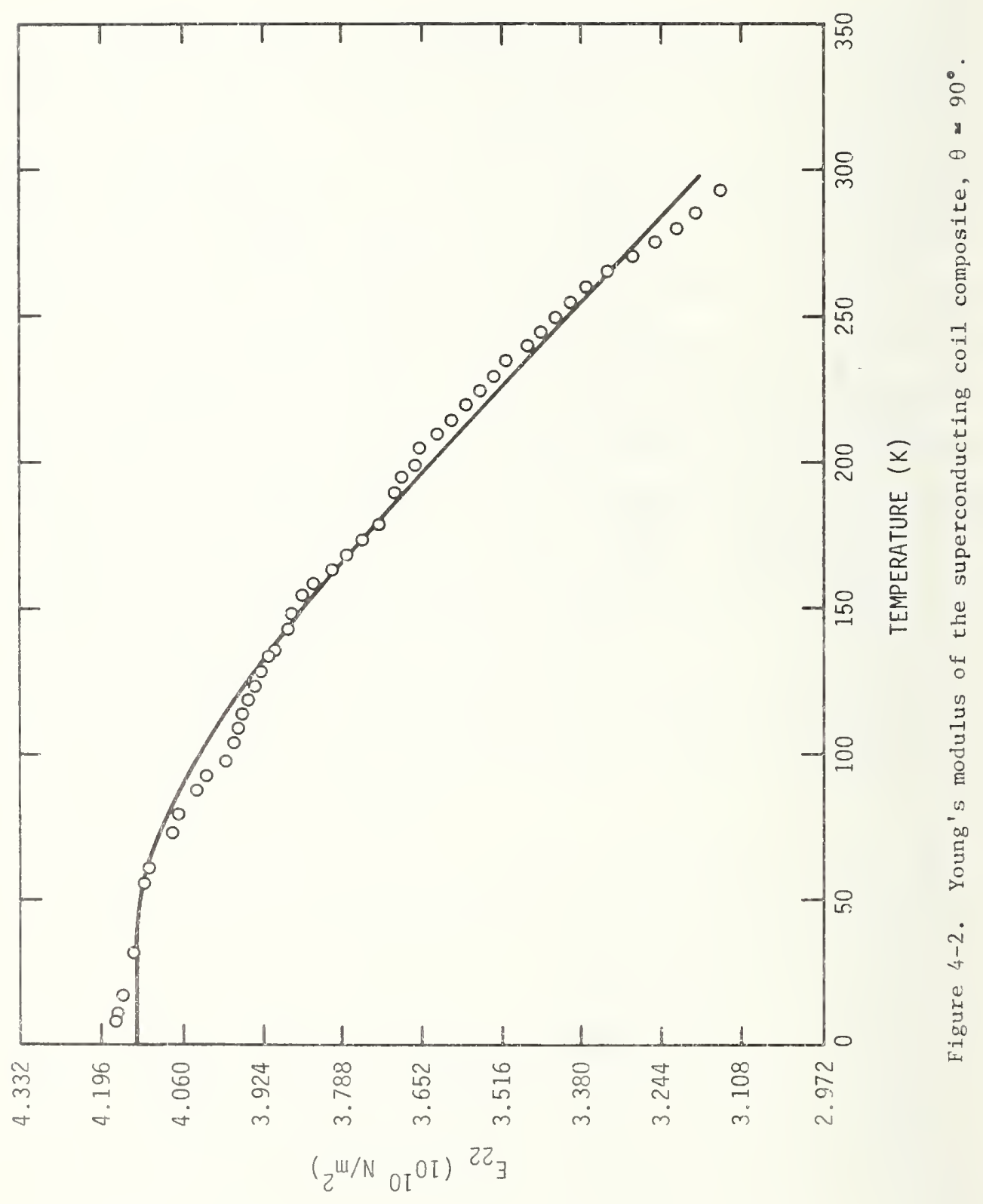




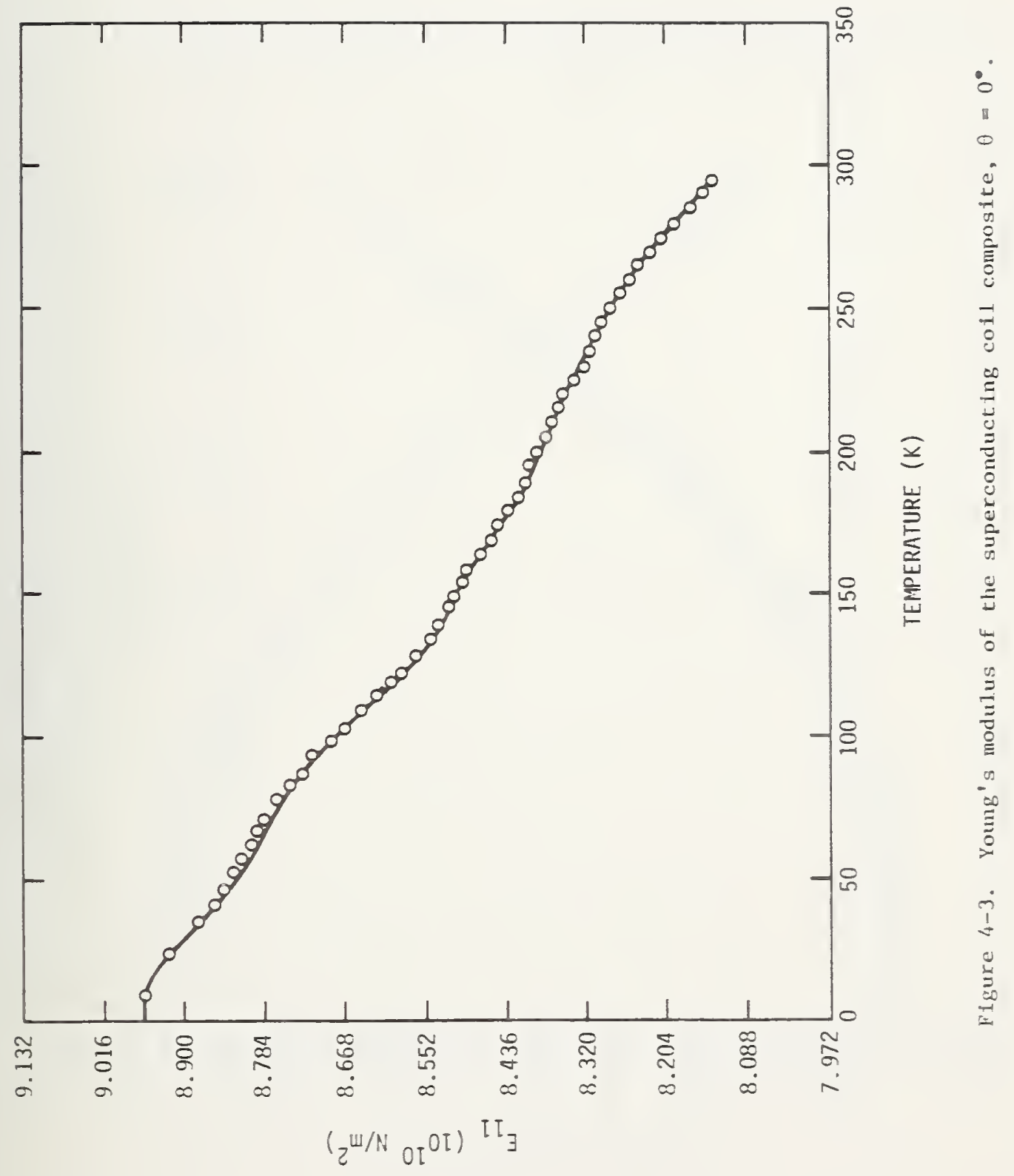




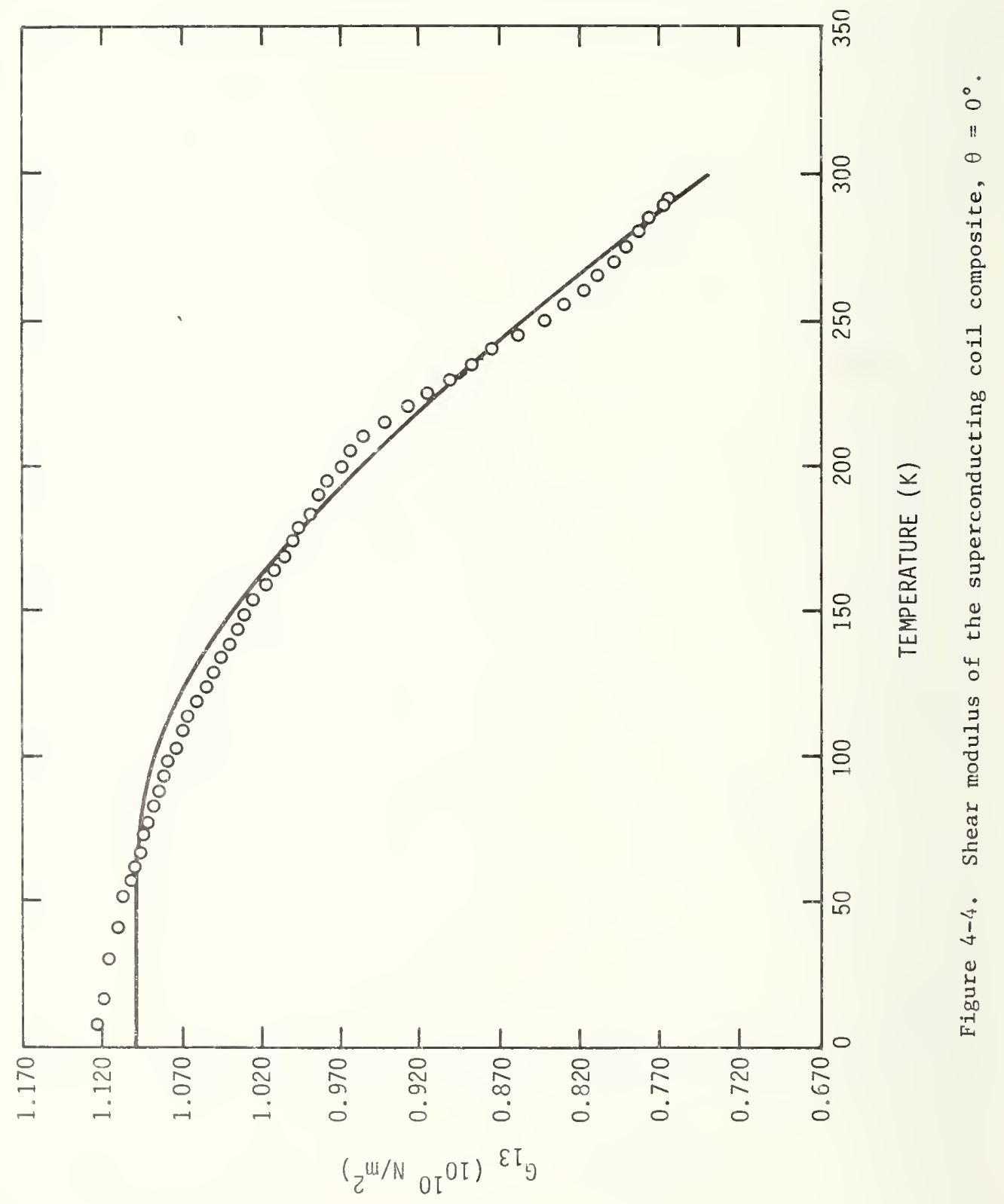




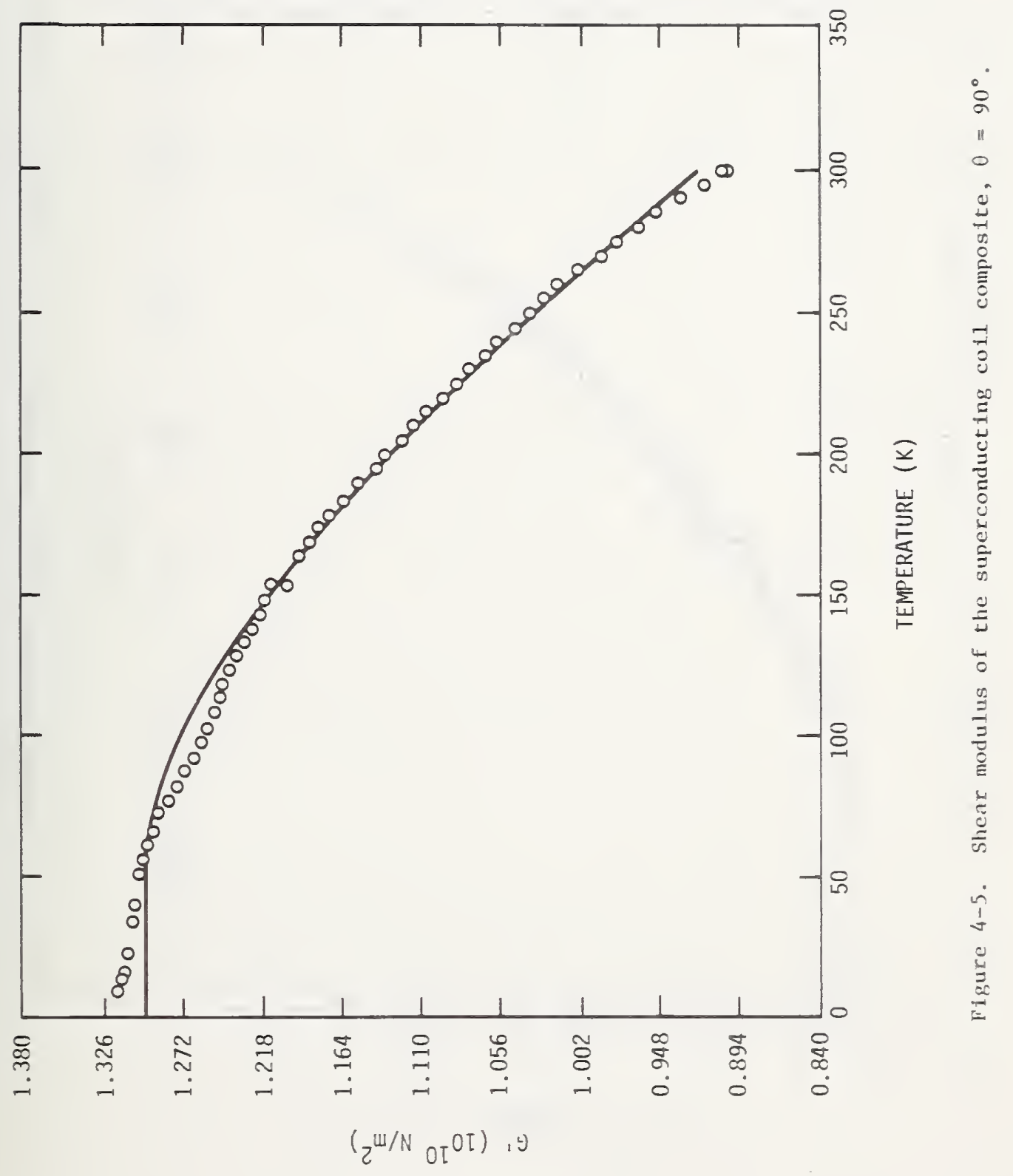




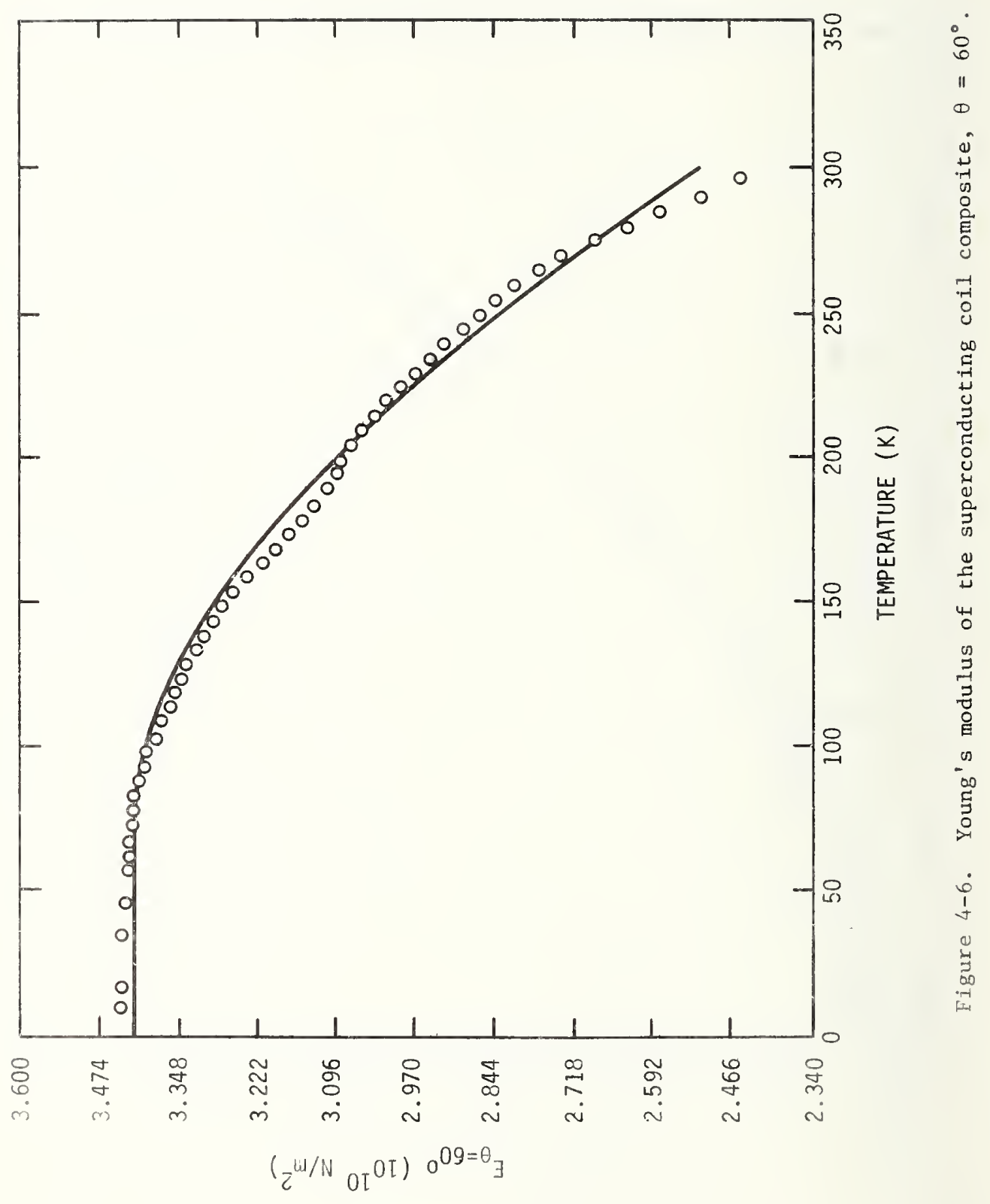




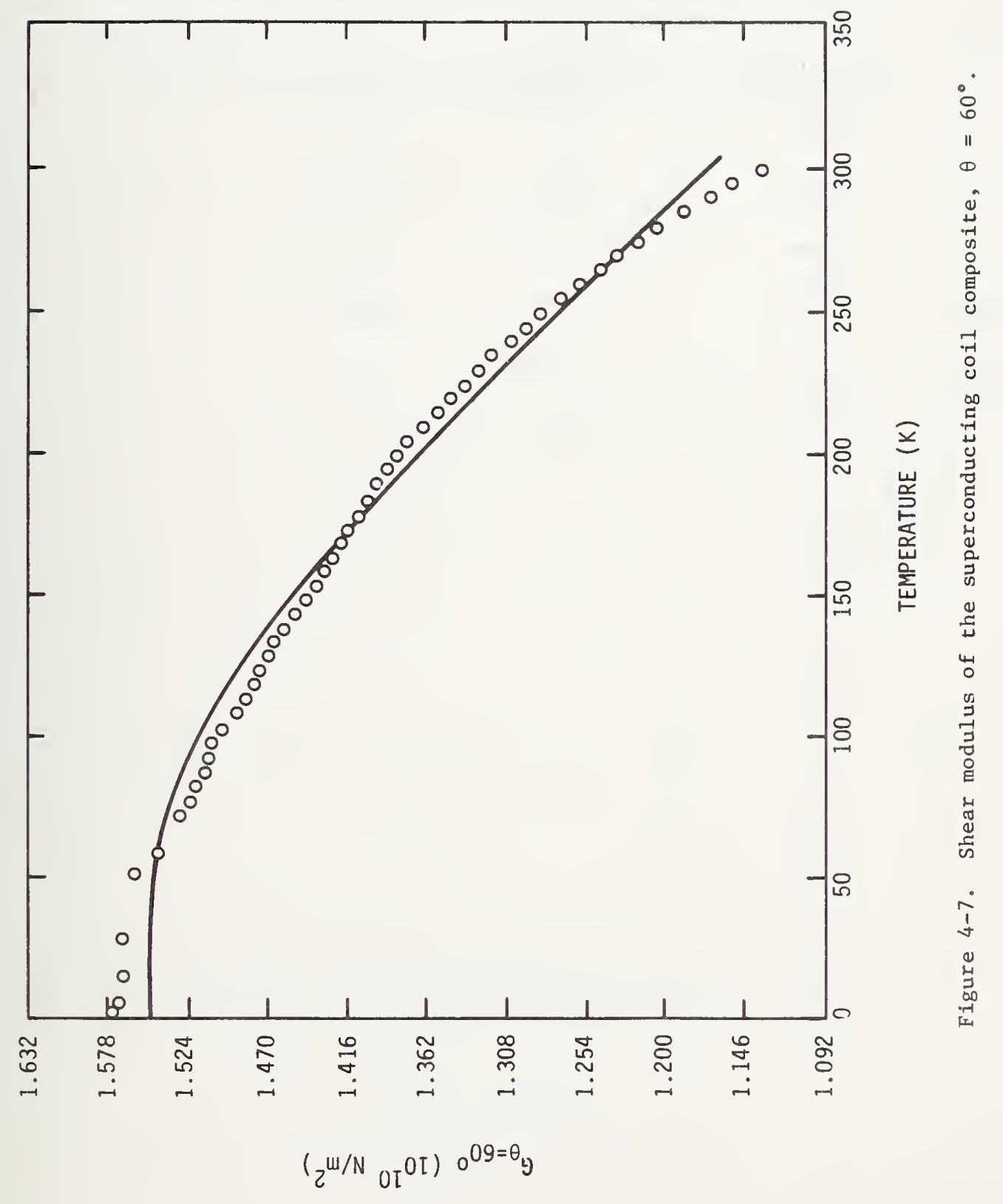


Table 4-3. Parameters in equation 7

\begin{tabular}{lcccc}
\hline Mode & $\begin{array}{c}\mathrm{C}^{\mathrm{O}} \\
\left(10^{\left.10 \mathrm{~N} / \mathrm{m}^{2}\right)}\right.\end{array}$ & $\begin{array}{c}\mathrm{s} \\
\left(10^{10} \mathrm{~N} / \mathrm{m}^{2}\right)\end{array}$ & $\begin{array}{c}\mathrm{t} \\
(\mathrm{K})\end{array}$ & Avg. \% Diff. \\
\hline $\mathrm{E}_{22}$ & 4.137 & 1.373 & 264.5 & 0.63 \\
$\mathrm{G}_{13}$ & 1.099 & 1.352 & 469.0 & 0.89 \\
$\mathrm{G}^{\prime}$ & 1.298 & 0.930 & 372.8 & 0.67 \\
$\mathrm{E}_{\theta=60^{\circ}}$ & 3.419 & 4.431 & 533.5 & 0.74 \\
$\mathrm{G}_{\theta=60^{\circ}}$ & 1.550 & 0.724 & 320.7 & 0.94 \\
\hline
\end{tabular}




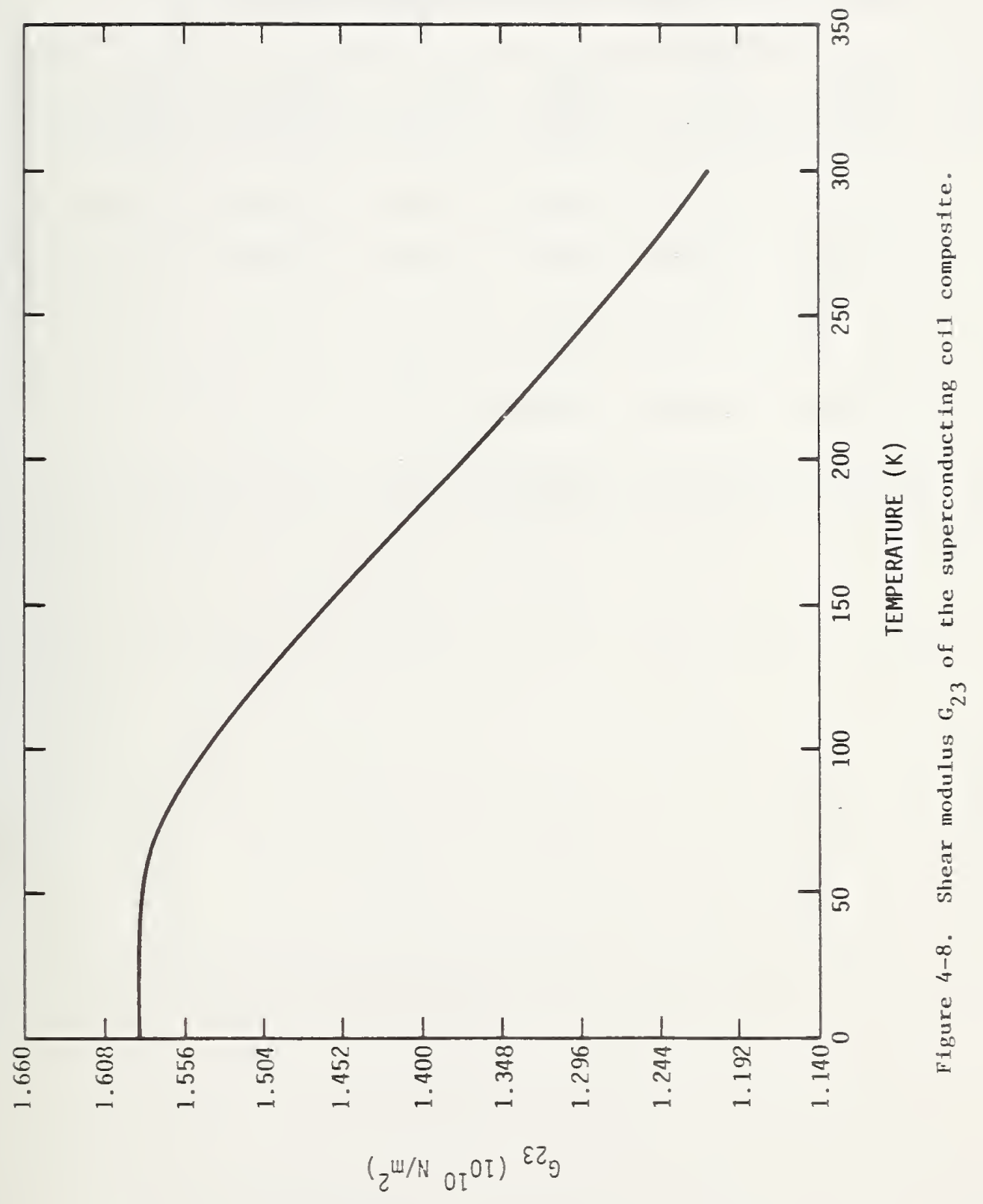


Table 4-4. Elastic compliances of the superconducting coil composite at selected temperatures $\left(10^{-10} \mathrm{~m}^{2} / \mathrm{N}\right)$

\begin{tabular}{ccccccc}
\hline $\mathrm{T}(\mathrm{K})$ & $\mathrm{S}_{11}$ & $\mathrm{~S}_{12}$ & $\mathrm{~S}_{23}$ & $\mathrm{~S}_{33}$ & $\mathrm{~s}_{44}$ & $\mathrm{~S}_{55}$ \\
\hline 0 & 0.112 & -0.061 & -0.074 & 0.242 & 0.631 & 0.910 \\
50 & 0.113 & -0.061 & -0.074 & 0.242 & 0.632 & 0.910 \\
100 & 0.115 & -0.066 & -0.076 & 0.248 & 0.648 & 0.920 \\
150 & 0.118 & -0.073 & -0.083 & 0.260 & 0.684 & 0.964 \\
200 & 0.119 & -0.079 & -0.089 & 0.275 & 0.728 & 1.046 \\
250 & 0.121 & -0.082 & -0.094 & 0.294 & 0.776 & 1.170 \\
300 & 0.123 & -0.084 & -0.096 & 0.316 & 0.823 & 1.350 \\
\hline
\end{tabular}


Table 4.5. Elastic stiffnesses of the superconducting coil composite at selected temperatures $\left(10^{10} \mathrm{~N} / \mathrm{m}^{2}\right)$

\begin{tabular}{rllllll}
\hline$T(K)$ & $C_{11}$ & $C_{12}$ & $C_{23}$ & $C_{33}$ & $C_{44}$ & $C_{55}$ \\
\hline 0 & 10.59 & 2.39 & 1.94 & 5.10 & 1.58 & 1.10 \\
50 & 10.46 & 2.39 & 1.93 & 5.10 & 1.58 & 1.10 \\
100 & 10.27 & 2.34 & 1.90 & 4.99 & 1.54 & 1.09 \\
150 & 10.05 & 2.27 & 1.87 & 4.80 & 1.46 & 1.04 \\
200 & 9.86 & 2.15 & 1.79 & 4.54 & 1.37 & 0.96 \\
250 & 9.64 & 1.99 & 1.63 & 4.21 & 1.29 & 0.85 \\
300 & 9.35 & 1.77 & 1.39 & 3.82 & 1.22 & 0.74 \\
\hline
\end{tabular}


combining the results of three measurements. Thus, $\mathrm{S}_{33}$ may be less accurately known. It was found that in solving for $\mathrm{S}_{12}$ from either $\mathrm{Eq} .5$ or 6 , a $1 \%$ error in the measured modulus led to a $10-20 \%$ error in $\mathrm{S}_{12}$. Thus, the error associated with $\mathrm{S}_{12}$ is considerable, perhaps as much as $50 \%$. The values reported for $\mathrm{S}_{12}$ are the average of the values found from experiments 5 and 6 . As expected, the material was found to be highly anisotropic with $\mathrm{S}_{33} \approx 2 \mathrm{~S}_{11}$.

\subsubsection{Epoxy-Fiberglass Composite}

Measurements were also made on the epoxy-fiberglass specimens listed in Table 4-1. These specimens were oriented with the layers of fiberglass cloth running the length of the specimen; these were the only orientations available. The Young's modulus and shear modulus are shown in Figures 4-9 and 4-10. The shear modulus was fitted to the Varshni function with $\mathrm{C}^{\circ}=0.885 \times 10^{10} \mathrm{~N} / \mathrm{m}^{2}, \mathrm{~S}=0.227 \times 10^{10} \mathrm{~N} / \mathrm{m}^{2}$, and $\mathrm{T}=193.8 \mathrm{~K}$. The average percentage difference between the curve value and the measured value was $0.28 \%$. A smooth curve was drawn through the Young's modulus data, which could not be fitted to the Varshni function because of the low-temperature maximum.

\subsection{Discussion}

Some elastic data, taken by conventional static methods, are available in Section 2.3 for the superconducting coil composite and the epoxy-fiberglass composite. These data generally compare very favorably with the resonance data. See Table 4-6. For example, resonance values of $\mathrm{E}_{22}$ at room temperature and $4 \mathrm{~K}$ are $12 \%$ and $1 \%$ larger than the static data taken at these temperatures. The inaccuracy of the static values is given as about 10\%. Also, resonance values of $E_{11}$ compare to within $7-5 \%$ of the static data, and resonance values of $G_{13}$ agree within $3-9 \%$ of the static values.

Other elastic constants of interest include the Poisson ratios:

$$
v_{23}=\mathrm{s}_{23} / \mathrm{s}_{33}
$$

and

$$
v_{21}=-\mathrm{s}_{12} / \mathrm{s}_{11}
$$

where $v_{i j}$ represents the negative ratio of strain in the j-direction to strain in the i-direction.

The value of $v_{23}$ at room temperature is 0.304 . The reported ${ }^{40}$ static value is 0.335 , with the inaccuracy again believed to be about $10 \%$. Thus, this result gives added confidence to the resonance value of $\mathrm{S}_{23}$. However, the Poisson ratio $\nu_{21}$, involves the 


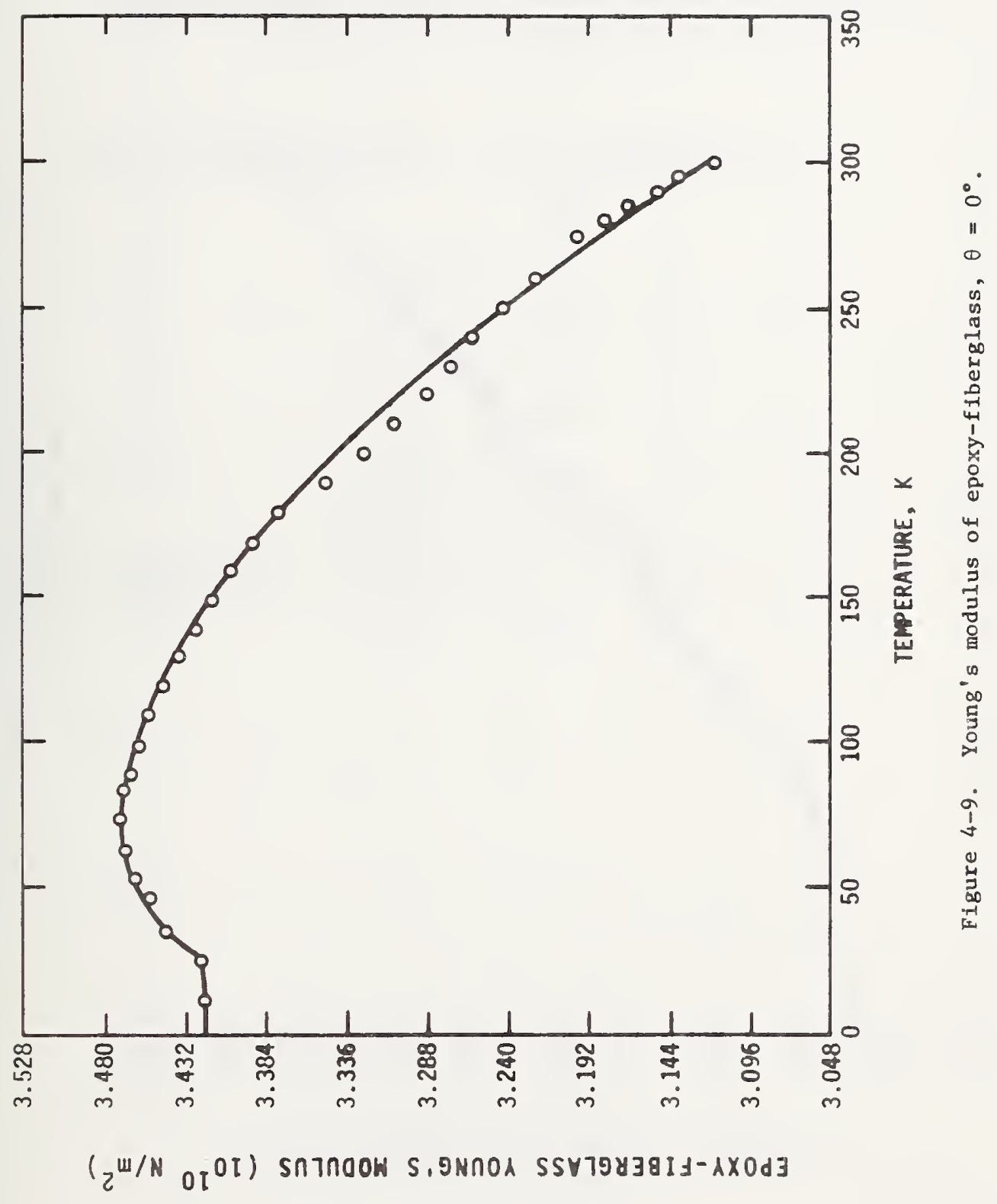




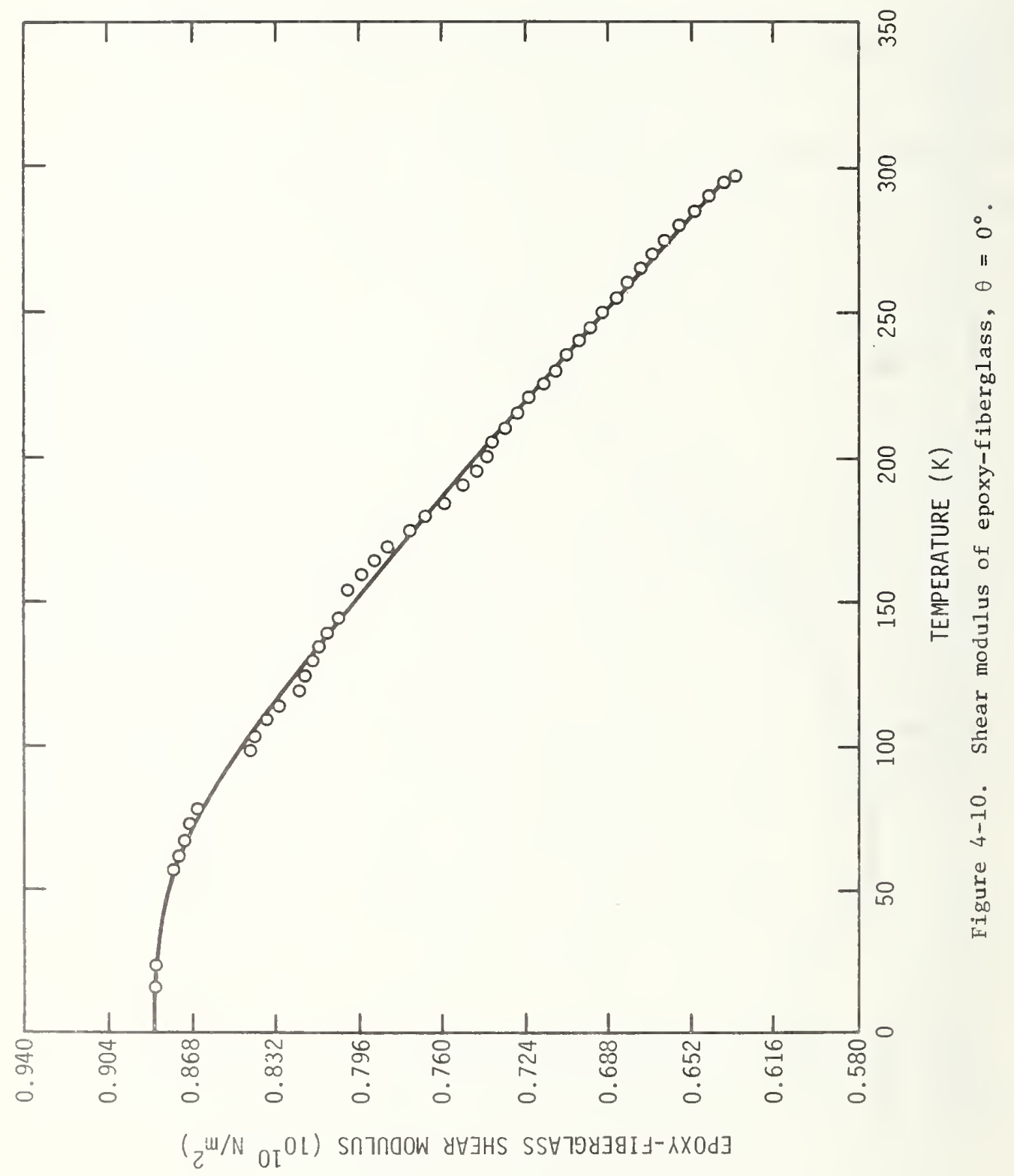


Table 4-6. Comparison of static and acoustic measurements of elastic constants (units of $10^{-10} \mathrm{~N} / \mathrm{m}^{2}$ ).

\begin{tabular}{|c|c|c|c|c|}
\hline \multicolumn{2}{|c|}{ Elastic Constant } & \multicolumn{3}{|c|}{ Temperature $(\mathrm{K})$} \\
\hline & & 293 & 77 & $\underline{4}$ \\
\hline \multirow[t]{2}{*}{$E_{11}$} & acoustic & 8.14 & 8.77 & 8.96 \\
\hline & static & 7.58 & 8.08 & 8.53 \\
\hline \multirow[t]{2}{*}{$E_{22}$} & acoustic & 3.14 & 4.07 & 4.17 \\
\hline & static & 2.76 & 3.79 & 4.12 \\
\hline \multirow[t]{2}{*}{$G_{13}$} & acoustic & 0.76 & 1.09 & 1.12 \\
\hline & static & 0.74 & 1.16 & 1.22 \\
\hline \multirow{2}{*}{$\begin{array}{l}E_{\text {fiberglass }} \\
\text { epoxy }\end{array}$} & acoustic & 3.14 & 3.47 & 3.42 \\
\hline & static & 2.80 & 3.33 & 3.56 \\
\hline
\end{tabular}


compliance $\mathrm{S}_{12}$, which has already been noted to be much more inaccurate than the other four compliances. In fact, if the room-temperature values of $\mathrm{s}_{12}$ and $\mathrm{s}_{11}$ are used, $\nu_{21}=0.683$, whereas the static value is $0.333 .^{40}$ Thus, the value of $\mathrm{s}_{12}$ is highly suspect, especially since a value near $1 / 3$ seems much more realistic for Poisson's ratio.

It is also of interest to consider the compressibilities. For a material with transverse isotropy, the linear compressibilities are

$$
\mathrm{k}_{3}=\mathrm{s}_{33}+\mathrm{s}_{23}+\mathrm{s}_{12}
$$

and

$$
\mathrm{k}_{1}=2 \mathrm{~s}_{12}+\mathrm{s}_{11}
$$

where the subscripts designate the axis. Also, the volume compressibility is

$$
k=2 k_{3}+k_{1}
$$

If the room-temperature values of the compliances, as given in Table 4-4, are used, then $\mathrm{k}_{1}$ is seen to be negative. Since this is unlikely, a minimum value for $\mathrm{S}_{12}$ would seem to be $\mathrm{S}_{12}=-\mathrm{S}_{11} / 2=-0.0615 \times 10^{-10} \mathrm{~N} / \mathrm{m}^{2}$. This, of course, leads to a value of $1 / 2$ for the Poisson's ratio. If the Poisson ratio $v_{21}$ is taken as $1 / 3$, then $\mathrm{s}_{12}=-0.041$ at room temperature, and $\mathrm{k}_{3}=0.179 \times 10^{-10} \mathrm{~N} / \mathrm{m}^{2}, \mathrm{k}_{1}=0.041 \times 10^{-10} \mathrm{~N} / \mathrm{m}^{2}$, and the volume compressibility $k=0.399 \times 10^{-10} \mathrm{~N} / \mathrm{m}^{2}$. These are probably reliable values for the compressibilities. This leads to a value for the bulk modulus (reciprocal compressibility) of $2.506 \times 10^{10}$ $\mathrm{N} / \mathrm{m}^{2}$. By comparison, the bulk modulus of copper is about $1.4 \times 10^{11} \mathrm{~N} / \mathrm{m}^{2} .46$

The elastic stiffnesses $\left(C_{i j}\right)$ can be directly calculated by inverting the $S_{i j}$ matrix if all the compliances are known. The explicit relationships are ${ }^{47}$ :

$$
\begin{aligned}
& C_{33}=\frac{S_{33} S_{11}-S_{12}{ }^{2}}{\left(S_{33}-S_{23}\right) \widetilde{S}}, \\
& C_{23}=\frac{S_{12}{ }^{2}-S_{23} S_{11}}{\left(S_{33}-S_{23}\right) \widetilde{S}}, \\
& C_{12}=-\frac{S_{12}}{\widetilde{S}}, \\
& C_{11}=\frac{S_{33}+S_{23}}{\widetilde{S}}, \\
& C_{55}=\frac{1}{S_{55}},
\end{aligned}
$$


and

$$
\mathrm{C}_{44}=\frac{1}{\mathrm{~S}_{44}}
$$

where $\widetilde{S}=\mathrm{S}_{11}\left(\mathrm{~S}_{33}+\mathrm{S}_{23}\right)-2 \mathrm{~S}_{12}{ }^{2}$. Except for $\mathrm{C}_{55}$ and $\mathrm{C}_{44}$, these relationships depend strongly on $\mathrm{S}_{12}$. In calculating the elastic stiffnesses, a value of $1 / 3$ for $v_{12}$ was used, and this Poisson's ratio was taken as constant with temperature. 40 It is believed that the elastic stiffnesses obtained this way are more accurate than those obtained using the resonance value of $V_{12}$. The elastic stiffnesses are given in Table 4-5.

As expected, the elastic properties of this composite are highly anisotropic. The anisotropy is best described by considering the percent elastic anisotropy as discussed by Chung and Buessem. ${ }^{48}$ For transversely isotropic symetry, this scheme involves using the conventional anisotropy ratios (the linear compressibility ratio $A_{c}=k_{1} / k_{3}$, and the shear anisotropy $A_{S}=C_{55} / C_{44}$ ) to calculate anisotropy parameters $A_{C}$ and $A_{S}$ * with the following properties: $A^{*}$ is zero for materials that are elastically isotropic, i.e., $A=I ; A^{*}$ is always positive and a single-valued measure of the elastic anisotropy of a material regardless of whether $A>1$ or $A<1$; and $A^{*}$ gives the relative magnitude of the elastic anisotropy present in the material. For this composite $A_{c} \simeq 0.23, A_{c}^{*} \simeq 8.4 \%$, $A_{S} \simeq 0.61$, and $A_{S}^{*} \simeq 12.3 \%$. For comparison, $A_{c}{ }^{*} \simeq 9.9 \%$ and $A_{S}{ }^{*} \simeq 13.7 \%$ for 2 inc, ${ }^{48}$ which is one of the most anisotropic hexagonal metals.

The static value of Young's modulus of the fiberglass-epoxy is also available. 40 This Young's modulus was measured on a specimen oriented like the one used for the resonance measurements. The resonance and static values differ by $4-10 \%$. There was no evidence of a maximum in the static data, but it is believed that the maximum is real.

\subsection{Conclusions}

The resonant piezoelectric oscillator method used in this work is generally suitable for measuring the elastic properties of composite materials. This method is accurate, fast, and requires only small specimens. It is suitable for measurements at all temperatures from $0 \mathrm{~K}$ to greater than $1000^{\circ} \mathrm{C} .^{29,30}$ The general techniques and theory of this method are well understood. This method does not appear to have the limitations of static and ultrasonic techniques.

The elastic compliance data presented in this work agree well with existing data taken by conventional static methods. These data can be used to calculate other elastic properties, such as the elastic stiffness coefficients and compressibilities. 
The superconducting coil composite is highly anisotropic, with elastic anisotropies similar to those of zinc. This high anisotropy, characteristic of many composite materials, necessitates careful measurements of elastic properties of these materials.

The measured elastic moduli of the composite are not as well represented by the Varshni function as they are for most metals and alloys; i.e., the elastic moduli of the superconducting coil composite are less regularly behaved with respect to temperature.

\section{REFERENCES}

1. P. R. Goggin, J. Mater. Sc1. $\underline{8}, 233$ (1973).

2. T. J. Dudek, J. Compos. Mater. 4, 232 (1970).

3. T. Hirai and D. E. Kline, J. Compos. Mater. , 160 (1973).

4. A. B. Schultz and Stephen W. Tsai, J. Compos. Mater. $\underline{2}, 368$ (1968).

5. R. C. Rossi, J. R. Cost, and K. R. Janowski, J. Amer. Ceramic Soc. 55,235 (1972).

6. G. C. Wright, J. Sound Vibr. 21, 205 (1972).

7. R. E. Allred and W. R. Hoover, J. Compos. Mater. $\underline{8}, 15$ (1974).

8. L. J. Cohen and O. Ishai, J. Compos. Mater. 1, 1030 (1967).

9. N. L. Hancox, J. Mater. Sci. 7, 1030 (1972).

10. T. R. Tauchert, J. Compos. Mater. $\underline{5}, 456$ (1971).

11. A. Smith, J. Phys. E. Sci. Instrum. 5, 274 (1972).

12. A. Smith, W. N. Reynolds, and N. L. Hancox, J. Compos. Mater. 7,138 (1973).

13. A. Smith, S. J. Wilkinson, and W. N. Reyrolds, J. Mater. Sc1. 9, 547 (1974).

14. J. V. Grabel and J. R. Cost, Meta11. Trans. 3, 1973 (1972).

15. A. E. Lord and D. Robert Hay, J. Compos. Mater. $\underline{6}, 278$ (1972).

16. W. Sachse, J. Compos. Mater. $\underline{8}, 378$ (1974).

17. J, O. Achenbach and G. Hermann, ALAA J. $\underline{6}, 1832$ (1968).

18. J. E. Zimmer and J. R. Cost, J. Acoust. Soc. Amer. 47, 795 (1970).

19. W. N. Reynolds and S. J. Wilkinson, U1trasonics 12, 109 (1974).

20. S. J. Wilkinson and W. N. Reynolds, J. Phys. D: App1. Phys. 7, 50 (1974).

21. T. R. Tauchert and A. N. Guzelsu, J. App1. Mech., Trans. ASME 39, 98 (1972).

22. M. F. Markham, Composites $\underline{1}, 345$ (1970).

23. G. D. Dean and P. Turner, Composites, July 174 (1973).

24. R. S. Smith, J. App1. Phys. 43, 2555 (1972).

25. A. R. Zecca and D. R. Hay, J. Compos. Mater. 4, 556 (1970). 
26. S. L. Quimby, Phys. Rev. 25, 558 (1925).

27. L. Balamuth, Phys. Rev. 45, 715 (1934).

28. F. C. Rose, Phys. Rev. $\underline{49}, 50$ (1936).

29. L. Hunter and S. Siege1, Phys. Rev. 61, 84 (1942).

30. J. W. Marx and J. M. Silvertsen, J. Appl. Phys. 24, 81 (1953).

31. M. E. Fine, Rev. Sci. Instrum. 25, 1188 (1954).

32. S. Siegel and S. L. Quimby, Phys. Rev. 49, 663 (1936).

33. J. Zacarias, Phys. Rev. 44, 116 (1933).

34. J. Marx, Rev. Sci. Instrum. 22, 503 (1951).

35. M. E. Fine, ASTM Special Tech. Publ. No. 129, 48 (1952).

36. W. H. Robinson, S. H. Carpenter and J. L. Tallon, J. Appl. Phys. 45, 1975 (1974).

37. A. J. Matheson, J. Phys, E. (Sci. Instru.) 4, 796 (1971).

38. A. P. Gerk, Rev. Sc1. Instrum. 43, 1786 (1972).

39. D. L. Johnson, University of Illinois, private communication.

40. C. W. Fowlkes, P. E. Angerhofer, R. N. Newton, and A. F. Clark, NBSIR 73-349 (1973).

41. A. F. Clark, National Bureau of Standards, private communication.

42. W. N. Robinson and A. Edgar, IEEE Trans. Sonics and U1trasonics 21, 98 (1974).

43. W. Voigt, Lehrbuch der Kristallphysik, (Teubner, Berlin, 1928), p. 746-7.

44. Y. P. Varshni, Phys. Rev. B2, 3952 (1970).

45. E. R. Naimon, W. F. Weston, and H. M. Ledbetter, Cryogenics 14, 721 (1973).

46. H. M. Ledbetter and E. R. Naimon, J. Phys. Chem. Ref. Data $\underline{3}$, (1974).

47. R. F. S. Hearmon, Rev. Mod. Phys. 18, 409 (1946).

48. D. H. Chung and W. R. Buessem, Anisotropy in Single-Crystal Compounds (Edited by F. W. Vahldiek and S. A. Mersol, Plenum, N.Y., Vol. 2, P. 217, 1968). 


\subsection{Introduction}

The NBS Cryogenics Division has been assisting the sponsor in several ways: (a) evaluation of the elastic constants of various coil and cryostat materials, for use in stress calculations, (b) evaluation of maximum allowable stresses in various coil materials, stresses which could cause either mechanical failure, or degradation of the nominal superconducting properties of the wire, and (c) representative calculation of operating stresses for a particular test coil of the sponsor using data from (a) and constraints from (b). This part of the report presents the calculations and results obtained under this last item. Stresses in superconducting magnet windings originate from four separable causes: internal stresses due to the fabrication process, (2) thermal stresses due to differential contraction between various parts as the magnet is cooled to helium temperature, (3) stresses due to the magnetic field produced by the coil, and (4) time-dependent stresses caused by localized temperature inhomogeneities or eddy-currents from changing magnetic 'fields. Reliable coil design involves, among other considerations, prediction of these stresses as a function of various material, geometrical, and operational factors, and consequent design selection such that allowable stresses are not exceeded.

The geometry of interest to the sponsor is a simple cylindrical coil wound with superconducting wire, with glass cloth separating wire layers, which is then epoxy-impregnated to form a monolithic structure. Stress analysis of such a structure may be separated into sequential steps: (1) macroscopic analysis, which assumes a uniform material characterized by anisotropic elastic and thermal moduli, and (2) microscopic analysis, in which the detailed stress distribution from one wire through the epoxy to the next wire is calculated from knowledge of the macroscopic average stress found in (1). Here we consider the first step, the macroscopic analysis.

\subsection{Consultation}

Early in this study on magnet stresses, telephone discussions on the subject were held with a number of superconducting magnet experts in government laboratories throughout the country. The purpose was to find what stress calculations, if any, they had been doing on stresses in anisotropic magnet structures. Several laboratories have the capability of 
doing this kind of calculation using sophisticated finite-element computer programs, with running times reported for some magnet studies of up to an hour on a CDC 6600 computer. Such elaborate calculations may be necessary in the final stages of a very large system design, but are hardly appropriate for exploring the general effects of properties variations and optimization of fabrication techniques.

A group at Rutherford Laboratory in England has reported studies of composite magnet materials ${ }^{1 *} \quad$ but they have not developed the macroscopic stress analysis as is done in this report.

A group at the University of hisconsin has been studying the problems of superconducting magnetic energy storage. As part of this effort, Professor Warren Young has been developing a less general but very practical progran for stress calculations in superconducting coils. After discussing the problem with Professor Young in some detail, he felt that one of their two dimensional programs could be modified to accept a specified maznet winding tension at the input. An intermediate output would be prediction of stresses up to but not including the magnetic field stresses; these would then serve as input to a much longer 3-dimensional finite element program for evaluation of axial as well as radial and circumferential stresses due to the magnetic field. This latter calculation is certainly a necessary part of the program, in that there is no hope of obtaining an analytical solution in place of the long finite-element 3-D calculation. Accordingly a small contract was given to Professor Young to perform these calculations. As of writing this report, Professor Young has incorporated the winding stresses into his 2-dimensional calculations and reported results which are compared with our analytical calculations in section 5.8 , but he has not completed the 3-dimensional magnetic field stress calculation.

Also as a result of our telephone discussions, it was decided to proceed with setting up a general analytical framework for study and evaluation of 2-dimensional stresses in anisotropic magnet coils. While the intermediate output from Professor Young's calculations will duplicate this analysis in part, the analytical study is expected to fall in the more general description of coil-winding and thermal stresses as they relate to coil fabrication techniques and material properties. The majority of this report describes this analyticai work.

\subsection{Transversely Isotropic Material Parameters in a Cylindrical Winding}

The basic material-symmetry approximation used in these calculations is that the elastic moduli and thermal expansivities are isotropic in the plane perpendicular to the wire axis, $\dot{x}$ The references in each chapter are treated separately. 
but different than the moduli parallel to the wire. This is sometimes referred to as orthotropic symmetry. It is a reasonable approximation for tightly-wound, filled, magnet coils. For our case of a cylindrically symmetric, transversely isotropic coil, strain as a function of stress is given by $2,3,4$ (nomenclature is in section 5.11).

$$
\begin{aligned}
& \varepsilon_{\theta}=\frac{\sigma_{\theta}}{E_{\theta}}-\left(\frac{v}{E}\right)\left(\sigma_{r}+\sigma_{z}\right) \\
& \varepsilon_{r}=\frac{\sigma_{r}}{E_{r}}-\left(\frac{v}{E}\right) \sigma_{\theta}-\frac{v_{t}}{E_{t}} \sigma_{z} \\
& \varepsilon_{z}=\frac{\sigma_{z}}{E_{z}}-\left(\frac{v}{E}\right) \sigma_{\theta}-\frac{\nu_{t}}{E_{t}} \sigma_{r}
\end{aligned}
$$

where

and

$$
\begin{gathered}
E_{t}=E_{r}=E_{z} \\
v_{t}=v_{r z}=v_{z r} \\
\left(\frac{v}{E}\right)=\frac{v_{\theta r}}{E_{\theta}}=\frac{v_{r \theta}}{E_{r}} .
\end{gathered}
$$

This last identity is a consequence of the fact that the strain energy is a scalar quantity. In this work we use only plane-stress $\left(\sigma_{z}=0\right)$ or plane-strain $\left(\varepsilon_{z}=0\right)$ approximations, so that the three additional equations relating torsional strain to shear stress are not needed or presented. Based on these expressions, simple algebra shows that both plane stress and plane strain relationships can be formulated as

and

$$
\varepsilon_{\theta}=\frac{\sigma_{\theta}}{\mathrm{L}}-V \sigma_{r}
$$

$$
\varepsilon_{r}=\frac{\sigma_{r}}{T}-V \sigma_{\theta}
$$

where

$$
\begin{aligned}
& L=E_{\theta}, \\
& L=\frac{E_{\theta}}{\left(1-E_{\theta} E_{t}\left(\frac{\nu}{E}\right)^{2}\right)}, \\
& T=E_{t}, \\
& T=\frac{E_{t}}{1-\nu_{t}^{2}}
\end{aligned}
$$$$
\text { for plane stress }
$$$$
\text { for plane stress }
$$$$
\text { for plane strain }
$$ 


$$
\begin{array}{ll}
V=\left(\frac{V}{E}\right), & \text { for plane stress } \\
V=\left(\frac{v}{E}\right)\left(1+v_{t}\right), & \text { for plane strain. }
\end{array}
$$

It is convenient also to express stress as a function of strain, by inverting equations $(5-4)$ and $(5-5)$,

$$
\sigma_{r}=\left(\frac{\varepsilon}{I}+V \varepsilon_{\theta}\right) \frac{1}{\Delta}
$$

and

$$
\sigma_{\theta}=\left(\frac{\varepsilon_{\theta}}{T}+V \varepsilon_{r}\right) \frac{1}{\Delta}
$$

where

$$
\Delta=\frac{1}{L T}-V^{2}
$$

Force balance in a cylindrical element of radius $r$ under either plane-stress or planestrain requires that ${ }^{1}$

$$
r \frac{\partial \sigma_{r}}{\partial r}+\sigma_{r}-\sigma_{\theta}=0
$$

\subsection{Solution for Stress Distributions in the Absence of Internal Stress}

The term "internal stress" as used here and elsewhere within this report refers to those stresses which exist within the body in the absence of all external forces applied to it. Within the elastic limit any such internal stresses are simply additive to the stresses which are produced by external forces. It is convenient here to write down the formal solution for plane-strain or plane-stress distributions in our cylindrically symetric, transversely isotropic body in the absence of such internal strain.

Following standard texts, the relationships between strain, $\varepsilon$, and an infinitesimal radial displacement, u, are:

$$
\varepsilon_{\theta}=\frac{u}{r}
$$

and

$$
\varepsilon_{r}=\frac{\partial u}{\partial r} \text {. }
$$

These expressions, rather than any of the equations (5-1) - (5-13), must be modified when internal strains are present.

Substitution of equations (5-14) and(5-15) into equations (5-10) and (5-11) yields expressions for the stresses in terms of the displacement $u$. When these latter are substituted 
into the equation of force balance, eq. (5-13), we find, after multiplying through by L:

$$
r u^{\prime \prime}+u^{\prime}-k^{2} \frac{u}{r}=0
$$

where the primes denote differentiation with respect to $r$, and the parameter

$$
k=\left(\frac{L}{T}\right)^{1 / 2}
$$

is a measure of the mechanical anisotropy of the structure. Realistic values of $k$ for epoxy-impregnated magnet windings may be in the range 1 to 3 . The solution to this differential equation is

$$
\mathrm{u}=\mathrm{ar} \mathrm{r}^{\mathrm{k}}+\mathrm{br}^{-\mathrm{k}}
$$

where $a$ and $b$ are coefficients determined by boundary conditions. Simple substitution leads to the stresses

$$
\begin{aligned}
& \sigma_{r}=\frac{a}{\Delta}\left(\frac{k}{L}+V\right) r^{k-1}-\frac{b}{\Delta}\left(\frac{k}{L}-V\right) r^{-k-1} \\
& \sigma_{\theta}=\frac{k a}{\Delta}\left(\frac{k}{L}+V\right) r^{k-1}+\frac{k b}{\Delta}\left(\frac{k}{L}-V\right) r^{-k-1} .
\end{aligned}
$$

For $k=1$, these equations reduce to the form

$$
\sigma=\text { const }_{1}+\text { const }_{2} \mathrm{r}^{-2} \text {, }
$$

which is found in standard texts for stresses in isotropic cylinders.

\subsection{Internal Stress Due to a Uniform Temperature Change}

The thermal expansion coefficients of the composite magnet winding will in general form a transversely isotropic set, just as do the elastic moduli. For a uniform temperature change $\Delta \mathrm{T}$, assume that the fractional length changes of thin samples are given by

$$
(\Delta l / \ell)_{\theta}=\alpha_{\theta} \Delta \mathrm{T}
$$

in the longitudinal (theta) direction

and

$$
(\Delta \ell / \ell)_{\mathrm{r}}=\alpha_{\mathrm{r}} \Delta \mathrm{T} \quad \text { in the transverse (radial) direction. }
$$

Because our experimental work shows that filler materials, such as epoxies, generally have higher expansion coefficients than do metals, we generally expect that $\alpha_{r}>\alpha_{\theta}$.

For our cylindrical winding, under either plane-strain or plane stress conditions, the relationships between strain, expansion coefficient, and an infinitesimal radial expansion $u(r)$ are 


$$
\begin{aligned}
& \varepsilon_{\theta}=\frac{u}{r}-\alpha_{\theta} \Delta \mathrm{T} \\
& \varepsilon_{r}=\frac{\partial u}{\partial r}-\alpha_{r} \Delta T .
\end{aligned}
$$

For an isotropic structure, with $\alpha_{\theta}=\alpha_{r}$, a uniform temperature change produces a radial expansion given by $\mathrm{u}=\mathrm{r} \alpha \Delta \mathrm{T}$, but internal stresses and strains remain at zero.

As in section 5.4, substitution of equations (5-21) and (5-22) into (5-10) and (5-11), and from there into (5-13), yields the differential equation

$$
r u^{\prime \prime}+u^{\prime}-k^{2} \frac{u}{r}=\left[\alpha_{r}-k^{2} \alpha_{\theta}+L V\left(\alpha_{\theta}-\alpha_{r}\right)\right] \Delta T
$$

Neglecting the temperature variation of the elastic moduli, the solution to this differential equation is

$$
u=a r^{k}+b r^{-k}+\frac{A \Delta T}{1-k^{2}} r
$$

where $A$ is the expression in brackets on the right-hand side of equation (5-23). Further substitution into (5-21) and 5-22), and then into (5-10) and (5-11) yields

$$
\sigma_{r}=c r^{k-1}-d r^{-k-1}+\frac{L\left(\alpha_{r}-\alpha_{\theta}\right) \Delta T}{1-k^{2}}
$$

and

$$
\sigma_{\theta}=k c r^{k-1}+k d r^{-k-1}+\frac{L\left(\alpha_{r}-\alpha_{\theta}\right) \Delta \mathrm{T}}{1-k^{2}}
$$

and $c$ and $d$ are a different pair of arbitrary constants. The thermal stresses are those which are present in the absence of external forces, i.e., at the inner $(r=1)$ and outer $(r=g)$ boundaries, when

$$
\sigma_{r}(1)=0=\sigma_{r}(g)
$$

Evaluation of $c$ and $d$ for this condition is straightforward, and both are found to be proportional to the quantity

$$
\mathrm{L}\left(\alpha_{\mathrm{r}}-\alpha_{\theta}\right) \Delta \mathrm{T} \equiv \mathrm{L} \Delta \alpha \Delta \mathrm{T}
$$

Thus it is convenient to normalize the internal stresses $\sigma_{\varepsilon}$ and $\sigma_{\theta}$ by dividing through by this quantity. Within the elastic limit, the thermal stresses are simply added to any externally-produced stresses to obtain the total stresses on the winding under operating conditions. 
At first sight the equations appear to blow up as $\mathrm{K} \rightarrow 1$, i.e., for an isotropic material -- a most unphysical result. However, expansion of the equations in terms of the small quantity $(k-1)$ shows that in this limit the equations reduce to the form

$$
\frac{\sigma}{\mathrm{L} \Delta \alpha \Delta \mathrm{T}}=\text { const }_{1} \cdot \operatorname{lnr}+\text { const }_{2} \cdot \mathrm{r}^{-2}+\text { const }_{3}
$$

where the constants are independent of $\mathrm{k}$. This is the form given by standard texts, e.g., Timoshenko and Goodier ${ }^{5}$, for internal stresses in isotropic cylindrical geometries.

Numerical evaluation of these equations for a particular coil geometry, set of material properties, and cooldown from 338 to $4 \mathrm{~K}$ is shown in figure 5-1. Three features of these curves should be noted: (a) Maximum circumferential compressive stress occurs at the outside radius, (b) Maximum circumferential tensile stress occurs at the inside radius, and (c) Maximum radial tensile stress occurs within the body of the coil, but is generally smaller in magnitude than the maximum circumferential stresses.

Generalized representation of these three maximum stresses as a function of coil shape $\left(r_{0} / r_{i}\right)$ and mechanical modulus anisotropy $(k)$ is shown in figures 5-2, 5-3, and 5-4, al1 normalized to the thermal expansion anisotropy (L $\Delta \alpha \Delta \mathrm{T}$ ). For practical epoxy-impregnated windings, cooled from a curing temperature above room temperature down to $4 \mathrm{~K}$, (L $\Delta \alpha \Delta \mathrm{T}$ ) may wel1 be as large as $-35 \times 10^{3} \mathrm{~N} / \mathrm{cm}^{2}$, or $-50 \times 10^{3}$ psi. One can see from the figures that the maximum internal stresses due to cooldown of practical superconducting magnet coils may typically be in the range 0.05 to 0.5 of this number, and thus may be quite significant. On a macroscopic scale, these thermal stresses in principle could be reduced to zero by adding a filler to the epoxy such that its expansivity matches that of the superconducting wire, or $\alpha_{r}=\alpha_{\theta}$. There may even be an advantage in carrying this further, to obtain $\alpha_{r}<\alpha_{\theta}$ (though in practice this would be very difficult to achieve). Under this condition, dool-down of the coil would cause compressive circumferential stress at the inner radius, and tensile stress at the outer radius. If such a stress distribution were added to the stresses subsequently generated by the magnetic field, it would tend to produce a more uniform circumferential stress distribution throughout the coil, rather than having it peaked near the inner radius where the magnetic field is highest.

\subsection{Stresses due to Winding Tension}

We consider the layer-by-layer winding of a simple solenoid in which relatively soft insulating material, such as fiberglass, is wrapped between each layer of superconducting 


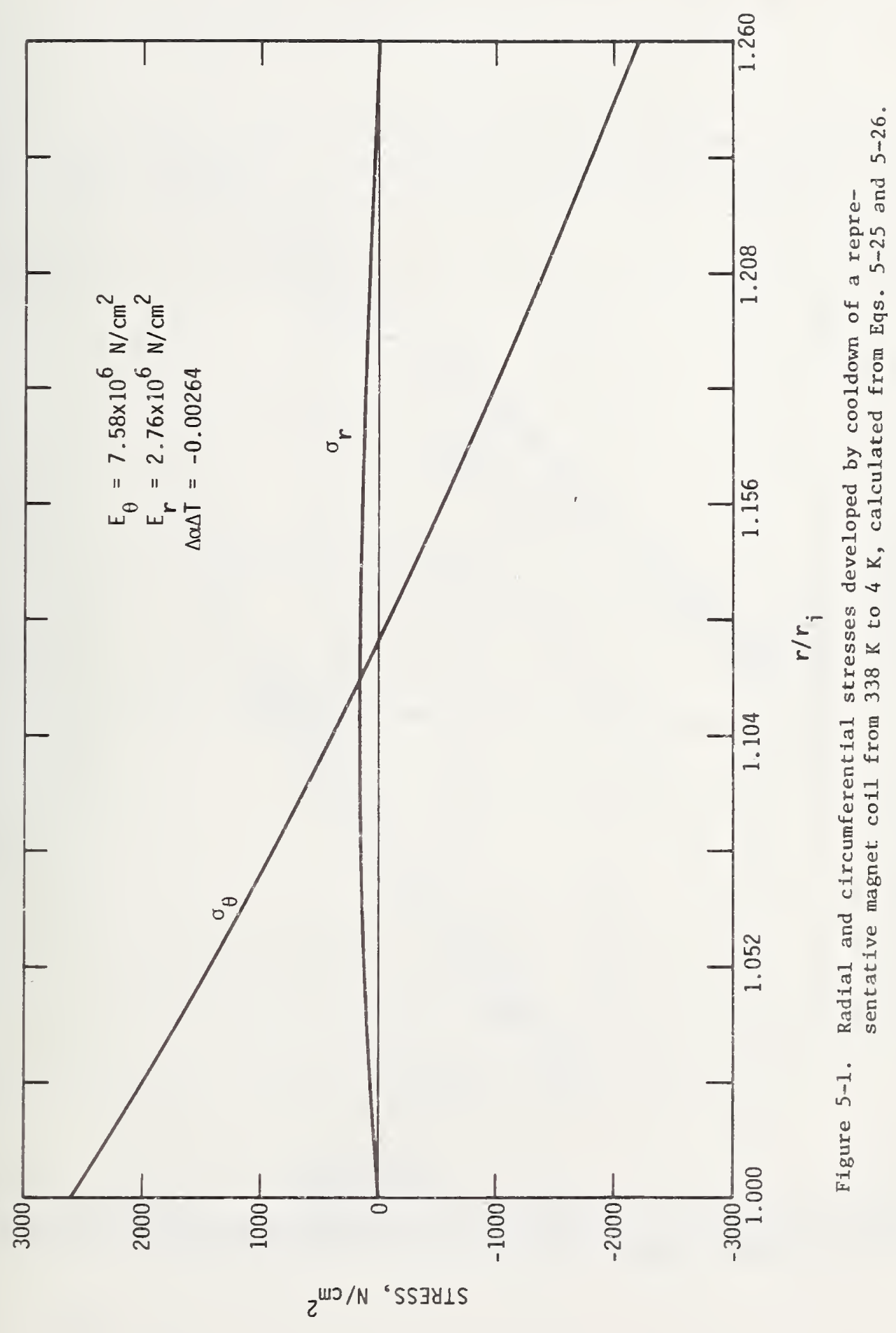




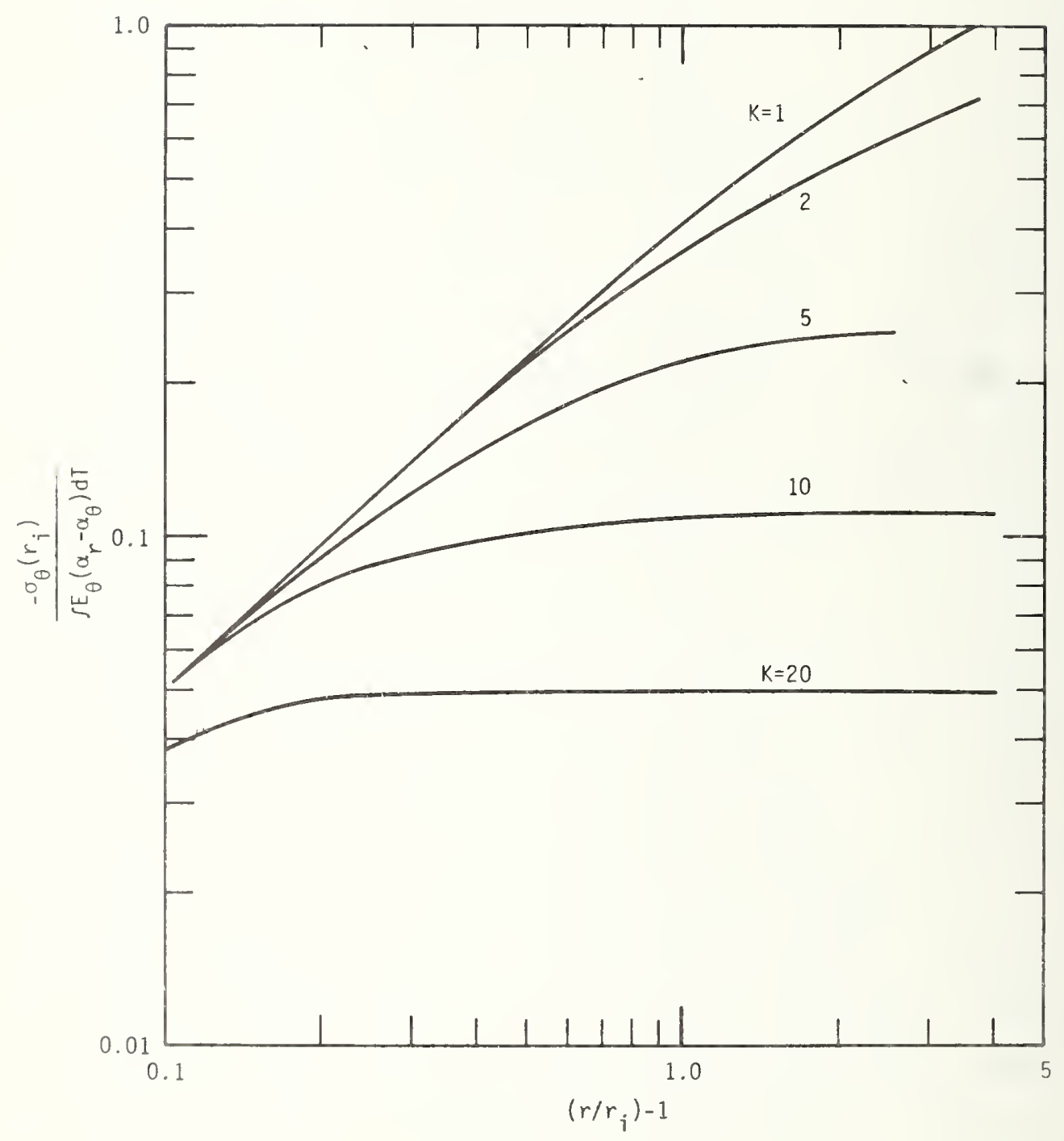

Figure 5-2. Circumferential stress developed at the inner radius due to a uniform temperature change, normalized by the parameter $L \Delta \alpha \Delta T$ (Eq. 5-26), as a function of coil thickness, for several values of the mechanical anisotropy parameter $\mathrm{K}$ (Eq. 5-17). 


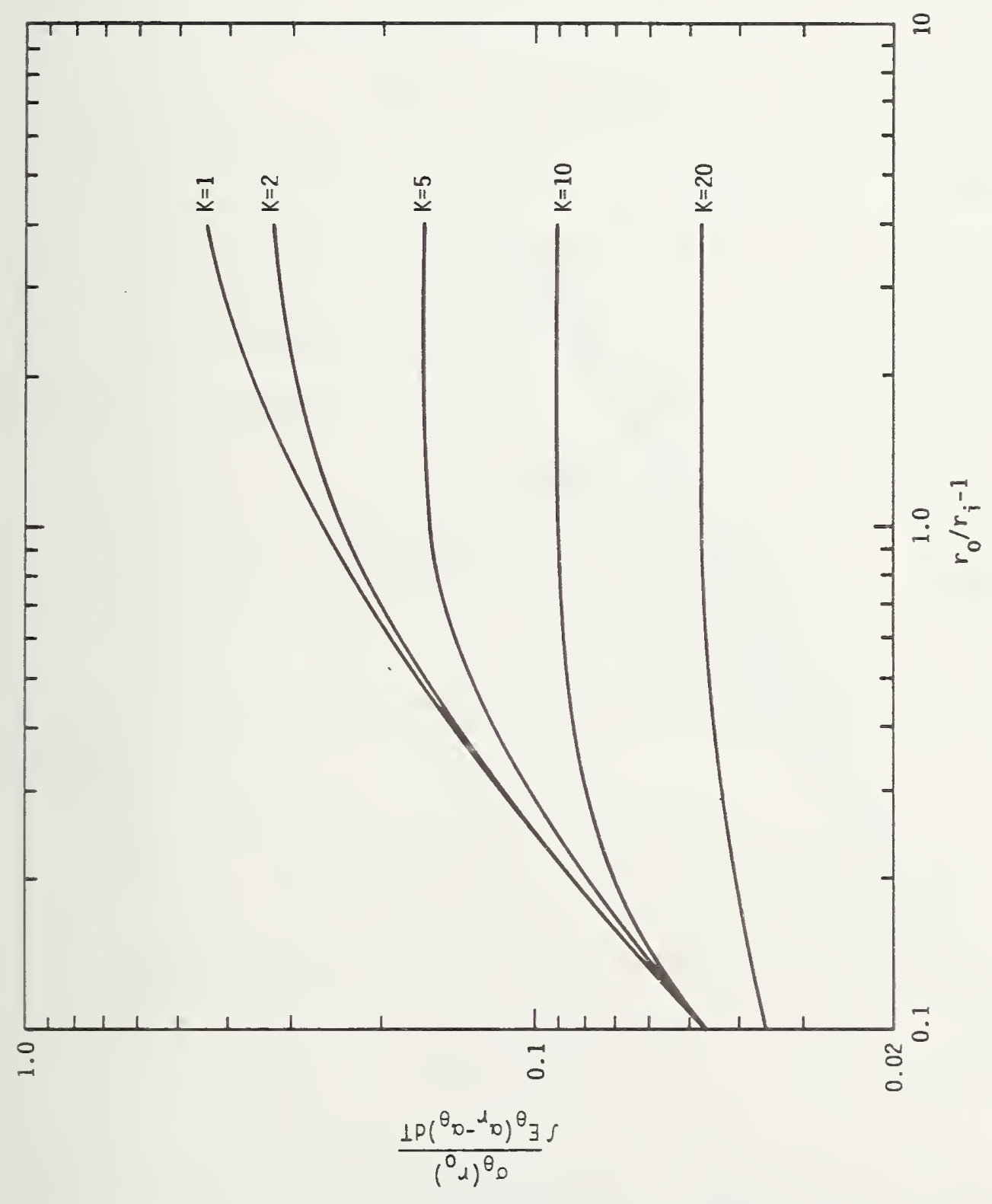

일

递出宁 $+x$ E⿱ 与。 ๙ $\therefore$ ㅇํㅇ 占范 党 $\dot{\vec{\omega}}$ 它 स

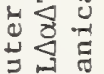
곤 造 ป气 잉 品 崩 0 D 0. 吸?

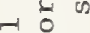
तु E) 出 i E บ $0 \frac{x}{0}$ 岁 in

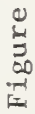




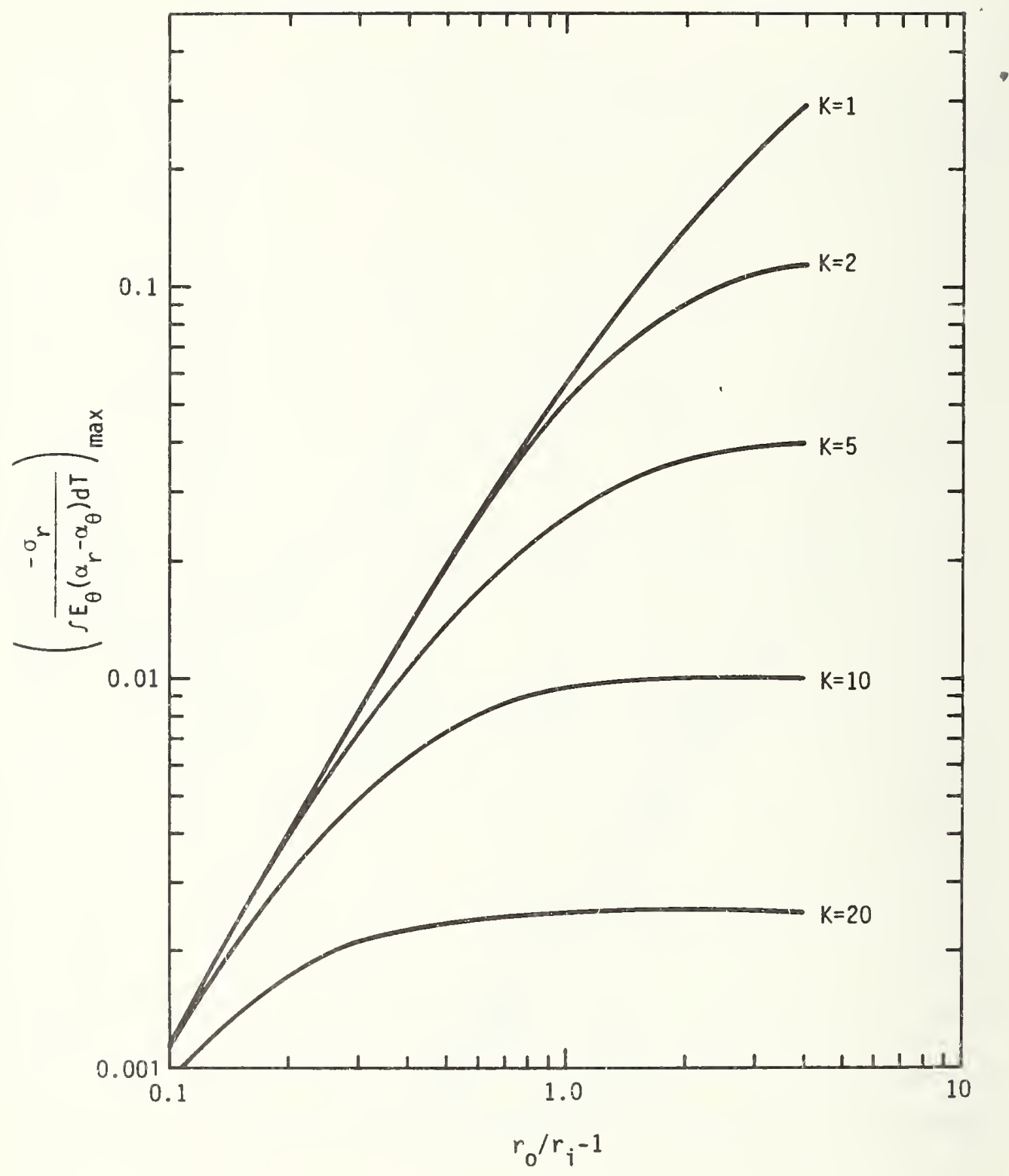

Figure 5-4. Maximum radial stress due to a uniform temperature change, normalized by the parameter $\mathrm{L} \Delta \alpha \Delta \mathrm{T}$ (Eq. 5-26), as a function of the coil thickness, for several values of the mechanical anisotropy parameter K (Eq. 5-17). 
wire. During this process the wire is maintained under tension, which we allow to vary as a function of the dimensionless radius, $r$. For tractable analysis, we make the following reasonable assumptions and approximations:

(1) After each layer is wound, the composite winding at that moment may be characterized on a macroscopic basis by a set of orthotropic material parameters (which can be estimated from the mechanical properties of the wire and the cloth). This further implies (a) that all strains are elastic, and (b) that friction between the wire and the cloth is high enough to prevent sliding of the wire along its length.

(2) As each layer is completed, there is no variation in either stress or elastic moduli in the axial direction. This allows either plane-stress or plane-strain approximations to be used. Considering that each turn falls into place under relatively low axial stress, compared to the winding tension, the plane-stress approximation is probably the better one, though numerical results are little influenced by this choice.

(3) The coil is wound on a cylindrical bobbin which is characterized by isotropic elastic moduli. Mathematically we define

$$
\begin{aligned}
& \alpha_{B}=\frac{\text { inside diameter }}{\text { outside diameter }} \text { of the bobbin } \\
& \nu_{B}=\text { Poisson's ratio of the bobbin } \\
& E_{B}=\text { Young's modulus of the bobbin. }
\end{aligned}
$$

Following standard texts, the relationship between radial deflection $\mathrm{u}_{\mathrm{B}}$ of the outside diameter and radial stress $\sigma_{r B}$ at the $0 . d$. is

$$
\frac{u_{B}}{\sigma_{r B}}=\frac{1}{E_{B}}\left(\frac{1+\alpha_{B}^{2}}{1-\alpha_{B}^{2}}-v_{B}\right) \frac{1}{B} \cdots \cdot
$$

Thus, $B$ is an effective modulus of the bobbin.

As each layer is wound onto the coil it adds a compressive stress to the layers underneath it, causing them to move radially inward by a small amount. The inward movement causes a proportionate reduction in the circumferential stress which was originally established by the winding tension. The radial compressive stress is infinitesimal at the outer layer, accumulating to substantial values at the innermost layer. However, radial deflection of this innermost layer will be very small if the bobbin is quite rigid, while radial deflection 
of the middle layers will be determined much more by the radial compressibility of the composite winding itself (which may be very high because the glass cloth interlayer is soft compared to metals).

Analysis of internal strains due to winding tension can follow either of two methods:

(1) We can use an integration technique, adding winding thickness $\mathrm{dr}$ at each increment until the final diameter is reached, or (2) we can deduce the form of the equations for the stress (and strain) distributions and evaluate arbitrary constants from boundary conditions. In this report we use method (2). However, this method proves to be an approximation: see note added in proof, p. 121 .

We assume that the programmed winding tension produces a strain $\varepsilon_{W}(r)$ given by a power series

$$
\varepsilon_{W}(r)=\sum_{0}^{n} s_{i} r^{i}
$$

when $s_{i}$ are constants which can be evaluated by fitting a polynomial to the programmed winding tension. The corresponding circumferential strain within the body of the coil is

$$
\varepsilon_{\theta}=\frac{u}{r}+\sum_{0}^{n} s_{i} r^{i}
$$

where, $u$ is the radial deflection of any given layer due to the compressive load of subsequently-wound overlayers and by definition, $u=0$ at $r=g$, the outer surface of the coil. The corresponding expression for radial strain is

$$
\varepsilon_{r}=\frac{\partial u}{\partial r}+\sum_{0}^{n} \beta_{i} r^{1}
$$

where $\beta_{i}$ are unknown constants which in fact turn out to be dependent on boundary conditions. Following the same mathematical steps outlined in section 5.3, utilizing equations (5-4) (5-12), we obtain the differential equation

$$
u^{\prime \prime} r+u^{\prime}-k^{2} u=-\sum_{0}^{n}\left[(1+i-L V) \beta_{i}+\left((1+i) L V-k^{2}\right) s_{1}\right] r^{1}
$$

The solution to this is found to be

$$
u=a r^{K}+b r^{-K}-\sum_{0}^{n}(i+i-L V) c_{i} r^{1+1}-\sum_{0}^{n} s_{i} r^{1+i}
$$

where the unknown $c_{i}$ 's are related to the unknown $\beta_{i}^{\prime}$ 's in eq. (5-29) by 


$$
c_{i}=\frac{(1+i) s_{i}-\beta_{i}}{k^{2}-(1+i)^{2}} \text {. }
$$

The corresponding stresses are found to be

$$
\sigma_{r}=\frac{a}{\Delta}\left(\frac{k}{L}+V\right) r^{k-1}-\frac{b}{\Delta}\left(\frac{k}{L}-V\right) r^{-k-1}-L \underset{0}{\sum} c_{i} r^{i}
$$

and

$$
\sigma_{\theta}=\frac{k a}{\Delta}\left(\frac{k}{L}+V\right) r^{k-1}+\frac{k b}{\Delta}\left(\frac{k}{L}-V\right) r^{-k-1}-L \sum_{0}^{n}(1+i) c_{i} r^{i} .
$$

The boundary conditions which must be met are:

(1) There is no radial stress at the outer surface, or

$$
\sigma_{r}(g)=0
$$

(2) The deflection, $u$, is so defined by equation (5-23) that it must be zero at the outer surface, or

$$
\mathrm{u}(\mathrm{g})=0
$$

(3) The ratio $u / \sigma_{r}$ at the inner surface must match that of the bobbin, given by equation (5-27), or

$$
\frac{u(1)}{\sigma_{r}(1)}=\frac{1}{B}
$$

Now, however, we have a procedural problem, since we have 3 boundary conditions and $n+3$ unknown constants where $(n+1)$ is the number of terms in the polynomial expression of the programed winding tension. However, the final stress distribution is uniquely determined by the given conditions, and the apparent excess of arbitrary constants in method (2) demands additional manipulation but not additional boundary conditions.

The way to continue with method (2) is to recognize that we are working within the domain of linear stress-strain theory, and that stresses from separate external forces are additive. This suggests that we rewrite equations $(5-31,33$, and 34 ) in the form 


$$
\begin{aligned}
& u=\sum_{0}^{n} u_{i}=\sum_{0}^{n}\left[a_{i} r^{k}+b_{i} r^{-k}-\left((1+i-L V) c_{i}-s_{i}\right) r^{i}\right] \\
& \sigma_{\mathrm{r}}=\sum_{0}^{\mathrm{n}} \sigma_{\mathrm{ri}}=\sum_{0}^{\mathrm{n}}\left[\frac{\mathrm{a}_{i}}{\Delta}\left(\frac{\mathrm{k}}{\mathrm{L}}+\mathrm{V}\right) \mathrm{r}^{\mathrm{k}-1}-\frac{\mathrm{b}_{i}}{\Delta} \quad\left(\frac{\mathrm{k}}{\mathrm{L}}-\mathrm{V}\right) \mathrm{r}^{-\mathrm{k}-1}-\mathrm{Lc}{ }_{i} \mathrm{r}^{i}\right] \\
& \sigma_{\theta}=\sum_{0}^{\mathrm{n}} \sigma_{\theta i}=\sum_{0}^{\mathrm{n}}\left[\frac{\mathrm{ka}}{\Delta}\left(\frac{\mathrm{k}}{\mathrm{L}}+\mathrm{V}\right) \mathrm{r}^{\mathrm{k}-1}+\frac{\mathrm{b}_{i}}{\Delta}\left(\frac{\mathrm{k}}{\mathrm{L}}-\mathrm{V}\right) \mathrm{r}^{-\mathrm{k}-1}-\mathrm{L}(1+i) \mathrm{c}_{i} \mathrm{r}^{\mathrm{i}}\right] .
\end{aligned}
$$

We then require that each of the $n$ components of strain $u_{i}$ and stress $\sigma_{r i}$ obey the boundary conditions (5-30-32), -- a procedure which will be justified at a later stage. When this is done for the $i^{\text {th }}$ component, we obtain equations of the form

$$
\begin{array}{r}
g^{k} a_{i}+g^{-k_{b}}+\quad(L V-1-i) c_{i}=s_{i} g^{2} \\
\left(\frac{k}{L}+V\right) \frac{g^{k-1}}{\Delta} a_{i}-\left(\frac{k}{L}-V\right) \frac{g^{-k-1}}{\Delta} b_{i}- \\
L g^{i} c_{i}=0 \\
\left(1-\frac{\left(\frac{k}{L}+V\right)}{B \Delta}\right) a_{i}+\left(1+\frac{\frac{k}{L}-V}{B \Delta}\right) b_{i}+\left(\frac{L}{B}+L V-1-i\right) c_{i}=s_{i} .
\end{array}
$$

The constants $a_{i}, b_{i}$, and $c_{i}$ are readily determined from these three equations. An immediate result, from the form of equations (5-41), is that for the general case

$$
a_{i}=b_{i}=c_{i}=0 \text { if } s_{i}=0
$$

For exampie, if the coil is wourd at constant tension,

$$
s_{0} \neq 0, s_{i}=0 \text { for } i>0
$$

we obtain equations equivalent to $(5-25)$ and $(5-26)$ in their dependence on $r$ :

$$
\begin{aligned}
& \sigma_{r}=C^{1 k-1}-D r^{-k-1+D_{o}} \\
& \sigma_{\theta}=k C r^{k-1}+k D r^{-k-1}+D_{0} .
\end{aligned}
$$

On the cther hand, if a higher order polynomial, of degree $n$, must be used to describe the programmed winding tension, then in eqs. (5-42) and (5-43)

$$
\begin{aligned}
& C=\frac{1}{\Delta}\left(\frac{k}{L}+V\right) \sum_{0}^{n-1} a_{i}, \\
& D=\frac{1}{\Delta}\left(\frac{k}{L}-V\right) \sum_{0}^{n-1} b_{i},
\end{aligned}
$$


and for each non-zero $s_{i}$ an additional term $D_{i} r^{i}=L c_{i} r^{i}$ must be added to the right hand side of equation (5-42), and $(1+i) D_{i} r^{i}$ must be added to the right hand side of equation $(5-43)$.

The justification for the unusual procedure of applying boundary conditions to each of the $i^{\text {th }}$ components $u_{i}$ and $\sigma_{i}$ in equations (5-38) to (5-40), rather than just to the sums $u=\sum u_{i}$ and $\sigma=\sum \sigma_{i}$, is that in doing so we have obtained a final solution which fits all physical conditions to the problem, namely plane-stress orthotropic material behavior (eqs $(5-5,6))$, force balance (eq (5-13)), programmed winding tension (eq (5-28)) and boundary conditions at the inner and outer radius, (eqs $(5-35,36,37)$ ). Since on a physical basis these conditions to the problem are sufficient to determine a unique stress and strain distribution within the coil, this mathematical solution must likewise be unique.

Figure 5-5 shows circumferential and radial stresses calculated from these equations for a particular coil geometry, assuming a constant strain of 0.001 due to winding tension, i.e.,

$$
\varepsilon_{\theta}=u / r+0.001
$$

Multiplying this by the longitudinal modulus, L, yields the corresponding winding stress, which is plotted in figure $5-5$ as the dotted line. This applied winding stress must equal the calculated circumferential stress at the outer radius, where there is no overwinding to cause stress relaxation. In the example of figure 5-5, circumferential stress relaxation of up to $55 \%$ has occurred within the body of the coil during the winding process. A significant test of the numerical results is to approximate the constant winding tension of the above numerical example by the expression

$$
\varepsilon_{\theta}=u / r+.002676 r-.002379 r^{2}+.0007025 r^{3}
$$

which differs in magnitude from equation (5-46) by less than $0.04 \%$ when $r$ is in the range from 1 to 1.26, appropriate to the sponsors test coil. Thus, physically it describes essentially the same programmed winding tension as was used for the example of figure 5-1, but mathematically its solution invokes equations (5-38-40), with $\mathrm{n}=3$, rather than the simpler equations (5-31-34) appropriate to a constant winding tension. We find that the calculated stresses obtained with equation (5-47) agree within $0.1 \%$ with those used in 


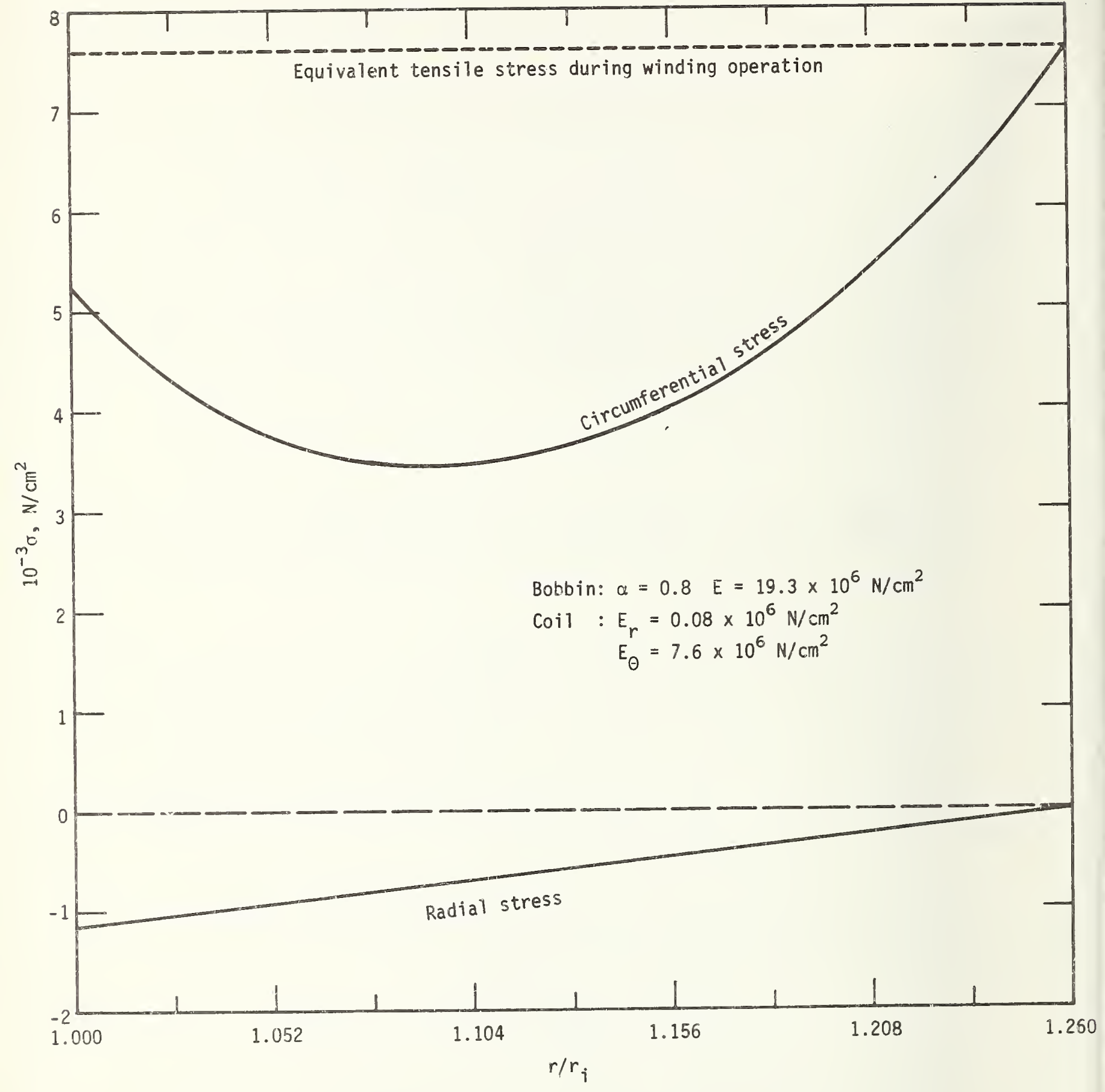

Figure 5-5. Radial and circumferential stress calculated from Eqs. 5-42, 43 for a particular co:1 wound at constant strain of 0.001 , equivalent in this example to a tensile stress of $7.6 \mathrm{kN} / \mathrm{cm}^{2}$, shown as the dashed line. Because of the very low value of $E_{r}$, strain reiaxation of up to $55 \%$ occurs within the winding. 
plotting figure 5-5. This further confirms the validity of the analytical assumptions which were made in extending equations (5-31-34) to the more general form of equations $(5-38-40)$.

Another test of the analysis is made by assigning an artifically high modulus to the bobbin, such that it becomes almost infinitely rigid. Under this condition the winding strain at $\mathrm{r}=1$ cannot change as subsequent layers are overwound, or in other terms, $u(1)=0$, regardless of the shape and magnitude of the programmed winding strain curve away from the point $r=1$. Calculated results, not shown, are in agreement with this prediction.

\subsection{Epoxy Impregnation of the Coil and Bobbin Removal}

After being wound, the coil is impregnated with epoxy to form a rigid, monolithic structure. This takes place at an elevated temperature, the curing temperature of the epoxy. The change in stress as the unimpregnated coil is warmed from room temperature to the curing temperature, and recooled to room temperature after impregnation can be calculated separately using the results of section 5.5 .

We assume that the coil stresses do not change during the curing operation. Actually the epoxy may shrink during the curing operation, resulting in some change in the stresses, but in practice the change in stress distribution is probably not large. This point needs further study, which we will address in the future.

Assume that the stresses in the coil at the beginning of the impregnation process are known. Since we are working within the framework of elastic behavior, these stresses can be uniquely divided into (a) "internal" stresses satisfying the boundary conditions $\sigma_{r}(1)=0$ and $\sigma_{r}(\gamma)=0$, and (b) "external" stresses, due to the compressive pressure P of the bobbin on the inside diameter of the coil. When the bobbin is removed, the "external" stresses disappear, leaving just the "internal" stress.

The stresses due to a compressive pressure $\mathrm{P}$ at the inside diameter are easily determined from equation (5-19) by applying the boundary conditions

$$
\sigma_{r}(1)=-P \quad, \quad \sigma_{r}(\gamma)=0
$$

to determine the two unknown coefficients in the $\sigma_{r}$ equation. The same coefficients occur in equation (5-20), so that the circumferential stress distribution $\sigma_{\theta}$ is likewise determined. The material property, $k$, which appears in these two equations is that of the composite coil 
after epoxy impregnation. When the bobbin is removed, the two stress distributions $\sigma_{r}$ and $\sigma_{\theta}$, so calculated, are simply subtracted from the total stresses which existed as the beginning of the impregnation process. The corresponsing dimensional change of the bobbin is

$$
\Delta u=r \Delta \varepsilon_{\theta}=r\left(\frac{\Delta \sigma_{\theta}}{L}-v \Delta \sigma_{r}\right)
$$

This process of epoxy impregnation and bobbin removal results in a general reduction of $\sigma_{\theta}(r)$ from that established by the winding process, such that

$$
\int_{1}^{g} \sigma^{\prime} \theta^{(r)}=0 \text {. }
$$

Since in general, for the coils we consider, $\sigma_{r}$ is much less than the $\sigma_{\theta}$ due to winding tension, the curve $\sigma_{\theta}(r)$ does not change shape greatly during this process. This change is illustrated in figure 5-6, which shows the initial $\sigma_{\mathrm{r}}$ and $\sigma_{\theta}$, taken from figure 5-5, and the final $\sigma_{\mathrm{r}}$ and $\sigma_{\theta}$ after epoxy impregnation and bobbin removal, calculated from the above equations for a particular model coil.

\subsection{Magnetic Forces}

The magnetic field generated by a cylindrical coil will be a function of both $r$ and $z$, resulting in a stress distribution which may be significantly different from either the (2-dimensional) plane stress or plane strain solutions which we have used up to this point. The only practical way to obtain complete answers to this problen is to use a 3-dimensional, finite-element analysis. However, for at least some geometries, the axial stresses will be somewhat smaller than the circumferential tensile stresses, and our simple 2-dimensional analysis will provide a rough approximation to the stress distribution at the midplane where $\sigma_{\theta}$ is largest. Furthermore, changes in the operating stresses following changes in fabrication techniques and/or material properties can perhaps be estimated to reasonable accuracy even though the true stress distribution may be more uncertain. We give here the 2-dimensional solution for magnetically-induced stresses as the midplane of our or thotropic magnet winding.

The magnetic field causes a body force $\mathrm{dF}_{\mathrm{r}}$ on every element of the coil, proportional to the vector cross-product of field and current. Considering just the axial component of magnetic field, which produces a radial force, eq (5-13) becomes 5,6 


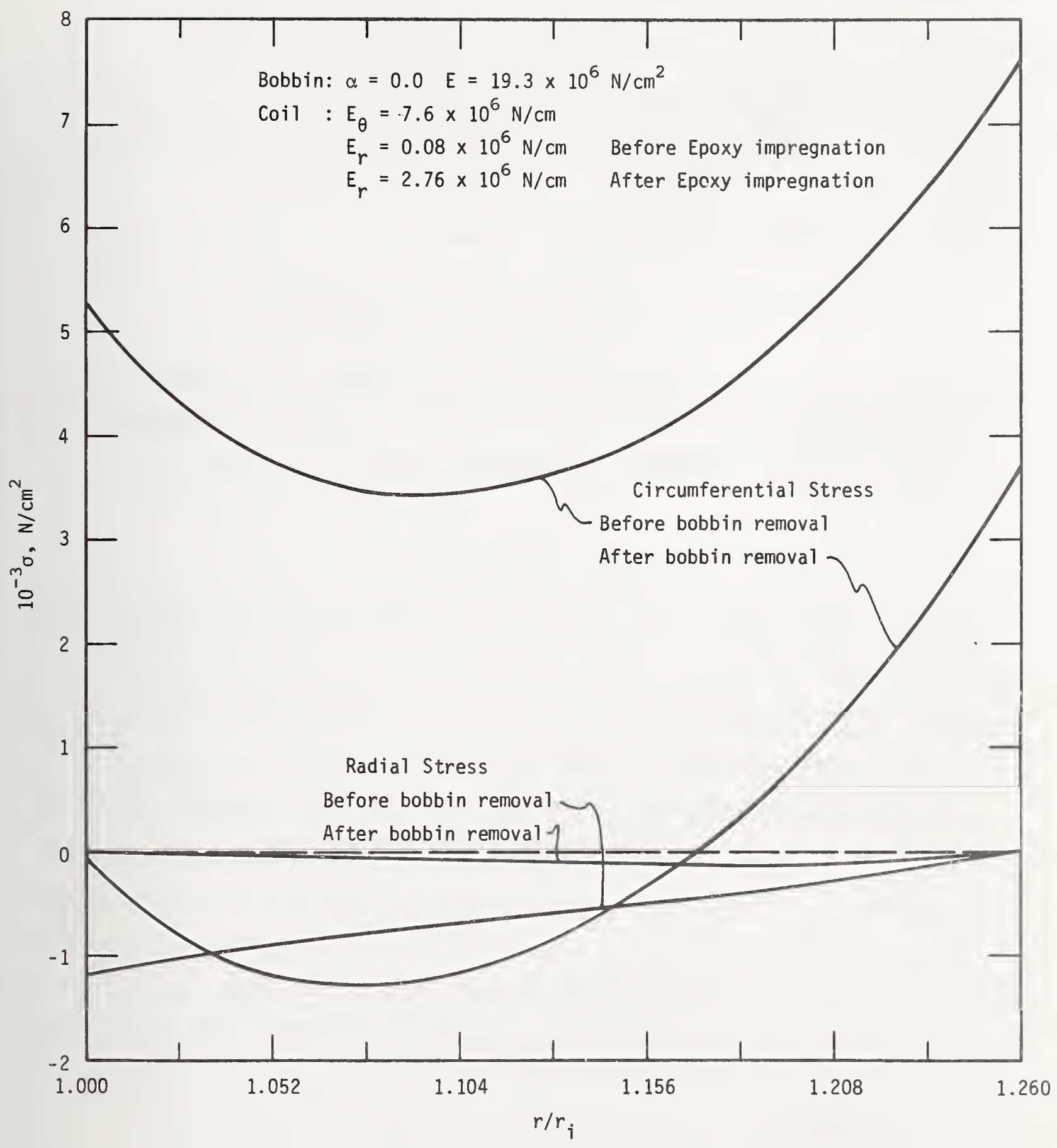

Figure 5-6. Radial and circumferential stresses for a particular coil as wound, and after epoxy impregnation and removal of the bobbin on which the coil was wound. 


$$
r \frac{\partial \sigma_{r}}{\partial r}+\sigma_{r}-\sigma_{\theta}=-r \frac{\partial F}{\partial V}
$$

For a rectangular coil cross-section carrying a uniform current density,

$$
r \frac{{ }^{d}{ }_{r}}{d r}=\left[\frac{B_{0}^{2} r}{4 \pi \phi(g-1)}\right] \frac{B_{z}}{B_{o}}=H \frac{B_{z}}{B_{o}} r
$$

where $B_{0}$ is the magnetic field at the center of the coil, and

$$
\phi=\frac{\beta}{g-1} \ln \left(\frac{g+\left(g^{2}+\beta^{2}\right)^{1 / 2}}{1+\left(1+\beta^{2}\right)^{1 / 2}}\right)
$$

is the Fabry factor. In this expression $\beta$ is the coil length divided by inside diameter. The parameter $\mathrm{H}$, defined by eq $5-49$, is proportional to the square of the magnetic field. Consistent with the accuracy of the 2-dimensional calculation itself, we may take

$$
\frac{\mathrm{B}_{\mathrm{z}}}{\mathrm{B}_{\mathrm{O}}} \approx \mathrm{h} \frac{\mathrm{g}-\mathrm{r}}{\mathrm{g}-1} \text {, }
$$

i.e., assume that $B_{z}$ falls off lineraly from the value $h B_{0}$ ( $h \approx 1$ to 2 , depending on $\beta$ and g) at the inner surface of the windings to zero at the outer surface. The parameter $h$, defined by eq 5-50, is the field at the inner radius divided by the field at the center of the coil; for the sponsors test coil, $\mathrm{h} \approx 1.53$.

Now, following the procedure of section 5.4, the components of stress due to the magnetic field can be obtained by solving the differential equation

$$
u^{\prime \prime} r+u^{\prime}-k^{2} \frac{u}{r}=-\frac{\text { LHhg }}{g-1} r+\frac{L H h}{g-1} 4^{2} .
$$

The general solution to this equation is

$$
u=a r^{k}+b r^{-k}+c r^{2}+d r^{3}
$$

where $c=\frac{\text { LHhg } \Delta}{(g-1)\left(k^{2}-4\right)}$

and $\quad d=\frac{L H h \Delta}{(g-1)\left(k^{2}-9\right)}$.

The corresponding stresses are

$$
\sigma_{\mathrm{r}}=A \mathrm{r}^{\mathrm{k}-1}-B \mathrm{C}^{-\mathrm{k}-1}+\left(\frac{2}{\mathrm{~L}}+\mathrm{V}\right) \frac{\mathrm{c}}{\Delta} \mathrm{r}+\left(\frac{3}{\mathrm{~L}}+\mathrm{V}\right) \frac{\mathrm{d}}{\Delta} \mathrm{r}^{2}
$$




$$
\sigma_{\theta}=k A r^{k-1}+k B r^{-k-1}+\left(\frac{1}{T}+2 V\right) \frac{c}{\Delta} r+\left(\frac{1}{T}+3 V\right) \frac{d}{\Delta} r^{2}
$$

where, as before

$$
a=\frac{a}{\Delta}\left(\frac{k}{L}+v\right)
$$

and

$$
B=\frac{b}{\Delta}\left(\frac{k}{L}-V\right)
$$

These two integration constants must be evaluated from the two boundary conditions,

$$
\sigma_{r}(1)=0=\sigma_{r}(g)
$$

Evaluation is straightforward. All coefficients are proportional to $\mathrm{B}_{0}^{2}$, as is anticipated. Numerical evaluation of these magnetic stresses for a particular coil is shown in figure 5-7. Further work discloses that the calculated stresses are remarkably independent of material anisotropy parameter, $k$, and the Poisson's ratio. Varying $k$ between 1 and 3 , and $\mathrm{V}$ between zero and $0.4 / \mathrm{L}$ changes the calculated circumferential stresses by only about $1 \%$, which is far smaller than the probable accuracy of the 2 -dimensional calculation itself. The radial stress exhibits greater variation but at the same time it is generally one or more orders of magnitude smaller than the circumferential stress, and hence probably inaccurate in the face of axial compressive stresses which have been neglected in the 2-dimensional calculation. These numerical results agree with those presented by Hord [15] for an isotropic coil winding in the same two-dimensional approximation.

Greater sensitivity to $k$ and $V$ would probably be found if the coil were overwound with a load-bearing wrap carrying no current. In this case there would be a finite $\sigma_{r}$ at $r=g$ which would reduce $\sigma_{\theta}$ within the coil by an amount dependent on both coil and wrap parameters. We have not performed this calculation, but plan to do so within the next reporting period. We conclude that, within the two-dimensional approximation, and for cylindrical coils unsupported by external wraps, previously existing magnetic stress analyses based upon isotropic magnet winding structure are satisfactorily accurate for this stage of our calculations. Improvement in the accuracy of the calculated stresses due to the magnetic field requires primarily a three-dimensional calculation (using finite element techniques). Results from these calculations (Professor Young's work) will be reported later, when they become available. 


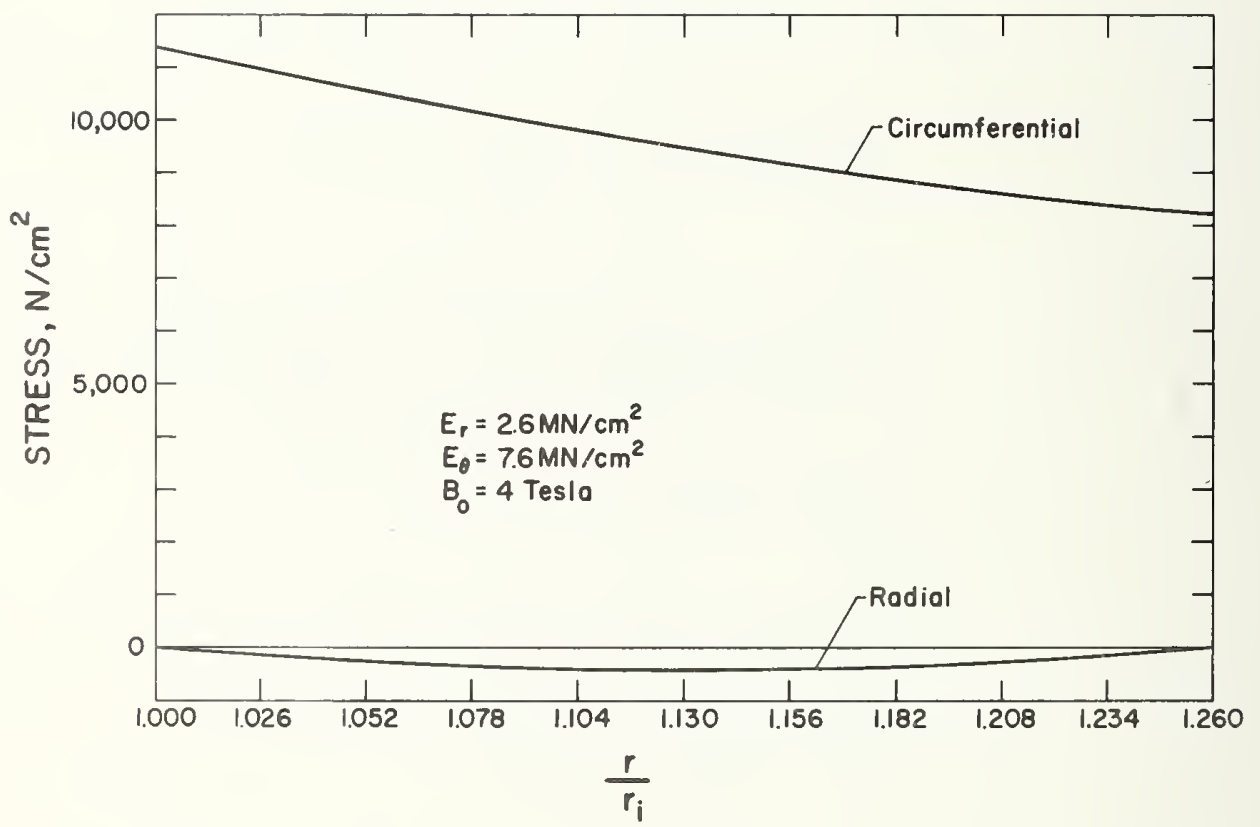

Figure 5-7. Radial and circumferential stresses for a particular coil when it generates a field of 3 Tesla at the coll center. As discussed in the text, these results are quite insensitive to reasonable variations in coil material parameters. 


\subsection{Summary of Numerical Results}

Within the framework of elastic stress-strain behavior used in this study, the cumulative stresses and strains under operating conditions will be just the sum of the stresses and strains developed at each stage of the fabrication and operation procedure. Thus, it is meaningful to analyze the effects of materials and fabrication variables just in terms of the stresses and strains which are immediately affected, without totalling all the effects. In particular, for simple solenoidal magnetic fields generated by an unsupported coil, the calculated stresses and strains generated by the magnetic field are nearly independent of the material anisotropy, as discussed in section 5.8. We consider here only the stresses and strains related to fabrication and cooldown of the coil.

As the various stages of analysis were developed, the equations were programmed into our timeshare computer. The program as it exists on July 1, 1976 is listed in section 5.12. As will all such programs, developed by stages over a period of time, it is far from optimized in operation and format, but it does give useful results. The numerical results presented here were obtained with this program.

\subsubsection{Winding Tension}

First we consider the variation in internal stresses with different programs of winding tension. On an early model coil, the sponsor used a winding tension which decreased in four equally-spaced steps from the inside radius to the outside radius, as described in the following table (the coil fabrication data were presented in more detail in the previous report to the sponsor NBSIR-73-349):

\begin{tabular}{|c|c|c|c|}
\hline layer & $\begin{array}{l}\text { wire stress } \\
\left(\mathrm{N} / \mathrm{cm}^{2}\right)\end{array}$ & (assuming & $\begin{array}{l}\text { strain } \\
E=10.4 \times 10^{6} \mathrm{~N} / \mathrm{cm}^{2} \text { ) }\end{array}$ \\
\hline $1-10$ & 20500 & & 0.001973 \\
\hline $11-20$ & 15400 & & 0.001480 \\
\hline $21-30$ & 10300 & & 0.000986 \\
\hline $31-40$ & 7400 & & 0.000493 \\
\hline
\end{tabular}

This stepwise programming is not easily handled by our analytic model, but it can be approximated by assuming a linear variation between the inner and the outer radius. In figures 5-8 and 5-9, the resulting circumferential stress and radial stress (curves A) after epoxy impregnation and bobbin removal are compared with that obtained by three other possible winding tension programs: curve (B) a constant winding tension equal to the 


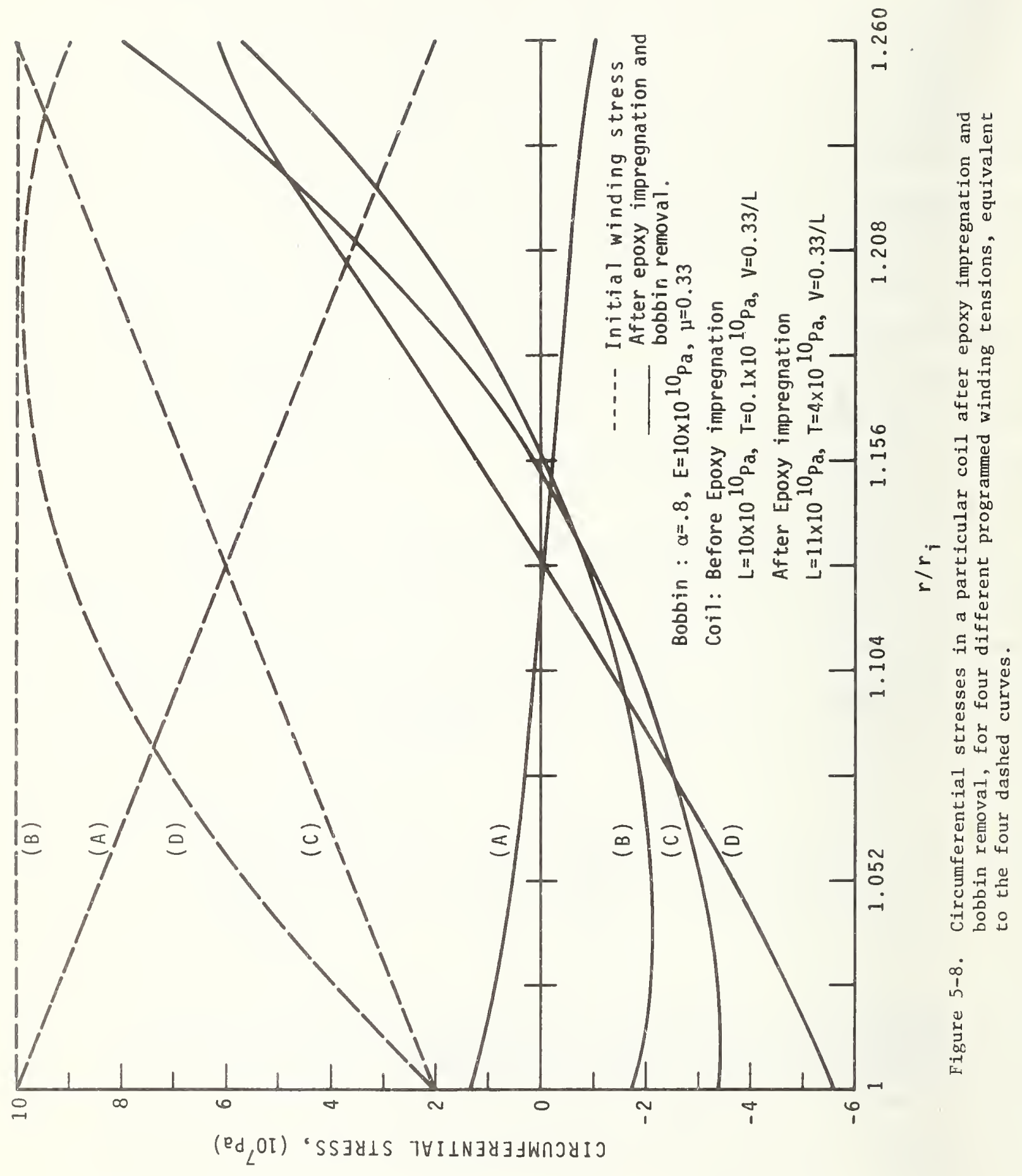




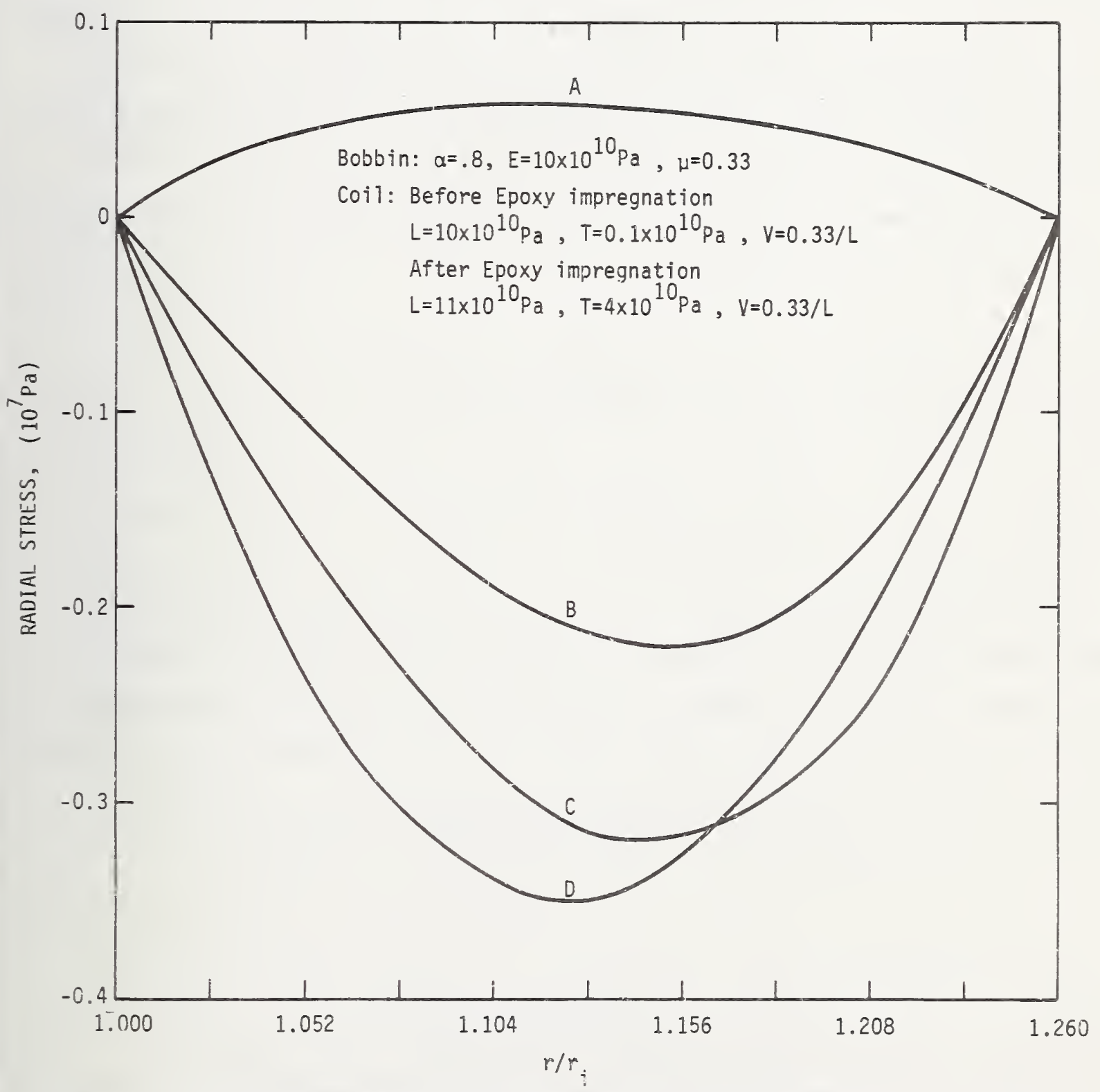

Figure 5-9. Radial stresses after epoxy impregnation and bobbin removal, for the four different programed winding tensions illustrated in Figure 5-8. Note the change from radial tension in curve A to radial compression in the other three curves. 
maximum tension of (A); curve (C), linearly increasing tension, with limits just reversed from that of curve (A); curve (D), a quadratically variable tension which rises rapidly from the lowest value used in (A) to the maximum, about $2 / 3$ of the way towards the outer radius, and then decreases. The dashed lines in the figures give a stress which is proportional to the programmed winding tension (actually it is $L \Sigma S_{i} r^{i}$, which equals the winding tensile stress times the area ratio, 0.73 ), and the solid lines give the resulting internal stresses after epoxy impregnation and bobbin removal, ignoring any thermal effects. The material and geometry parameters used in this calculation approximate those of the sponsor's model coil. Several important conclusions can be discussed with reference to these figures.

A decreasing winding tension, as in curve A, results in the lowest average circumferential stress, but also results in circumferential tension at the inner radius and radial tension throughout the coil. Radial tensile stress will tend to cause crack propagation at any bonding defect between the epoxy and the wire, and should thus be avoided. Furthermore, subsequent magnetic-field-induced stresses are higher at the inner radius than at the outer radius, so that a lower maximum stress under operating conditions will be obtained by creating a compressive circumferential stress at the inner radius during the fabrication and impregnation procedure.

Curves B, C, and D of figures 5-8 and 5-9 relate to different possible programmed winding tensions which were investigated in this study. All three have the advantage of providing both circumferential compression on the inner layers and radial compressive stress throughout the coil. Of these, the quadratic curve D provides the largest and most linear variation in circumferential stress from inside its outside radius, and thus will provide the lowest and most uniform stresses in the coil when the magnetic field is turned on.

It is important to note that the magnitude of the internal stresses (solid curves of figure 5-6) will scale linearly with the magnitude of the winding tension (dashed curves of figure 5-6). Hence, the higher the maximum tensile stress during fabrication, the higher will be the built-in internal strains before the coil is energized, and, assuming a programmed tension like C or D, of figure 5-8, the lower will be the maximum stresses when the coil is energized. The limit to this procedure for minimizing the operating 
stresses will be reached when the winding tension causes either yielding or a degradation in the superconducting properties of the wire. We conclude that this last topic needs careful attention.

\subsubsection{Effects of Properties Variations}

Numerical predictions, and the conclusions drawn from them, do depend on the assumed values of material properties, and on the coil configuration. It is important to investigate the sensitivity of the conclusions to changes in coil parameters.

Properties of the impregnated coil have been measured in our laboratory, but, for calculation of winding stresses, we must estimate the moduli of the un-impregnated coil.

The circumferential modulus, $E_{\theta}$, is that parallel to the wire, and can be estimated with reasonable accuracy knowing Young's modulus for the wire $\mathrm{E}_{\mathrm{w}}$ and the packing fraction, $\lambda$, of the wire in the winding. Since the glass cloth does rot provide much strength parallel to the wire,

$$
\mathrm{E}_{\theta} \approx \lambda \mathrm{E}_{w} .
$$

For the sponsor's coil

$$
E_{\theta} \approx 0.73 \times 10.4 \approx 7.6 \mathrm{MN} / \mathrm{cm}^{2}
$$

This is about the same as we have measured on the impregnated coil, which indicates that the epoxy does not add much strength parallel to the wire. The transverse modulus, $E_{r}$, is best estimated by considering the average modulus of a thickness d of glass cloth of modulus $E_{p}$ in series with a thickness $d_{w}$ of wire of modulus $E_{w}$, with the result

$$
E_{r}=\frac{E_{p} E_{W}\left(d_{p}+d_{W}\right)}{E_{p} d_{W}+E_{W} d_{p}} .
$$

Since $E_{w} \approx\left(10^{2}\right.$ to $\left.10^{3}\right) \times E_{p}$, this reduces to

$$
E_{I} \approx E_{P} \frac{d_{p}+d_{w}}{d_{P}}
$$

Our measurements on samples of the cloth show that its stress-strain behavior is quite hysteretic, represented approximately by

$$
\frac{\mathrm{d} \sigma}{\mathrm{d} \varepsilon} \approx 22 \sigma \quad \text { for increasing } \sigma
$$




$$
\frac{\mathrm{d} \sigma}{\mathrm{d} \varepsilon} \approx 50 \sigma \quad \text { for decreasing } \sigma
$$

for $\sigma$ in the range 5 to $5000 \mathrm{~N} / \mathrm{cm}^{2}$. To apply this result to our formalism based on linear or elastic material behavior, we take $d \sigma / d \varepsilon$ as a local modulus, valid for small variations around an average compressive stress within the coil. This is obviously not a good approximation, but to improve upon it within the framework of this general analysis would require very cumbersome non-linear stress-strain theory, and would not advance the project measurably.

The calculations discussed in 5.9.1 above, suggest that the magnitude of $\sigma_{r}$ for realistic values of the other parameters will be in the range from near-zero to about $2000 \mathrm{~N} / \mathrm{cm}^{2} ; 1000 \mathrm{~N} / \mathrm{cm}^{2}$ is a reasonable average, though it is difficult to know what relative weights should be given to the lower and higher ${ }^{-v a l u e s . ~ T h u s, ~ w i t h ~} d_{p} \simeq .004$ " and $\mathrm{d}_{\mathrm{W}} \simeq .021^{\prime \prime}$, we estimate

$$
\left(\mathrm{E}_{\mathrm{r}}\right)_{\mathrm{avg}} \approx 22 \times 1000 \times \frac{.025}{.004} \approx .15 \mathrm{MN} / \mathrm{cm}^{2}
$$

After epoxy impregnation, our measured value is

$$
\mathrm{E}_{\mathrm{r}} \approx 2.8 \mathrm{MN} / \mathrm{cm}^{2}
$$

Poisson's ratio in the transversely isotropic body is given by eqs (5-4) and (5-9). In this case with $E_{\theta}>E_{r}$, we take

$$
\left(\frac{\nu}{E}\right) \approx \frac{0.3}{E_{\theta}} \approx 0.04 \mathrm{~cm}^{2} / \mathrm{MN} \text {. }
$$

Figure 5-10 shows the effect of varying the radial compressive modulus over a realistic range encompassing the value which is estimated above. It is seen that the results are sensitive to the assumed value of $\mathrm{E}_{\mathrm{r}}$ before epoxy impregnation, but not so much so that the conclusions drawn in 5.7 above are significantly affected. Figures 5-11-13 compare calculated stresses with those obtained by Professor Young at Wisconsin, who was able to obtain a more accurate non-linear solution by iteration, using twodimensional finite-element program. (Professor Young's work was discussed earlier, in section 5.2). In these latter comparisons, a parabolic distribution of winding tension was assumed, approximately like curve D of figure 5-8. Figure 5-11 gives the circumferential stresses after winding but before epoxy impregnation. Figure 5-12 gives the 


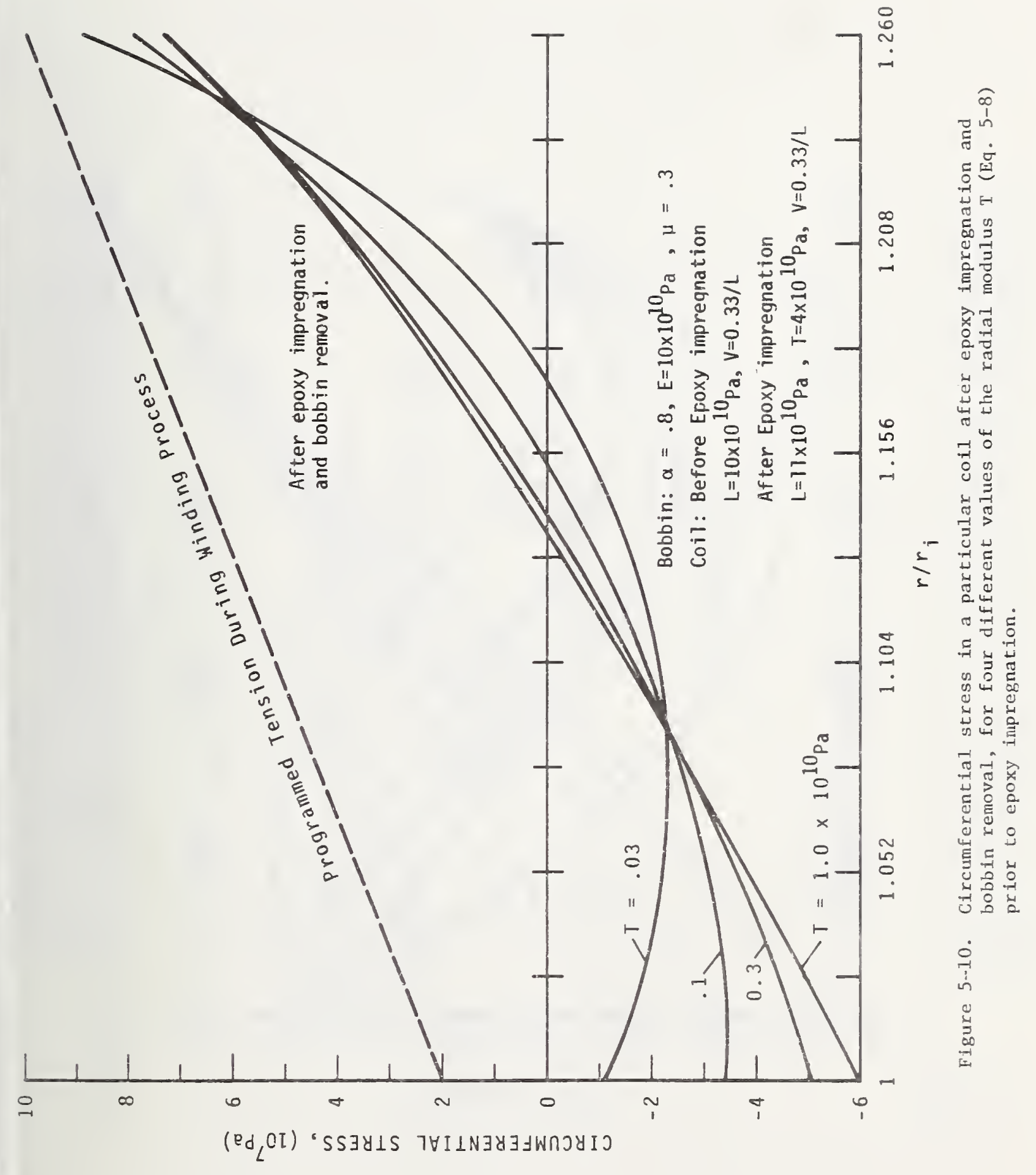




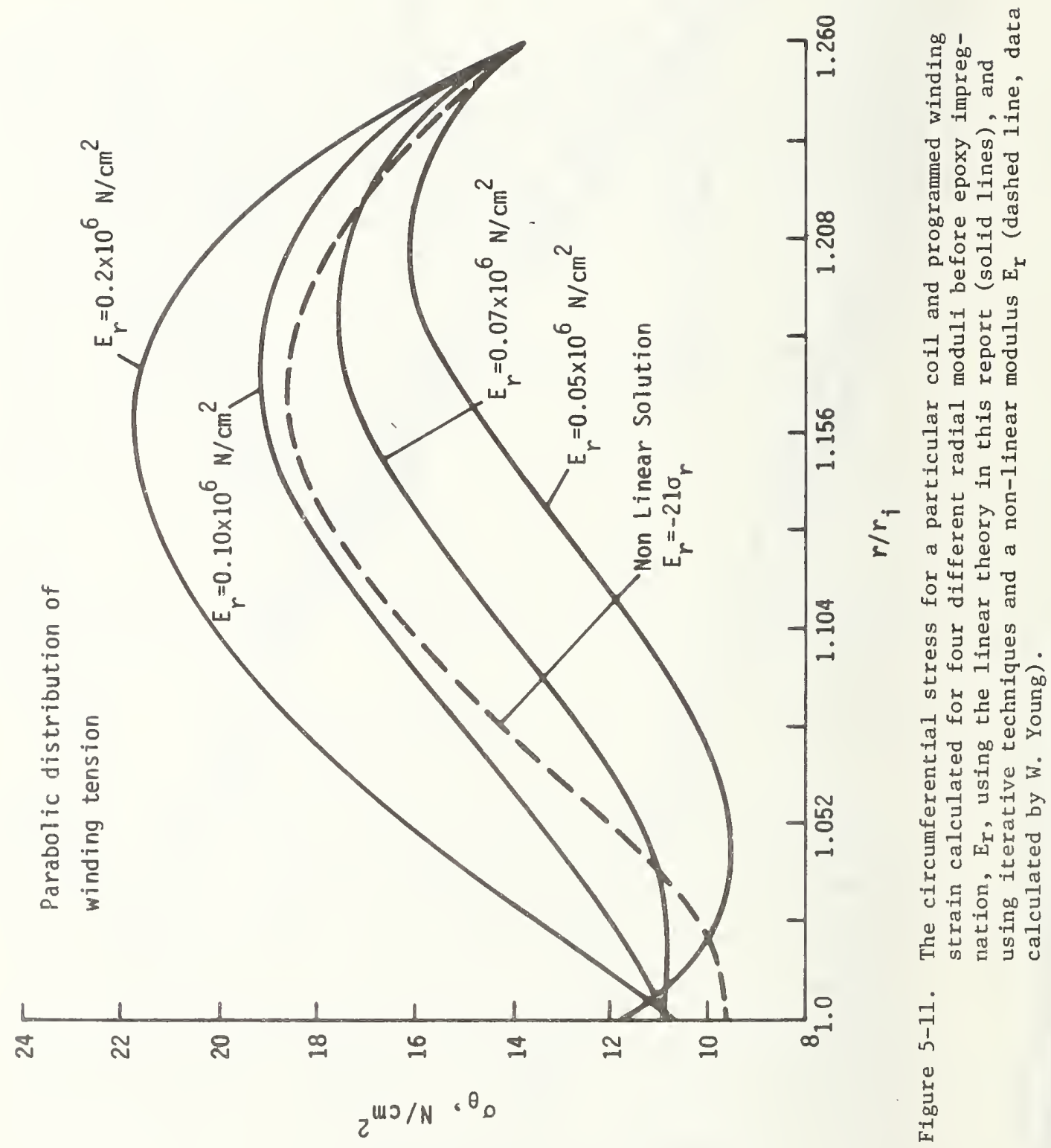




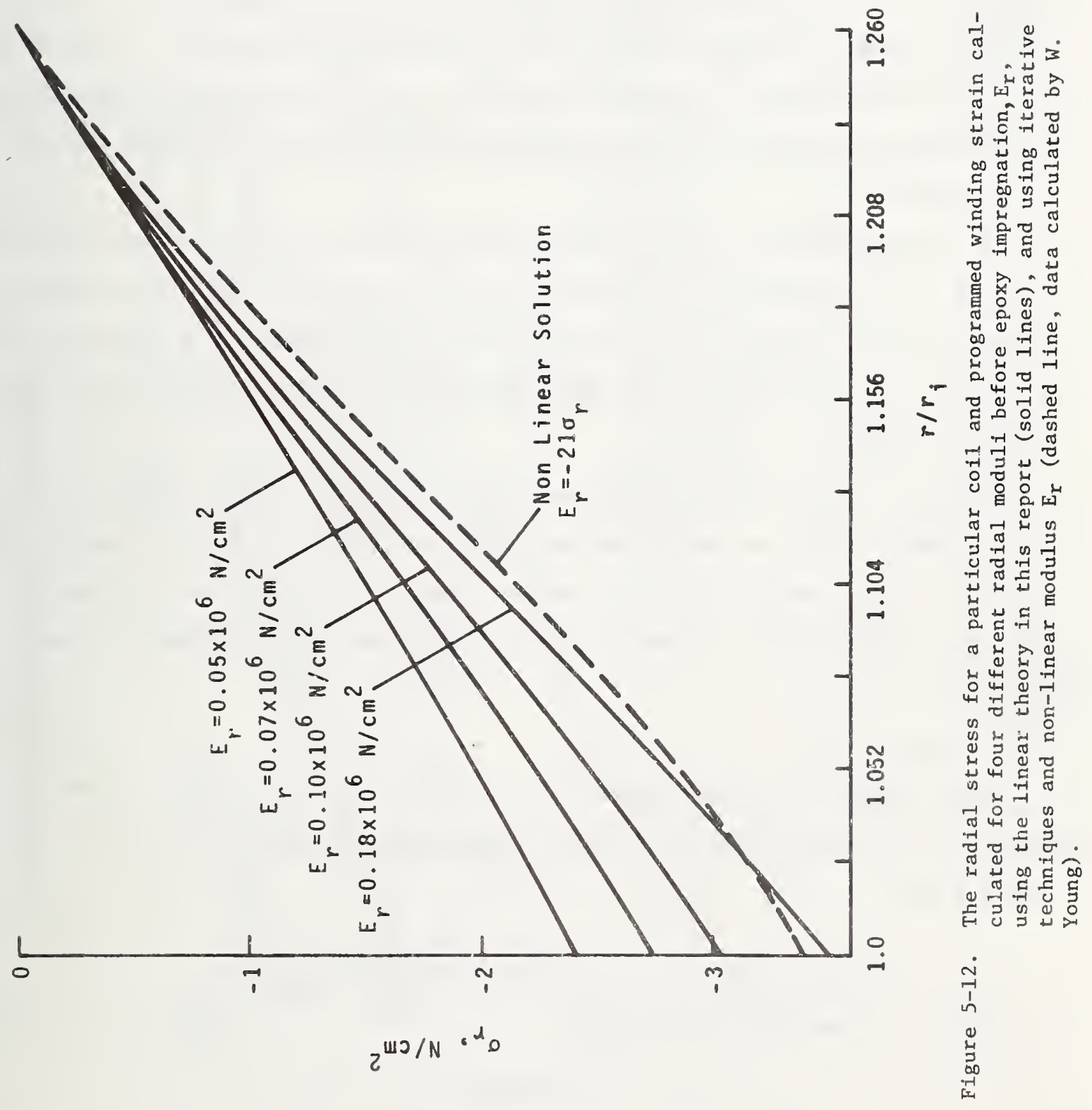


corresponding radial stresses. Figure 5-13 gives the change in circumferential stress following epoxy impregnation and bobbin removal. It is seen that the non-linear solution agrees reasonably well with the linear solution for $\mathrm{E}_{\theta} \approx 0.08 \mathrm{MN} / \mathrm{cm}^{2}$. This latter value is within the range which we would expect for an appropriate average value of $\partial \sigma / \partial \varepsilon$ over the cross-section, though it is lower than estimated above.

The variation in circumferential stress with bobbin characteristics is shown in figure 5-14. It is seen that the softer bobbin, i.e., as $\alpha \rightarrow 1$, gives a more uniform variation in stress with radius, and a larger total variation from inside to outside radius. Both of these are desirable. However, the variation is not very large and probably comparable with the uncertainty in the calculations due to the non-1inear glass cloth characteristics.

The circumferential stresses after epoxy impregnation and bobbin removal are plotted in figure 5-15 for coils of various thicknesses. It is seen that the thicker coils have a quite non-linear internal stress distribution, and considerable restraint must be used in extrapolating the results prescribed in this report for the sponsors test coil ( $g=1.26)$ to other geometries.

The sensitivity of the calculated stresses to changes In Poisson's ratio, eq 5-4, has also been tested. The results vary only about one percent as $(\nu / E)$ changes from 0 to $0.4 / \mathrm{E}_{\theta}$ for these two-dinensional calculations. However, we expect that the threedimensional stresses, from the magnetic field, will be reasonably influenced by the Poisson's ratio.

\subsubsection{Thermal Stresses}

Thermal stresses have been discussed in some generality in section 5.5. For the sponsors coil, our mechanical and thermal properties measurements on impregnated coil samples give

$$
\mathrm{L} \Delta \alpha \Delta \mathrm{T} \simeq-20900 \mathrm{~N} / \mathrm{cm}^{2}
$$

for contraction from $65^{\circ} \mathrm{C}$ to $4 \mathrm{~K}$ and

$$
\mathrm{k}=1.59 \text {. }
$$

With $g-1=0.26$, figures 5-2-4 give the results, that the circumferential stress at the inner radius will increase by 


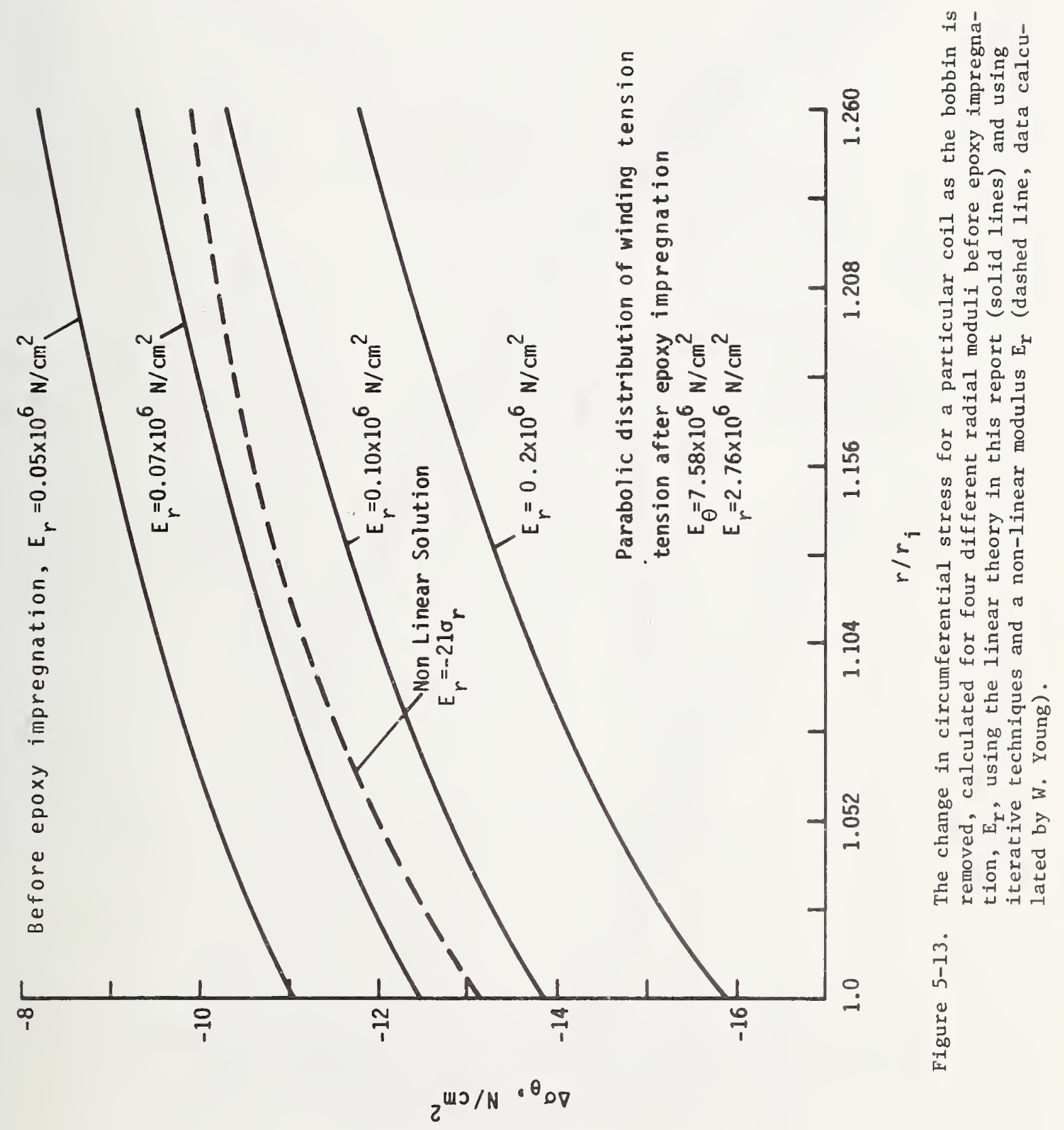




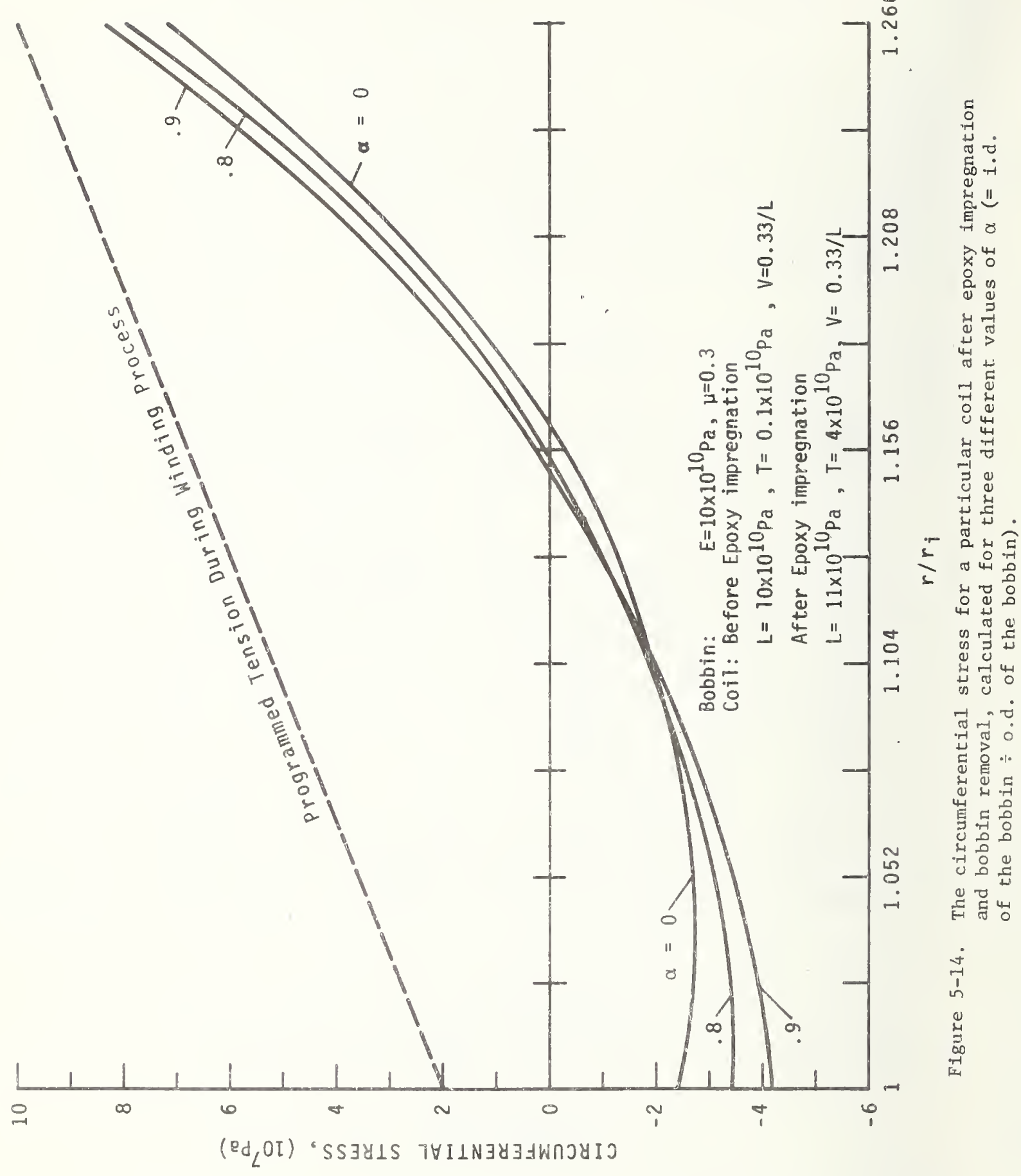




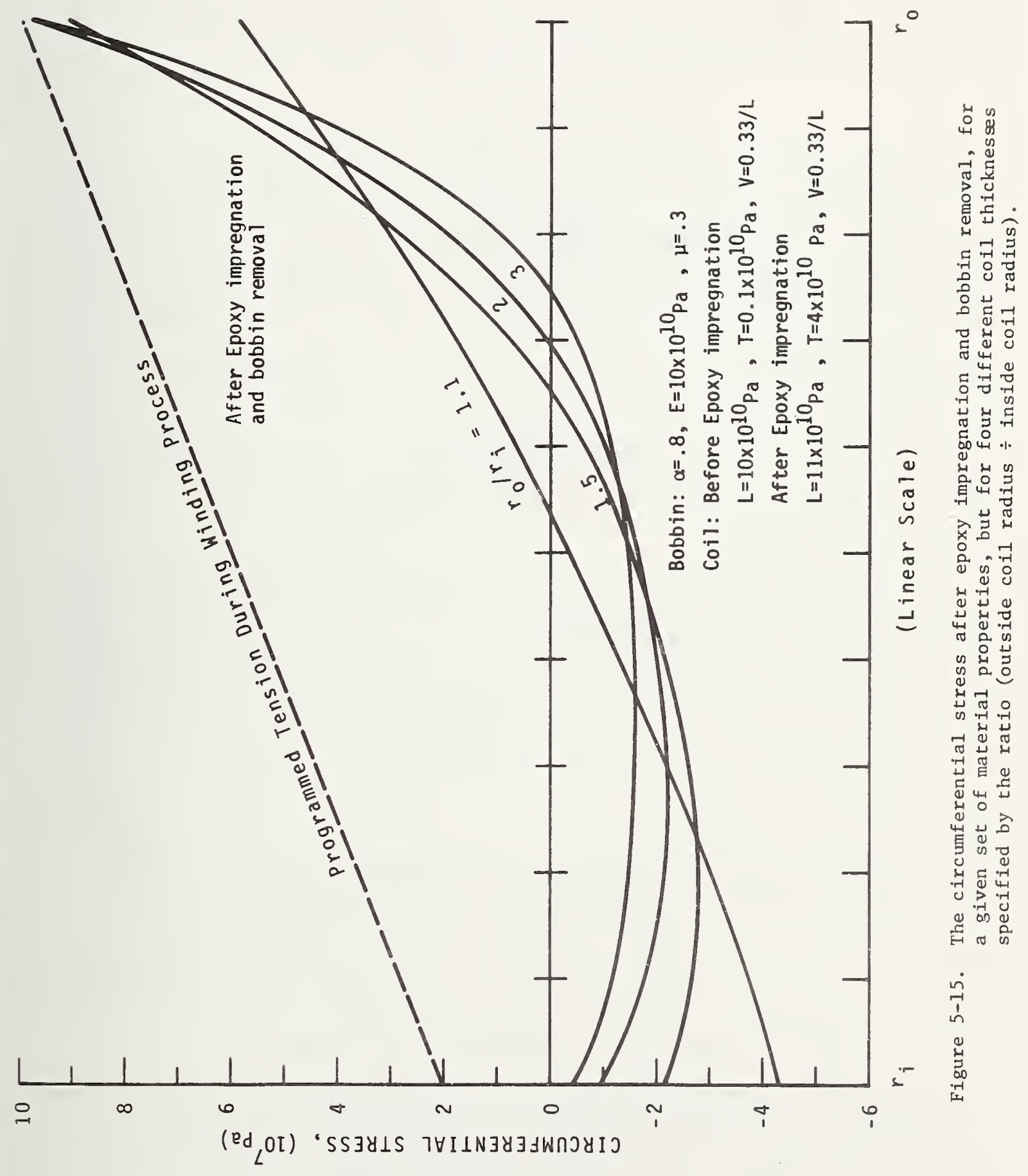




$$
\Delta \sigma_{c}\left(r_{i}\right) \approx 2700 \mathrm{~N} / \mathrm{cm}^{2}
$$

the circumferential stress at the outer radius will increase by

$$
\Delta \sigma_{C}\left(r_{0}\right) \approx-2000 \mathrm{~N} / \mathrm{cm}^{2}
$$

and the maximum radial stress will increase by

$$
\Delta \sigma_{\mathrm{r}} \approx+1300 \mathrm{~N} / \mathrm{cm}^{2}
$$

These are not large stresses, but unfortunately they all go the wrong direction, i.e., they tend to increase the maximum circumferential stress (at the inner radius) when the magnet is turned on, and to increase the opportunity for delamination due to radial tension. Considering the last point, it is important that the radial compression due to winding tension alwaýs be larger than the radial tension developed during cooldown, so that the cuculative radial stresses are always compressive, and delamination or crack propagation will be inhibited. This is another reason for using high winding tension during the fabrication process.

\subsubsection{Cumulative Stresses}

The cumulative circumferential and radial stresses during fabrication, cooldown, and magnetic field ope::ation are illustrated in figures 5-16 and 5-17. The solid curves in the figures refer to a model coil previously built by the sponsor. The dashed curves give predicted stresses for a coil of the same geometry, but fabricated differently: (a) wound on a thin-she11 aluminum bobbin rather than a solid stainiess steel one, (b) wound with a variable tension (curve "D") which increases from inside to outside radius about like curve D of figure 5-8, rather than decreasing from inside to outside radius, like curve A of figure 5-8. The dashed curves show a more uniform operating stress than do the solid curves, with a reduction of about $26 \%$ in the maximum circumferential stress and $44 \%$ in the maximum radial stress. These stress reductions are significant, and the potential of achieving them should be considered in future designs.

\subsection{Conclusions and Recommendations}

The analysis developed here provides a reasonable means for relating anisotropic materials and fabrication parameters to stresses and strains in transversely isotropic coil structures. Results are quite sensitive to both elastic modulus and thermal expansion anisotropy, especially as coil thickness is increased. When applied to the design 


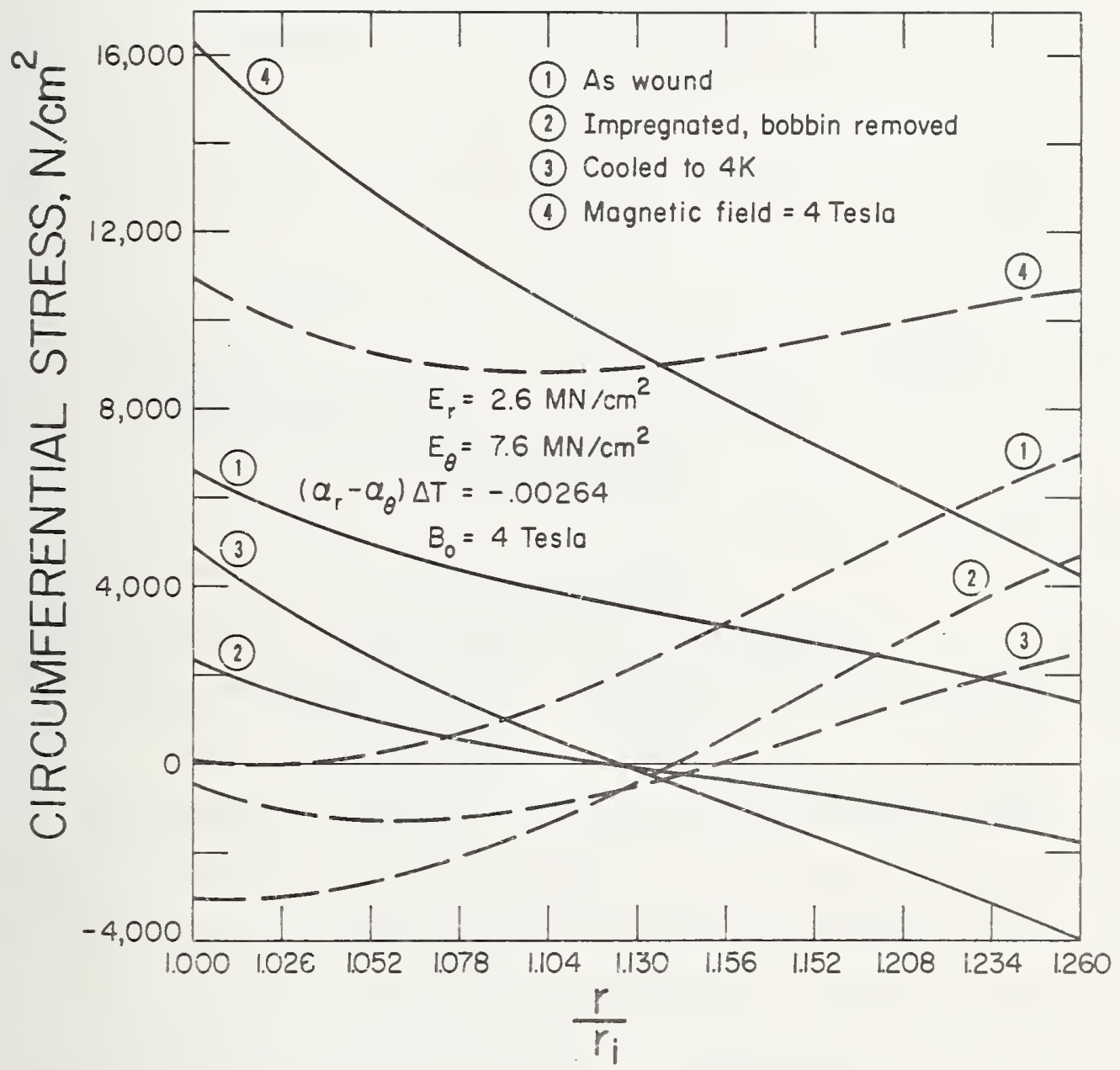

Figure 5-16. Cumulative clrcumferential stresses for a particular coll, calculated for a winding tension which decreases Iinearly from inside to outside radius (solid lines) and for a winding tension which increases parabolically from the minimum at the inner radius to a maximum in mid-winding and then decreases slightly (dashed lines). The maximum and minimum winding strains are the same in both cases. The dashed line (4) exhibits a significant reduction in maximum operating stress compared to the solid line (4). 


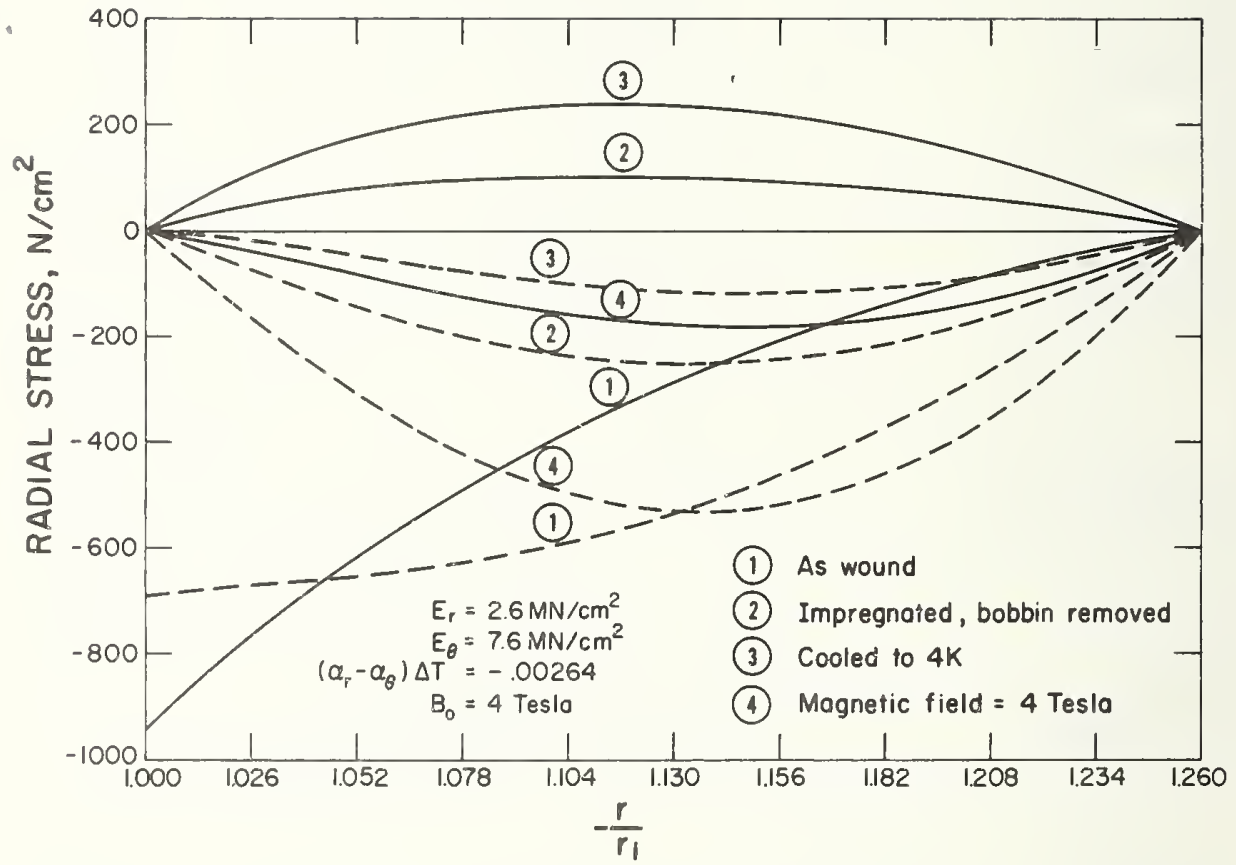

Figure 5-17. Cumulative radial stresses for a particular coil, calculated for a winding tension which decreases linearly from inside to outside radius (solid lines) and for a winding tension which increases parabolically from the minimum at the inner radius to a maximum in mid-winding and then decreases slightly (dashed lines). The maximum and minimum winding strains are the same in both cases. The dashed line (4) exhibits a significant reduction in maximum operating stress compared to the solid line (4). 
of a test coil for the sponsor, we can develop the following recommendations:

(1) The tension on the wire during the fabrication process should be programmed approximately as curve (D) in figure 5-8. On a practical basis it would probably have to be stepped from layer to layer.

(2) The maximum winding tension, e.g., the peak in curve $D$, should be as high as possible without causing subsequent degradation of superconducting wire performance. What this limit is should be determined.

(3) Peak operating stresses will be reduced if the composite radial modulus during the winding process were higher. This could be achieved by using a thinner cloth, or perhaps preimpregnating the wire and cloth and partially curing as winding progresses.

(4) Shrinkage of the epoxy during the curing process needs to be determined

(5) Peak operating stress will be lowered as the bobbin is made "softer". This could be accomplished by using a thin aluminum rather than a solid stainless steel mandrel. An equivalent effect could be obtained by wrapping the mandrel with several layers of glass paper before starting the winding.

Future work which should be pursued includes the following:

(a) The analysis should be enlarged to accomodate the effect of an overwrap or other mechanical constraint at the outer radius.

(b) Shrinkage stress during curing needs to be evaluated.

(c) The maximum allowable tensile stress during the winding process should be evaluated.

(d) Microscopic analysis needs to be done to evaluate the stress distribution from one wire through the epoxy to the next wire as a function of the average stress and component properties. This should lead to prediction of inter-laminar shear and, perhaps, to additional recomnendations on materials and fabrication techniques which are beyond the scope of this macroscopic analysis.

Note added in proof: Recent work discloses that the method developed here for calculation of winding stresses, Eqs. (5-28) to (5-41), is an approximation to the (exact) integration method mentioned on page 94. The results as outlined in this report agree within a percent or so with the exact calculations when the bobbin is quite stiff, i.e. when the bobbin modulus, B, defined by Eq. (5-37), is comparable with the winding modulus, L, Eq. (5-7). For softer bobbins (lower values of $B$ ), the exact calculation predicts a load relaxation at the inner winding layer which is up to $25 \%$ greater than predicted here (load relaxation 
is illustrated in Figure 5-5, p. 98). The errors from using this approximate calculation rather than the exact integration technique are not very serious for design purposes, and our conclusions above remain valid. 


\subsection{Nomenclature}

$a, b, c, d, C, D$

B

E

g

h

H

$\mathrm{k}$ or $\mathrm{K}$

L

$\mathbf{S}$

$r$

T

$\mathbf{u}$

V

$\alpha$

$\triangle$

$\varepsilon$

$\sigma$

\section{Subscripts}

$\mathrm{r}, \theta, \mathrm{z}$

B

i

$i$ and $o$

t adjustable coefficients

defined by eq. 5-27; an elastic modulus of the bobbin

elastic modulus

outside coil radius $\div$ inside coil radius

magnetic field at inner layer $\div$ magnetic field at center (defined by equation 5-54)

a quantity proportional to the square of the magnetic field (defined by equation 5-53)

mechanical anisotropy parameter (defined by equation 5-17)

longitudinal elastic modulus (defined by equation $5-7$ )

a strain coefficient, defined by equation 5-28

radius $\div$ inside coil radius

transverse elastic modulus (defined by equation 5-8)

$=\delta r$, a small radial displacement $\div$ inside coil radius

Poisson's ratio $\div$ elastic modulus (defined by equation 5-9)

thermal expansivity or inside radius $\div$ outside radius of bobbin (eq. 5-27)

inverse square of a modulus (defined by equation 5-12)

strain

stress

cylindrical coordinates

refers to bobbin

integers $1,2,3 \ldots$

insice and outside diameter (or radius)

transverse to wire; the $\mathrm{r}$ or $\mathrm{z}$ directions 
1 REM GO TO 9900 FOR INDEX TO VARIOUS SUBSECTIONS

5 I $2=0$ ! NONZERO VALUE SURFRESSES PRINTOUT OF WINDING STRESSES

20 DIM R $(11,2)$ ! RADIAL STRESS

21 MAT R=ZER

22 DIM $Q(11,2)$ ! THETA STRESS

23 MAT $Q=Z E R$

24 DIM U $(11,2)$ ! RADIAL DISPLACEMENT

25 MAT $U=Z E R$

26 DIM X(11) ! RADIAL POSITIONS

$30 \operatorname{DIM} A(4,4)$

$32 \operatorname{DIM} B(4,4)$

34 DIM C (4) ! GENERAL CONSTANTS

36 DIM F(4) !

38 DIM W(4)

40 DIM S(4)

"

! WIRE STRAIN POLONOMIAL COEFFICIENTS

INPUT WINDING STRAINS

42 DIM D(6) : ALPHAS IN THE U EQUATION

44 DIM E $(5,2)$ ! MODULI

46 DIM Y $(5,2)$ ! ALPHA DELTA T*S

50 DATA $.07,5.0,2.9,7.8,3.8,8.4,4.15,8.8,7.1,7.1$

51 MAT READ E

52 MAT $E=(1.0 E+06) * E$

60 DATA $1,1,1,1,-.00470,-.00275,1,1,-.00255,-.00255$

61 MAT READ Y

$102 \mathrm{R} 1=10$

$104 R 2=12.6$

$106 R 3=R 2+(.250 * 2.54)$

$108 \mathrm{R} 0=8$

110 H8 $=1.32$ ! LENGTH/I.D.

112 HO $=5 * 0.897$ ! CENTRAL FIELD, TESLA

$114 \mathrm{H} 9=1 / 0.897$ ! PEAK FIELD/CENTRAL FIELD

$116 \mathrm{H} 7=-0.2$ ! OUTSIDE FIELD/CENTRAL FIELD

$118 \mathrm{M} 7=(\mathrm{H} 7-\mathrm{H} 9) /(\mathrm{R} 2 / \mathrm{R} 1-1)$

124 GOSUB 7000

130 FOR $J=1$ TO 11

$140 \times(J)=R 1+(R 2-R 1) *(J-1) / 10$

150 NEXT J

170 TYPE "COIL R1, R2", R1,R2

200 PAUSE

215 DATA $0.3,0.9,1.1,0.9$

220 MAT READ S

222 MAT $S=(.001) * S$

230 TYPE "EFFECTIVE BOBBIN MODULUS", BO

295 TYPE "WINDING STRAIN", $S(1), S(2), S(3), S(4)$

300 REM EVALUATE W*S

310 MAT $A=I D N$

$320 \quad C(1)=R 1$

$322 C(2)=R 1+0.3 *(R 2-R 1)$

$324 C(3)=R 1+0.7 *(R 2-R 1)$

$326 C(4)=R 2$

330 FOR $I=1$ TO 4

340 FOR J $=1$ TO 4

$342 A(I, J)=C(I)^{\wedge}(J-1)$

344 NEXT J

346 NEXT I

350 MAT $B=I N V(A)$

352 MAT $W=B$ W

$360 \mathrm{~N}=1$

365 GOSUB 8000

375 TYPE "COIL, ER, EL, $K=", E(N, 1), E(N, 2), K$

400 REM SET UP STRESS EQUATIONS

$405 \mathrm{D} 1=1+\mathrm{B} 0 * \mathrm{~K} 6$ 


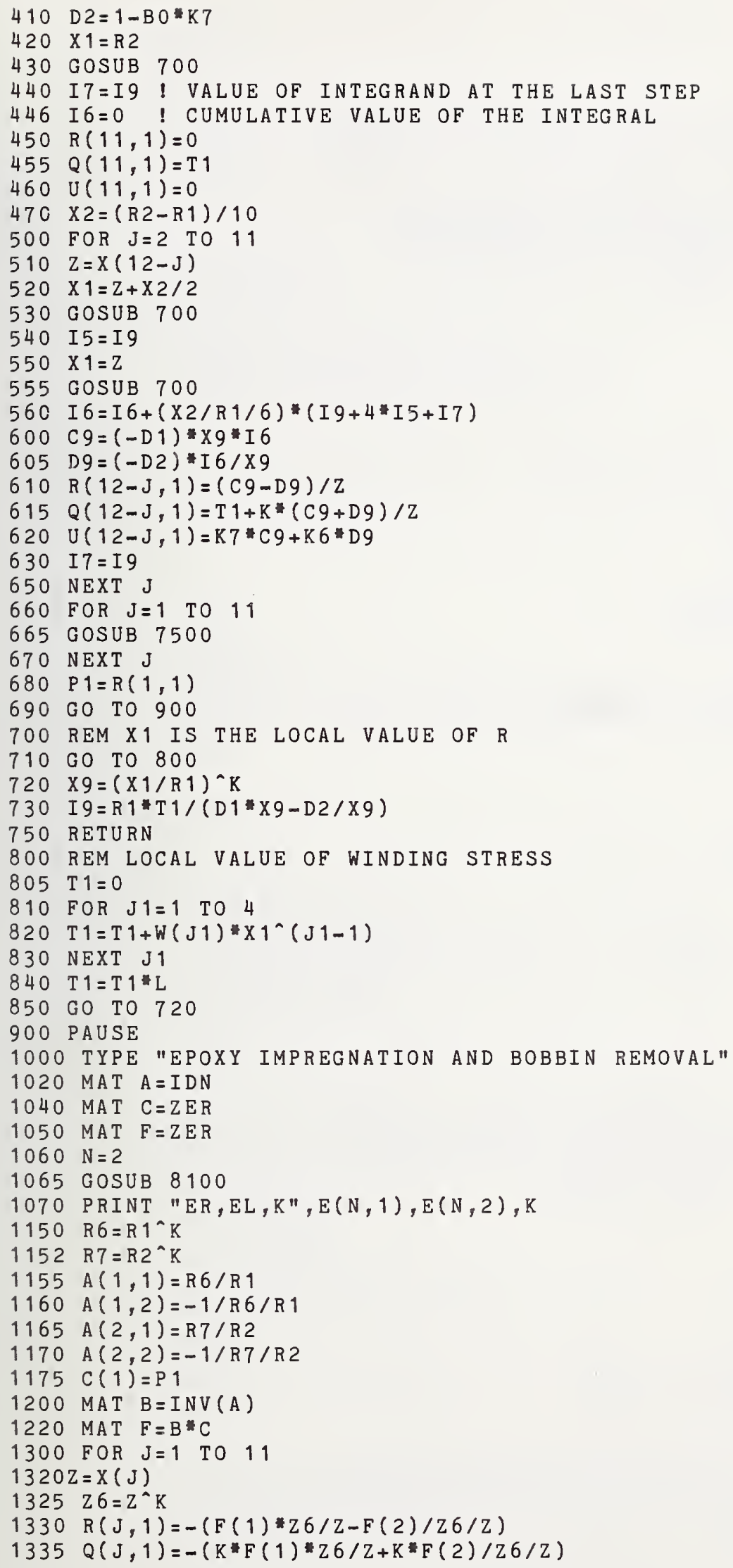




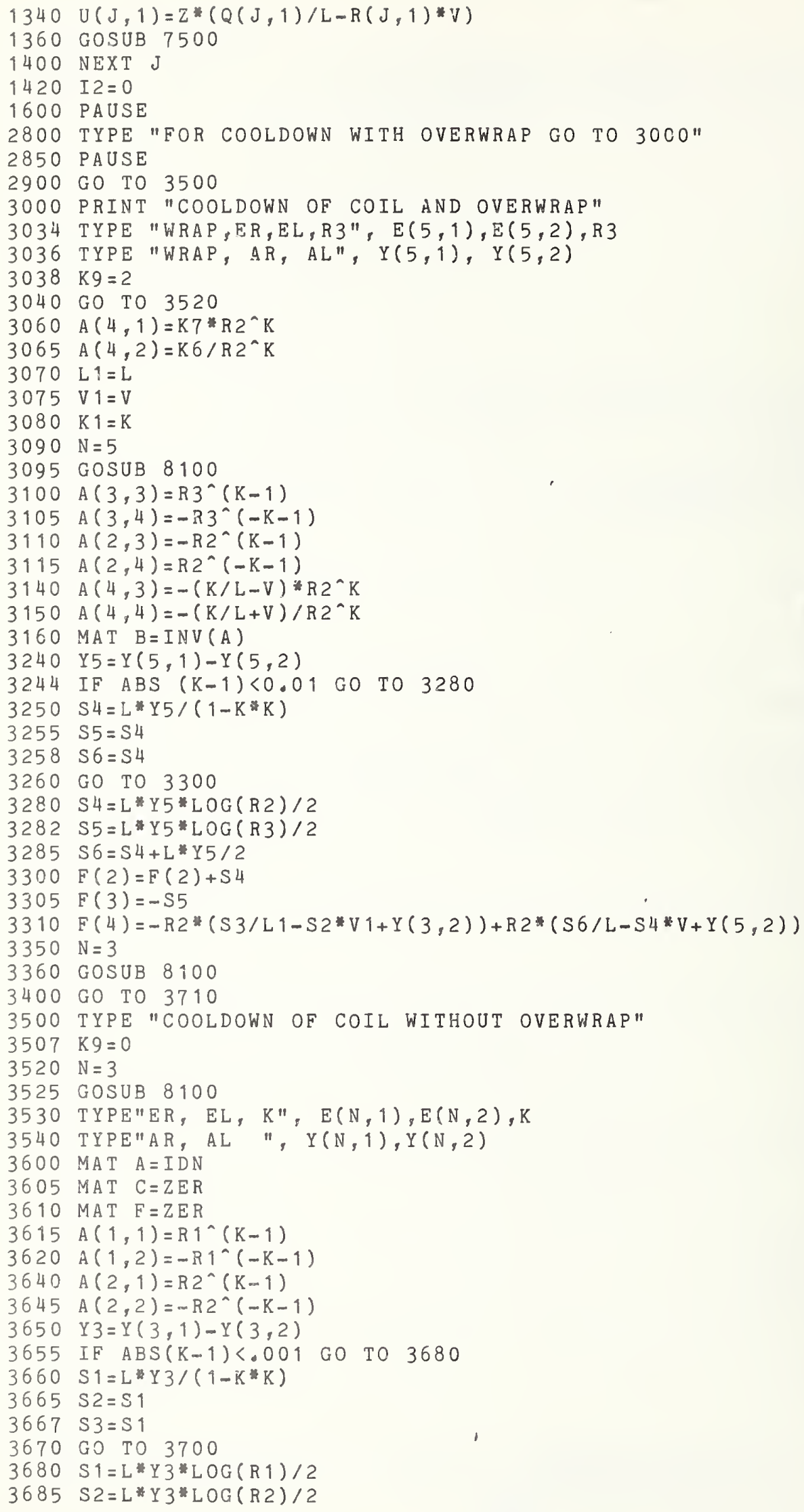




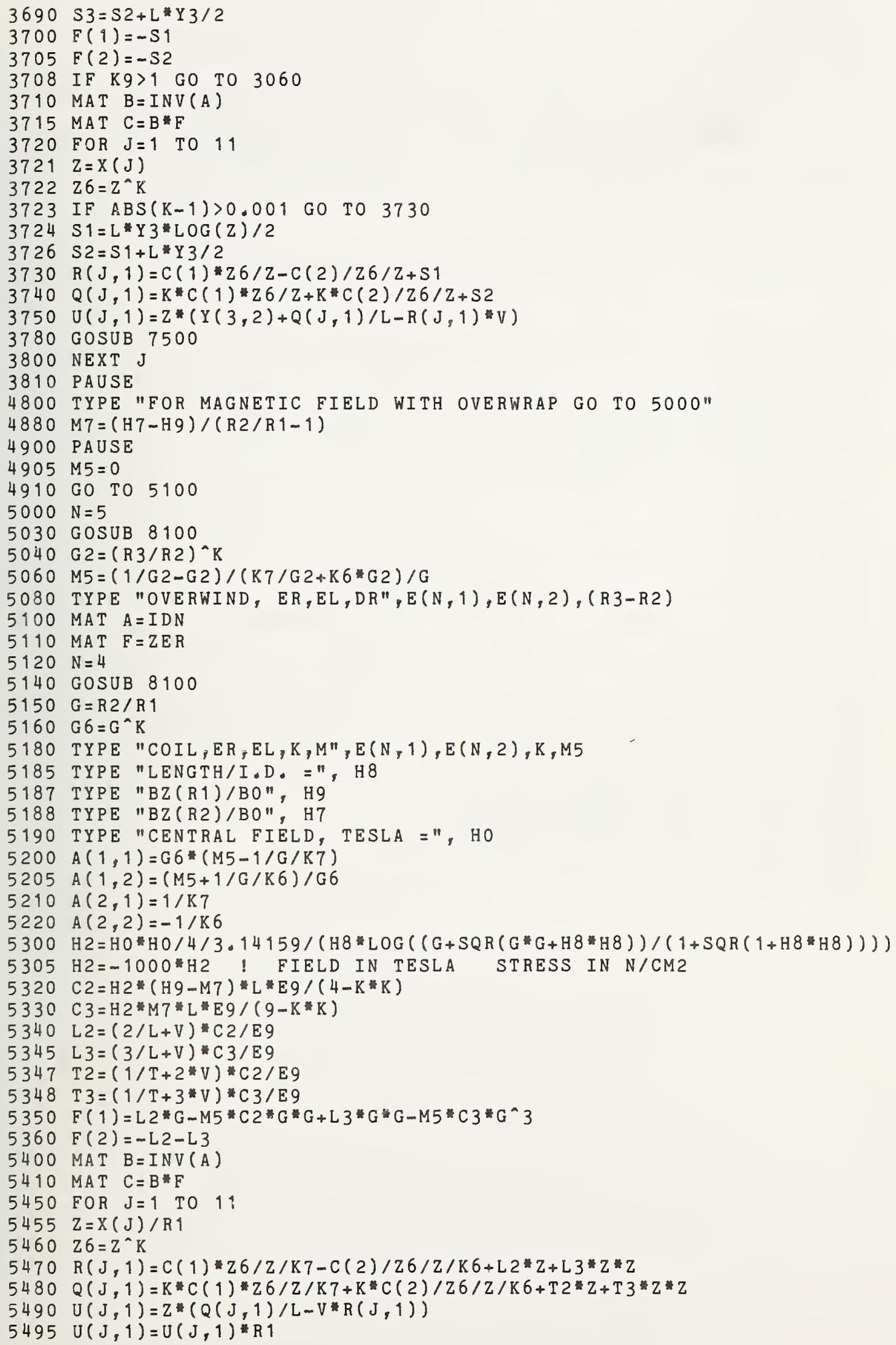




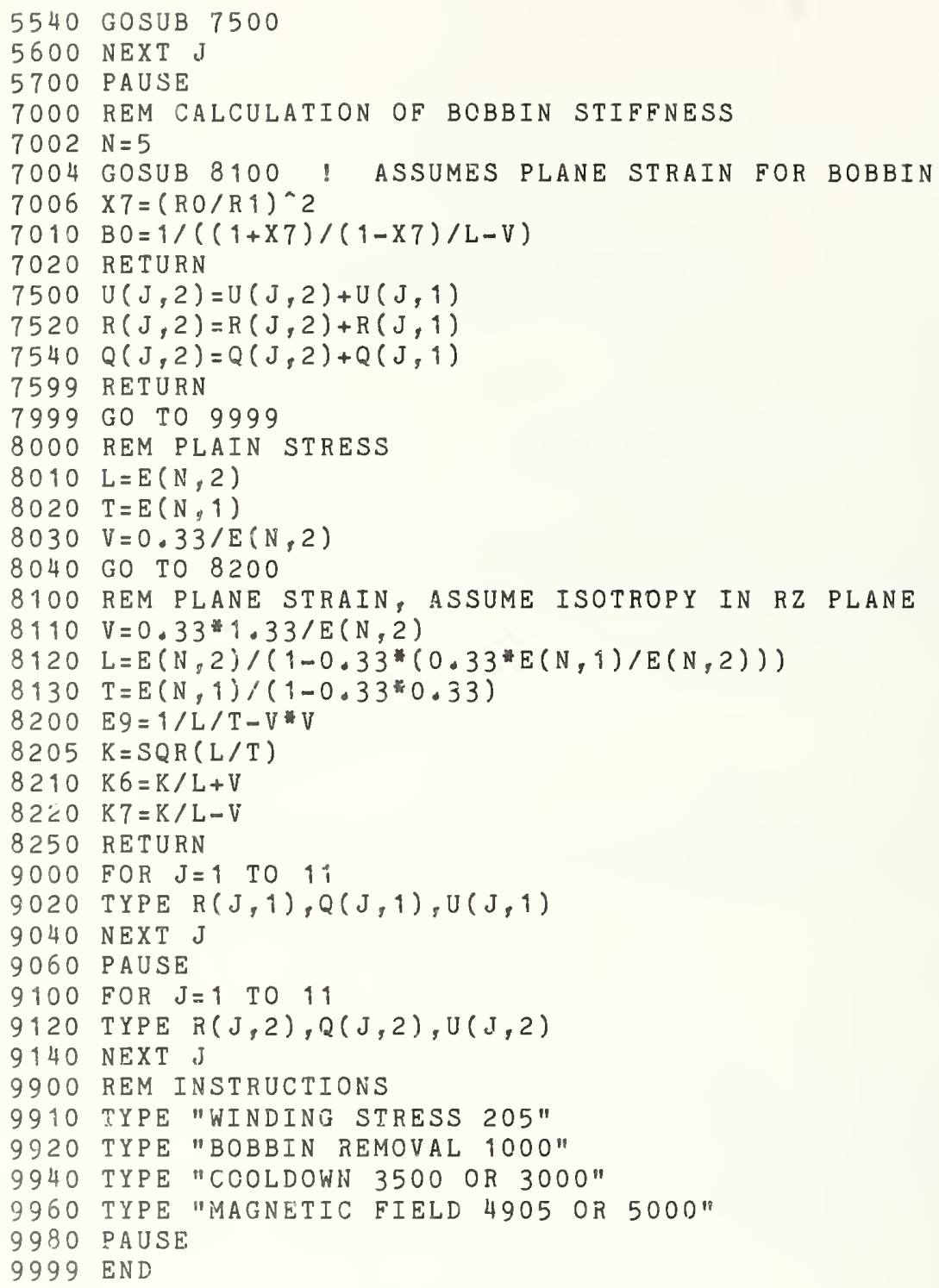


1. A. J. Middleton, P. D. Hey, and B. Colyer, "The Impregnation of Superconducting Windings with Epoxy Resins," Part I. Theoretical Considerations" (B. Colyer, only), and "Part II. Resin Data," Rutherford Laboratory Reports RHEL/R264 and RHEL/R265 (1972).

2. R. E. Sherrer, "Filament-Wound Cylinders with Axi-symmetric Loads," Journal of Composite Materials 1 , 345 (1967).

3. W. L. Fourney, "Residual Strain in Filament-Wound Rings," Journal of Composite Materials $\underline{2}, 408$ (1968).

4. B. R. Dewey and C. E. Knight, "Residual Strain in Layered Rings," Journal of Composite Materials $\underline{3}, 583$ (1969).

5. S. Timoshenko and J. N. Goodier, "Theory of Elasticity," Second Edition, McGraw-Hill Book Co. (1951).

6. J. Hord, "Stress Analysis of Tape-Wound Magnet Coils," Journal of Res. of NBS $\underline{69 C}$, 287 (1965).

7. J. Case, The Strength of Materials, Arnold and Company, London (1938). 


\section{DISCUSSION}

\subsection{Ccmparison with Predicted Values}

With a more complete test matrix we can now make further comparisons with the predicted values for the coil properties from the first report. ${ }^{1}$ Outlined in Table 6-1 are the calculated property values and the corresponding measured values at room temperature and $4 \mathrm{~K}$ for the $\mathrm{NbTi} / \mathrm{Cu}$-fiberglass epoxy superconducting coil composite. Generally, the calculated values are quite close to those measured and in several cases within a few percent. Calculation of property values at $4 \mathrm{~K}$ are not included because many of the constituent properties at $4 \mathrm{~K}$ are not avallable. Discussed below are some of the newly reported measurements.

The Young's modulii for the 1 and 2 directions were previously discussed and the calculated $\mathrm{E}_{33}$ is seen to be about $35 \%$ lower than the measured value. For the calculation the equation

$$
E_{c}=\frac{E_{f} E_{m}}{E_{f} V_{m}+E_{m} V_{f}}
$$

was used, where the subscripts $c, f$, and $m$ denote the composite, the wires, and the fiberglass epoxy matrix, respectively. $V_{f}$ is the volume fraction of the wires which in the 3 direction is $0.803 .^{1}$ As with $E_{22}$, this discrepancy is probably due to the very complex stress distribution in the epoxy around the wires and the assumption that the modulus of the matrix is that of the epoxy without fiberglass. Both of these would tend to raise the calculated values. A more sophisticated calculation ${ }^{1,2}$ gives a value of 3.16 which is now too high by $25 \%$. The calculations do, however, bracket the experımental value.

The measured value of the Folsson's ratio, $\nu_{32}$, is about $17 \%$ lower than that calculated using a complex equation ${ }^{2}$ assuming near isotropy in the 2 and 3 directions. This discrepancy probably reflects the small anisotropy in these two directions.

None of the shear modulii were previously reported and the agreement of $\mathrm{G}_{13}$ with the calculated value is quite good (less than $6 \%$ difference). The dynamic value (measured acoustically) may be more realistic since the static measurement was on a tightly wound coil ( $1.25 \mathrm{~cm}$ O.D). That the experimentally averaged value of $G_{21,31}$ is different from $G_{13}$ probably reflects again the slight anisotropy in the transverse directions, although it is quite close to the dynamic measurement. 
The shear modulus in the plane of isotropy, $G_{23}$ is calculated from

$$
G_{23}=E_{33} / 2\left(1+v_{23}\right)
$$

and depending upon the choice of the calculated $E_{33}$ from above either is much too low or agrees quite well. We are now compounding calculations and agreement must be considered fortuitous.

The thermal conductivity calculations are discussed in detail in Section 3.1 and the agreement is very satisfactory considering the approximations necessary for values for the fiberglass epoxy. The thermal expansion was discussed earlier and no calculations were done for the specific heat.

\subsection{Preliminary Coil Values}

Most of the experimental values have been obtained and we can generate a better set of constitutive relationships for the superconducting coil composite than those reported earlier. ${ }^{1}$ These should still be considered preliminary but would probably create much less error than any model used for their application. The experimental values used are those in Table 6-1 and the relationships are the same as in the first report. The few remaining calculated values are noted by an asterisk. Values at $4 \mathbb{K}$ are in parentheses just below the room temperature values.

The compliance matrix (for a transversely isotropic, orthotropic material) is in units of $10^{-10} \mathrm{~N}^{-1} \mathrm{~m}^{2}$;

$$
\begin{aligned}
& \begin{array}{llllll}
0.132 & -0.065 & -0.065 & 0 & 0 & 0
\end{array} \\
& (0.117)(-0.055) \quad(-0.055) \\
& \begin{array}{lllll}
0.364 & -0.101 & 0 & 0 & 0
\end{array} \\
& (0.234) \quad(-0.0781) \\
& \left.\begin{array}{cccc}
s_{i j}(295 K) \\
\left(s_{i j}\right)(4 R)
\end{array}\right) \quad \begin{array}{ccc}
(0.243) \\
0.929 & 0 & 0 \\
(0.643) & &
\end{array} \\
& 0.36400 \quad 0 \quad 0 \\
& \text { SYMETRIC } \\
& 1.310 \\
& (0.901)
\end{aligned}
$$


Table 6-1. Comparison of Calculated and Measured Values of Coll Composite Properties

\begin{tabular}{|c|c|c|c|}
\hline & \multicolumn{2}{|l|}{$293 \mathrm{~K}$} & \multirow{2}{*}{$\frac{4 \mathrm{~K}}{\text { Measurec }}$} \\
\hline & Calculated & Measured & \\
\hline$E_{11}\left(10^{10} \mathrm{~N} / \mathrm{m}^{2}\right)$ & \multicolumn{2}{|c|}{$\begin{array}{ll}\text { Young's Modulus } \\
6.66 & 7.55\end{array}$} & 8.52 \\
\hline $\mathrm{E}_{22}\left(10^{10} \mathrm{~N} / \mathrm{m}^{2}\right)$ & 1.96 & 2.75 & 4.11 \\
\hline $\mathrm{E}_{33}\left(10^{10} \mathrm{~N} / \mathrm{m}^{2}\right)$ & $\begin{array}{c}1.45 \\
(3.16)\end{array}$ & 2.28 & 3.10 \\
\hline$v_{13}$ & \multicolumn{2}{|c|}{$\begin{array}{ll}\text { Poisson's Rat1o } \\
0.345\end{array}$} & 0.352 \\
\hline$v_{21}$ & 0.11 & 0.172 & 0.226 \\
\hline$v_{32}$ & 0.335 & 0.278 & 0.321 \\
\hline & \multicolumn{2}{|l|}{ Shear Modulus } & \\
\hline $\mathrm{G}_{13}\left(10^{10} \mathrm{~N} / \mathrm{m}^{2}\right)$ & \multicolumn{2}{|c|}{$\begin{array}{l}0.67 \text { (STATIC) } \\
0.76 \text { (DYNAMIC) }\end{array}$} & 1.11 \\
\hline $\mathrm{G}_{21,31}\left(10^{10} \mathrm{~N} / \mathrm{m}^{2}\right)$ & 0.63 & 0.82 & 1.33 \\
\hline$G_{23}\left(10^{10} \mathrm{~N} / \mathrm{m}^{2}\right)$ & \multicolumn{2}{|c|}{$\begin{array}{cc}0.54 & 1.2 \\
(1.18) & \\
\end{array}$} & 1.58 \\
\hline$\lambda_{11}(\mathrm{~W} / \mathrm{mk})$ & \multicolumn{2}{|c|}{$\begin{array}{rr}\text { Thermal Conductivity } \\
208 & 169\end{array}$} & 169 \\
\hline$\lambda_{22}(\mathrm{~W} / \mathrm{mk})$ & 1.33 & 1.28 & 0.24 \\
\hline$\lambda_{33}(\mathrm{~W} / \mathrm{mk})$ & \multirow{2}{*}{\multicolumn{2}{|c|}{$\frac{4.3}{\text { Thermal Expansion }}$}} & -- \\
\hline & & & \\
\hline$\alpha_{11}\left(10^{-6_{k}^{-1}}\right)$ & \multicolumn{2}{|l|}{14.2} & 0.0 \\
\hline$\alpha_{22}\left(10^{-6} K^{-1}\right)$ & \multirow{2}{*}{\multicolumn{2}{|c|}{$\frac{21.2}{\text { Specific Heat }} 22.9$}} & 0.0 \\
\hline \multirow{2}{*}{$c_{p}\left(\mathrm{Jkg}^{-1} \mathrm{~K}^{-1}\right)$} & & & \\
\hline & -- & -- & 0.338 \\
\hline
\end{tabular}


where the $S_{i j}$ are used in the relation

$$
\varepsilon_{i j}=s_{i j} \sigma_{i j}
$$

between stress, $\sigma_{i j}$, and strain, $\varepsilon_{i j}$.

The thermal conductivity matrix simplifies to:

$$
\begin{aligned}
& 16900 \\
& \text { (169) } \\
& \lambda_{i j}(295 \mathrm{~K})\left(\mathrm{Wm}^{-1} \mathrm{~K}^{-1}\right)=0 \begin{array}{lll} 
& 1.28 & 0
\end{array} \\
& \left(\lambda_{i j}\right)(4 \mathrm{~K})\left(\mathrm{Wm}^{-1} \mathrm{~K}^{-1}\right)=\left(\begin{array}{ll}
0 & 1.28 \\
(0.24)
\end{array}\right. \\
& 0 \quad 0 \quad 4.3 * \\
& \text { (0.91*) }
\end{aligned}
$$

The thermal expansion matrix is as before:

$$
\begin{aligned}
& 14.0 \quad 0 \quad 0 \\
& \text { (6.8) } \\
& \alpha_{i j}(295 K)\left(K^{-1}\right)=\begin{array}{llll}
0 & 22.9 & 0
\end{array} \\
& \left(\alpha_{i j}\right)(77 K)\left(K^{-1}\right)=\quad \begin{array}{ll}
22.9 \\
(12.0)
\end{array} \\
& \begin{array}{lll}
0 & 0 & 22.9
\end{array}
\end{aligned}
$$

and the expansion at $4 \mathrm{~K}$ is, of course, zero so the values at $77 \mathrm{~K}$ have been given in parentheses.

The thermal diffusivity can also be calculated now from:

$$
D_{i j}=\frac{\lambda_{i j}}{\rho c_{p}}
$$

with the $4 \mathrm{~K}$ value for the specific heat and a measured density, $\rho$, of $6.0 \mathrm{gm} / \mathrm{cm}^{3}$. Not to be confused with the thermal expansion we have used $D$ as the symbol for thermal diffusivity. Its matrix is:

$$
\begin{aligned}
& \text { (83.3) } 0 \quad 0 \\
& \begin{array}{cccc}
\left(D_{i j}\right)(4 K)\left(\mathrm{cm}^{2} \mathrm{~s}^{-1}\right)= & 0 & (1.18) & 0 \\
0 & 0 & (4.49 *)
\end{array}
\end{aligned}
$$


The superconducting coil which is made up of wires is obviously anisotropic. The elastic properties, generally, have an anisotropy of about $2.5: 1$, the thermal expansion about 1.5:1, and the thermal conductivity (and diffusivity) of about 700:1 at $4 \mathrm{~K}$. As a function of temperature from room temperature to $4 \mathrm{~K}$, the elastic modulii increase 10-40\%, the thermal expansion goes to zero, and the thermal conductivity (and diffusivity) decreases by a factor of 1 to 5, depending upon the direction. In al.1 cases the anisotropy increases with decreasing temperature, being largest at $4 \mathrm{~K}$.

Basically, there are no unexpected trends in the data so far generated. At a fixed temperature or under fixed conditions, all of the behavior can be accomodated and the response of the coil to small or slow changes in its environment are predictable. The anisotropy in thermal expansion and very high anisotropy in the thermal diffusivity, however, could create significant stresses during cooldown or any thermal transients. The propagation of the normal zone in the superconductor will also follow the heat conduction down the wire. With further refinement of these measurements and comparison to measured coil behavior, even these effects should be calculable. These steps are part of the overall program and will be addressed in later reports.

\section{REFERENCES}

1. Fowlkes, C. W., Angerhofer, P. E., Newton, R. N., and Clark, A. F., "Characterization of a Superconducting Coil Composite," NBSIR 73-349, National Bureau of Standards, Boulder, CO (1973).

2. Adams, D. F. and Doner, D. R., "Transverse Normal Loading of a Unidirectional Composite," J. Composite Materials 1, 152 (1967).

3. Advanced Composites Design Guide, Volume 11, Analysis, Air Force Materials Laboratory (AFML/LC), Wright-Patterson Air Force Base, Ohio 45433 (January 1973). 


\begin{tabular}{|c|c|c|}
\hline $\begin{array}{l}\text { U.S. DEPT. OF COMM. } \\
\text { BIBLIOGRAPHIC DATA } \\
\text { SHEET }\end{array}$ & $\begin{array}{l}\text { 1. PUBLIC.ATION OR REPORT NO. } \\
\text { NBSIR } 76-837\end{array}$ & $\begin{array}{l}\text { 2. Gov't Accession } \\
\text { No. }\end{array}$ \\
\hline
\end{tabular}

4. TITLE AND SUBTITLE

Characterization of a Superconducting Coil Composite and Its Components

\begin{tabular}{|l}
\hline 7. AUTHOR(S) A. F. CTark, W. F. Weston, \\
R. J. Trapani \\
\hline 9. PERFORMING ORGANIZATION NAME AND ADDRESS \\
NATIONAL BUREAU OF STANDARDS \\
DEPARTMENT OF COMMERCE \\
WASHINGTON, D.C. 20234
\end{tabular}

12. Sponsoring Organization Name and Complete Address. (Street, City, State, ZIP)

Annapol is Laboratory

Naval Ship Research \& Development Center

Annapolis, MD 21402

5. Publication Date

3. Recipient's Accession No.

July 1976

6. Performing Organization Code

275.03

8. Performing Oigan. Report No. 10. Project/Task/Work Unic No. 2750538

11. Contract/Grant No. 13. Type of Report \& Period
Covered

14. Sponsoring Agency Code 15. SUPPLEMENTARY NOTES

16. ABSTRACT (A 200-word or less factual summary of most significant information. If document includes a significant bibliography or literature survey, mention it here.)

The physical properties of a superconducting coil composite and its components are studied in order to accurately predict the coil behavior under a variety of operating conditions. This second interim report includes data on the Young's moduli, the Poisson's ratios, the shear moduli, and the thermal conductivity of the coil composite as well as the compressive modulus of the fiberglass cloth and the thermal expansion of the superconducting wire. In addition, the development of an acoustic technique for the determination of the elastic moduli of the coil composite and the stress analysis of transversely isotropic magnet coils are reported.

17. KEY WORDS (six to twelve entries; alphabetical order; capitalize only the first letter of the first key word unless a proper name; separated by semicolons)

Fiberglass cloth; mechanical properties; superconducting coil composite; superconducting wire; stress analysis; thermal properties.

\begin{tabular}{|c|c|c|}
\hline $\begin{array}{l}\text { 18. AVAILABILITY } \\
\square \text { For Official Distribution. Do Not Release to NTIS }\end{array}$ & $\begin{array}{l}\text { 19. SECURITY CLASS } \\
\text { (THIS REPURT) } \\
\text { UNCL ASSIFIED }\end{array}$ & $\begin{array}{l}\text { 21. NO. OF PAGES } \\
142\end{array}$ \\
\hline $\begin{array}{l}\text { [7 Order From Sup. of Doc., U.S. Government Printing Office } \\
\text { Washington, D.C. } 20402, \text { SDCat. No.C13 }\end{array}$ & $\begin{array}{l}\text { 20. SECURITY CLASS } \\
\text { (THIS PAGE) }\end{array}$ & 22. Price \\
\hline $\begin{array}{l}X \text { Order From National Technical Information Service (NTIS) } \\
\text { SpringfieId, Virginia } 22151\end{array}$ & UNCLASSIFIED & $\$ 6.00$ \\
\hline
\end{tabular}


